\title{
Materials roles for promoting angiogenesis in tissue regeneration
}

\author{
Jung-Hwan Lee ${ }^{a, b, c, d, f, 1}$, Prakash Parthiban ${ }^{a, 1}$, Guang-Zhen Jin ${ }^{\text {a,b,c,f, }}$ \\ Jonathan C. Knowles ${ }^{\mathrm{a}, \mathrm{c}, \mathrm{e}}$, Hae-Won Kim ${ }^{\mathrm{a}, \mathrm{b}, \mathrm{c}, \mathrm{d}, \mathrm{f}, *}$ \\ a Institute of Tissue Regeneration Engineering (ITREN), Dankook University, Cheonan 31116, Republic of Korea \\ ${ }^{\mathrm{b}}$ UCL Eastman-Korea Dental Medicine Innovation Centre, Dankook University, Cheonan 31116, Republic of Korea \\ ${ }^{\mathrm{c}}$ Department of Nanobiomedical Science \& BK21 NBM Global Research Center for Regenerative Medicine, Dankook University, Cheonan 31116, \\ Republic of Korea \\ ${ }^{\mathrm{d}}$ Department of Biomaterials Science, School of Dentistry, Dankook University, Cheonan 31116, Republic of Korea \\ e UCL Eastman Dental Institute, 256 Grays Inn Road, London WC1X 8LD, United Kingdom \\ ${ }^{\mathrm{f}}$ Cell \& Matter Institute, Dankook University, Cheonan 31116, Republic of Korea
}

\section{A R T I C L E I N F O}

\section{Keywords:}

Angiogenesis

Tissue regeneration

Materials properties

Delivery

Cell engineering

\begin{abstract}
A B S T R A C T
Enabling angiogenesis is critical for the success of tissue repair therapies and the fate of tissueengineered constructs. Although many biochemical signaling molecules have been used, their biological functions in vivo are known to be limited, mainly due to their short lifetime and poor activity. Matrices (or engineered biomaterials), beyond the biochemical signals, play pivotal roles in stimulating angiogenic processes. Here we discuss the proangiogenic effort taken to repair and regenerate various tissues including skin, bone, muscle and nerve, focusing on the roles of engineered matrices. This includes the design of pore structure and physico-chemical properties (nanotopology, stiffness, chemistry and degradability), the tailoring of matrices for proper presentation of growth factors and their crosstalks with adhesion ligands, the controlled and sustained delivery of angiogenic molecules and metallic ions, and the engineering of cells and construction of prevascularized tissues. Collectively, the materials-driven strategies are envisaged
\end{abstract}

\footnotetext{
Abbreviations: ALK, activin receptor-like kinase; $\mathrm{Ca}_{2} \mathrm{MgSi}_{2} \mathrm{O}_{7}$, akermanite; ALP, alkaline phosphatase; ANG/TIE, angiopoietin/tyrosine kinase

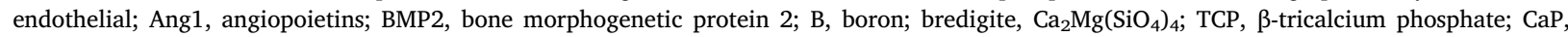
calcium phosphate; CPC, calcium phosphate cement; CNPs, cerium oxide nanoparticles; CAM, chicken chorioallantoic membrane; Co, cobalt; CMP, collagen-binding component; $\mathrm{Cu}$, copper; $\mathrm{Ca}_{2} \mathrm{SiO}_{4}$, dicalcium silicate; EC, endothelial cell; eNOS, endothelial nitric oxide synthase; EPCs, endothelial progenitor cells; HUVECs, enhanced human umbilical vein endothelial cells; EGF, epidermal growth factor; ECM, extracellular matrix; FGF, fibroblast growth factor; FCs, fibroblasts; FN, fibronectin; GelMA, gelatin methacrylate; GFs, growth factors; HBD, heparin binding domain; HGF, hepatocyte growth factor; HOPs, human osteoprogenitor cells; HA-MMP, hyaluronic acid with matrix metalloproteinase; HA, hydroxyapatite; HIF$1 \alpha$, hypoxic inducing factor; KDR, kinase insert domain receptor; LG, laminin-type G; Mg, magnesium; MMPs, matrix metallopeptidases; MSCs, mesenchymal stem cells; MBG, mesoporous bioactive glass; $\mathrm{Ca}_{2} \mathrm{SiO}_{3}$, monocalcium silicate; MDSCs, muscle-derived stem cells; NSL, nanosphere lithography; PDGF-BB, platelet-derived growth factor-BB; PEA, poly(ethyl acrylate); PEG, Poly(ethylene glycol); PLGA, poly(lactic-co-glycolic acid); PMA, poly(methyl acrylate); PMMA, poly(methyl methacrylate); PAAm, polyacrylamide; PCL, polycaprolactone; PDMS, polydimethylsiloxane; pGlcNAc, poly-N-acetyl glucosamine; PHD2, prolyl hydroxylase 2; ROS, reactive oxygen species; RANKL, receptor activator of nuclear factor-kappa B ligand; RUNX2, runt-related transcription factor 2; SBGs, silica-based bioactive glasses; $\mathrm{CaCO}_{3}$, silicate-doped calcium carbonate; SMCs, Smooth muscle cells; Laponite, sodium-lithium-magnesium-silicate; SVF, stromal vascular fraction; SDF1, stromal-derived factor 1; Sr, strontium; 3D, threedimensional; TEVGs, tissue engineered vascular grafts; Ti, titanium; TGF- $\beta 1$, transforming growth factor- $\beta 1 ; \mathrm{Ca}_{3} \mathrm{SiO}_{5}$, tricalcium silicate; VEGF, vascular endothelial growth factor; Zn, zinc

* Corresponding author at: Institute of Tissue Regeneration Engineering (ITREN), Dankook University, Cheonan 31116, Republic of Korea.

E-mail address: kimhw@dku.edu (H.-W. Kim).
}

${ }^{1}$ Both authors equally contributed to this work. 
to tune the cell and tissue microenvironments where angiogenic events can be significantly favored through the matrix cues and properly presented or delivered signaling molecules and cells.

\section{Introduction}

A fundamental issue in engineering damaged tissues including skin, nerve, muscle and bone is to create blood vessels which can provide nutrients and oxygen to the cells buried deep inside the tissue while removing waste away from the cells. The spatial distribution of the vessels inside the synthesized tissue plays an important role in the viability of the cells around it. If the cells are further than 100-200 $\mu \mathrm{m}$ from the nearest blood vessel, they die due to a lack of oxygen and nutrients [1,2]. Hence, any effort in designing angiogenic biomaterials and engineering the vasculature formation needs to take this crucial point into consideration [3-8] (as shown in Fig. 1).

Blood vessel formation and remodeling in vivo take place via the process of vasculogenesis, angiogenesis, and arteriogenesis [9]. Werner Risau, a pioneer in the field, defined the formation of blood vessels during embryogenesis as a two-step process [10]. The first step is the formation of angioblasts - a subset of primitive mesodermal cells committed to differentiate into endothelial cells. The second step is the formation of primitive blood vessels from the angioblasts at or near the site of their origin. This two-step process that leads to the onset of vascularization is termed vasculogenesis [11]. In adults, vasculogenesis takes place in tissue repair or tumor growth, where endothelial progenitor cells (EPCs) home to a damaged site and differentiate into endothelial cells to form primitive vascular networks [12].

Angiogenesis, on the other hand, is the formation of blood vessels in adults owing to a direct response to tissue demand, and predominately occurs from preexisting blood vessels, as illustrated in Fig. 2. During angiogenesis process, the vascular permeability increases, allowing the extravasation of plasma proteins, and the degradation of basement membrane with remodeled extracellular matrix (ECM) releases ECM-mediated growth factors while allowing pericyte-endothelial cell (EC) contacts. The ECs invade to the surrounding tissue and organize into multicellular tubes containing functional lumens $[13,14]$. In tissue repair processes like bone regeneration, angiogenesis is the most efficient and quickest way for an injured site to create neo-blood vessels to support the nutrients and oxygen for cellular functions.

Regeneration of tissues entails complex and dynamic processes where vascularization followed by angiogenesis is necessary to maintain homeostasis. For example, the complex interplay of auto- and paracrine cytokines/growth factors/exosomes produced by surrounding osteoblasts, endothelial cells and their precursors, and migrated mesenchymal stem cells and macrophage plays critical roles in dynamic process of bone defect regeneration/repair via angiogenesis [15-17]. In skeletal muscle repair/regeneration, increasing angiogenesis via vascular endothelial growth factor (VEGF) release can efficiently clear out damaged cell structures and their byproducts such as damaged associated molecular patterns and start to recruit stem cells/precursors for skeletal muscle repair/ regeneration deliver nutrients and signaling factors, reducing the development of mal-functioned traumatic fibrosis [18]. Along with above examples, angiogenesis basically supports delivery of nutrients and signaling factors and removal of waste products, thus promoting neo-tissue formation [15]. In this process, many signaling molecules and cytokines are produced to promote blood vessel formation and tissue regeneration. Also, different types of cells, including progenitor/stem cells, endothelial cells and tissue lineage cells mutually communicate in secreting the signaling molecules, ultimately to regenerate fully vascularized tissues. Therefore, exploring strategies to recapitulate the signaling molecular cascade and cellular events that are favorable for angiogenesis and vascularization is crucial for tissue regeneration.

Materials for biomedical applications aim to repair and regenerate damaged tissues [19]. For the successful tissue regeneration, materials should allow the vascularization to recruit cells and populate them, and further to stimulate the production of tissue matrix molecules. Given the importance of interplay between tissue repair and angiogenesis, it is essential to develop materials that promote the angiogenic events and ultimately lead to the formation of re-vascularized tissue.

Here, we systematically review the angiogenesis strategies based on materials to repair and regenerate damaged and diseased tissues including skin, bone, muscle and nerve. On the basis of extensive literature surveys, strategies are categorized into i) tailoring of materials with appropriate physico-chemical properties such as pore architecture, surface nano/micro-topology, physical stiffness,
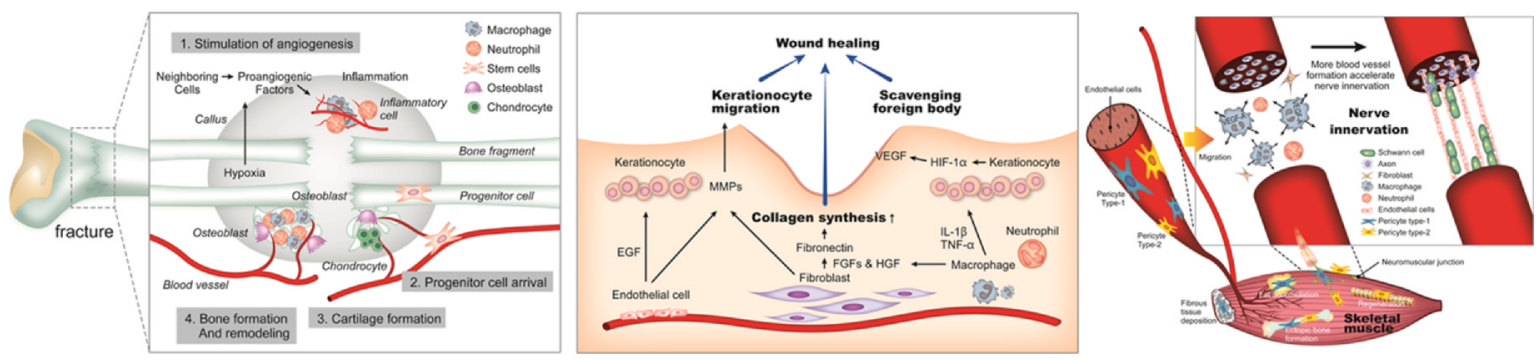

Fig. 1. Angiogenesis process essentially involved in the healing and repair of various damaged tissues. (a) Bone fracture repair, (b) skin wound healing, and (c) nerve innervation. 


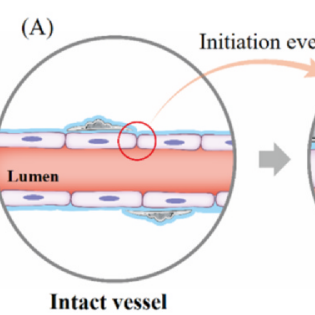

Intact vessel
(B)

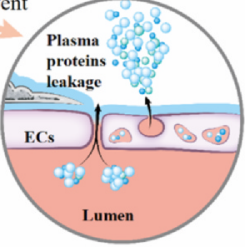

Vascular permeability

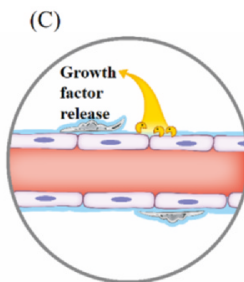

Degradation of ECM

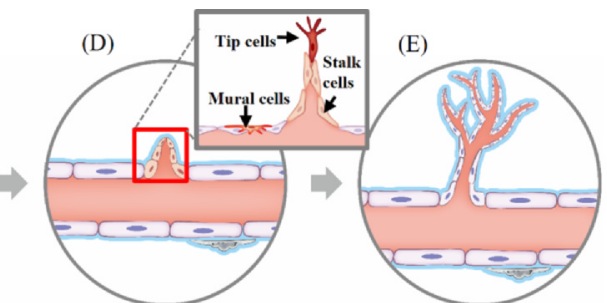

ECs migration/proliferation

Blood vessel morphogenesis

Fig. 2. Schematic showing the angiogenesis process. Stable vessels (A) undergo a vascular permeability increase, which allows extravasation of plasma proteins (B). Degradation of the ECM by matrix metallopeptidases (MMPs) relieves pericyte-EC contacts and liberates ECM-sequestered growth factors (C). ECs then migrate to their final destination and proliferate to assemble into tip and stalk cells (D), which further develops into many branches (E).

and chemistry, ii) design of matrices for proper presentation or crosstalks of growth factors, iii) modulation of scaffolds for controlled or sustained delivery of signaling molecules (i.e., angiogenic factors, metallic ions), and iv) engineering of scaffolds for cell culture and delivery. This Review is envisaged to give some insightful information on the angiogenesis events related with materials, and to guide researchers to design and develop materials for successful tissue regeneration through promoted angiogenesis.

\section{Tailoring of pore structure and physico-chemical properties}

Scaffolds with a three-dimensional (3D) open-pore structure are the most common form of biomaterials used to repair tissues. The pore space initially empty is quickly filled with biological molecules and cells, and a series of vascularization events occur to help the repair process. To enhance and accelerate the blood vessel formation, considerable effort has been directed to optimize the 3D scaffolds in terms of pore structure (e.g., pore size, porosity, and interconnectivity) and physico-chemical properties (e.g., surface chemistry, surface topography, degradability, and stiffness) [20-27].
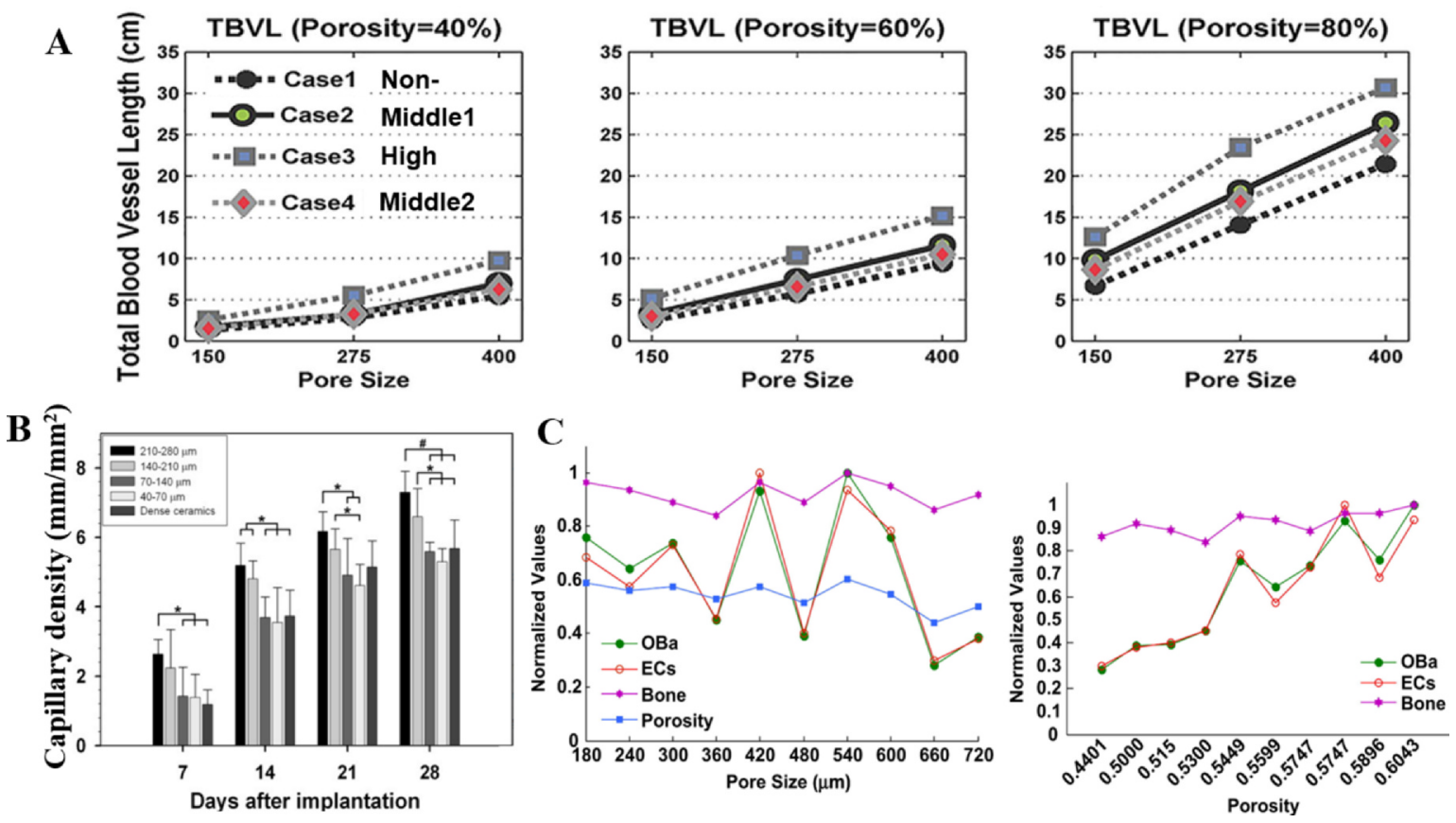

Fig. 3. Pore structure of scaffolds determines vascularization. (A) Among others, higher porosity, larger mean pore size, and rapid degradation were found to be important factors to increase vascularization in porous scaffolds, revealed by computer modelling studies [29]. In addition, the multilayered scaffold improved vascularization compared to the single-layer one. (B) Calcium phosphate (CaP), being a typical bone bioceramic, was prepared into particles with varying pore sizes (40-70, 70-140, 140-210, and 210-280 $\mu \mathrm{m}$ ) and the ability to induce angiogenesis, vascularization and osseointegration was examined in critical-sized cranial defects in-vivo model [32]. Increase of pore sizes accelerated capillary encapsulation in scaffold. (C) The correlations between the scaffold porosity and the number of active osteoblasts, endothelial cells, and bone mass was revealed, not with pore size [33]. Figures adapted from Mehdizadeh et al [29], Klenke et al [32], Kang et al [33]. 


\subsection{Pore structure}

3D pore structure including pore size, porosity, and interconnectivity greatly influence the vascularization of scaffolds [28,29]. Computer modelling studies have been used to gain information on the effects of pore variables in scaffolds on vascularization [29]. To predict the loss of mass in scaffolds, Mehdizadeh et al. [29] adapted a statistical-kinetic model developed by Metter et al. [30] (Fig. 3A). This model was extended to include bulk-degrading systems (polylactic acid (PLA)-b- Poly(ethylene glycol) (PEG)-b-PLA hydrogel network) which has hydrolyzed cross-link networks. In this model, the concentration of degradable PLA units within the undegraded hydrogel is given by: $[\mathrm{PLA}]=[\mathrm{PLA}]_{0} e^{-K^{\prime} t}$, where the initial and current concentration values of degradable PLA units are given by $[\mathrm{PLA}]$ and $[\mathrm{PLA}]_{0}$ with " $t$ " being the degradation time. From this, the probability of hydrolysis of any PLA unit (P) is found out and given by the formula: $P=1-[\mathrm{PLA}] /[\mathrm{PLA}]_{0}=1-e^{-K^{\prime} t}$. The fraction of polyacrylate chains $\left(\mathrm{F}_{\mathrm{PA}}\right)$ calculated by combinatorial statistics is given by $F_{\mathrm{PA}}=\left[1-(1-P)^{2}\right]^{N}$ followed by the calculation of the fraction of PLA-b-PEG-b-PLA segments released from the network: $F_{\mathrm{PEG}}=P^{2}+P(1-P) F_{\mathrm{PA}}$. From this, the model predicted the mass loss by the formula: $\%$ mass loss $=W_{\mathrm{PA}} F_{\mathrm{PA}}+W_{\mathrm{PEG}} F_{\mathrm{PEG}}$, where $W_{\mathrm{PA}}$ and $W_{\mathrm{PEG}}$ are the mass percentage of the hydrogel in the polyacrylate chains and cross-linked PLA-b-PEG-b-PLA segments. The critical time for reverse gelation can also be found using the equation: $t_{c}=\ln \left[(2 / N)^{1 / 2}\right] /-k$. Using these equations in the model, a 3D agent-based model was developed to simulate sprouting angiogenesis in porous symmetric scaffolds. Among others, large pore size, high porosity and large pore size distribution were found to be important to increase vascularization in porous scaffolds. In another modelling study, porosity was found to play a dominant role in affecting bone formation and angiogenesis across the pore size investigated (ranging from $180 \mu \mathrm{m}$ to $720 \mu \mathrm{m}$ ). The modelling study also predicted that the combined use of angiogenic factors in multi-layer scaffolds could promote angiogenesis and bone formation more readily than the single use of growth factors [31].

Pore size, among other properties of scaffolds, is the most widely studied parameter. Various compositions (bioceramics, polymers, composites) and different shapes (porous foam, fiber, hydrogel) have been used for the investigation of angiogenesis. For example, calcium phosphate (CaP), being a typical bone bioceramic, was prepared into particles with varying pore sizes (40-70, $70-140,140-210$, and $210-280 \mu \mathrm{m}$ ) and the ability to induce angiogenesis, vascularization and osseointegration was examined in critical-sized cranial defects in mice (Fig. 3B) [32]. The pore sizes over $140 \mu \mathrm{m}$ showed higher functional capillary density, suggesting the importance of pore size in vascularization and bone formation. CaP cylindrical blocks with pore sizes of 350-500 $\mu \mathrm{m}$ were also fabricated for the vascularization study (Fig. 3C) [33]. In this study, the microporous channel allowed blood flow in the graft even in
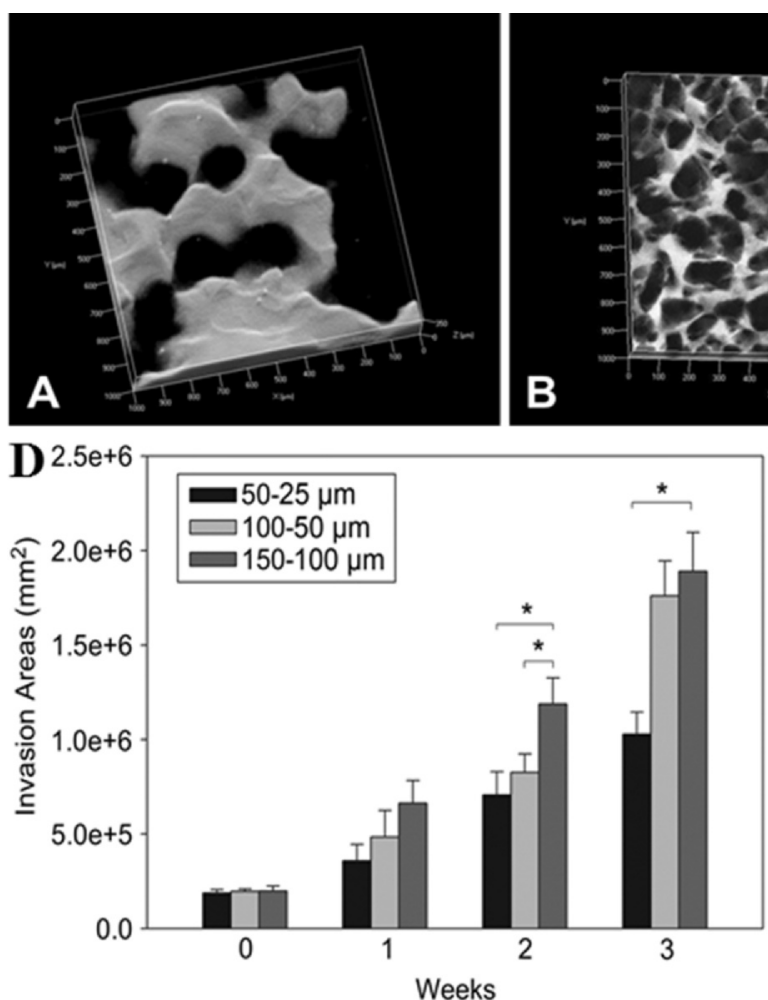
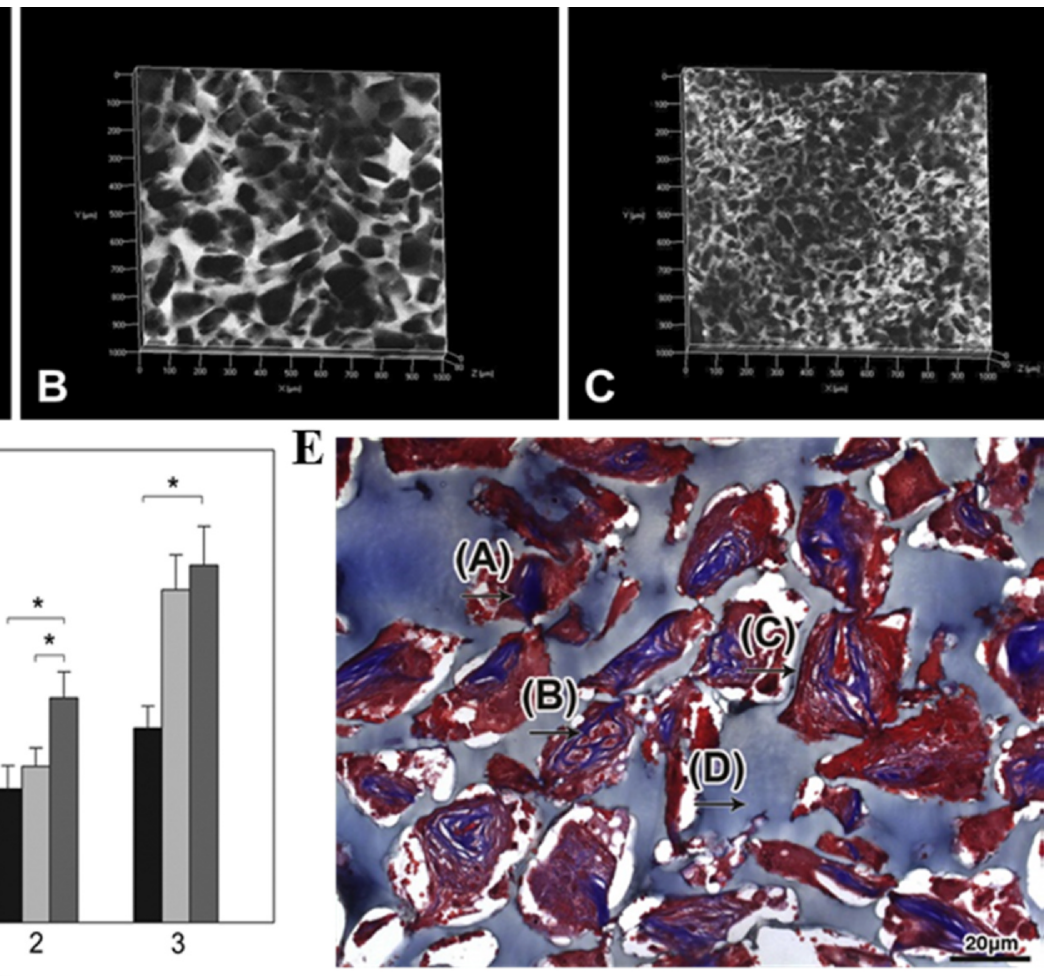

Fig. 4. Effect of pore size in porous hydrogels on new blood vessel formation. Porous PEG hydrogels, fabricated with different size of salt ranging from (A) 150-100, (B) 100-50, and (C) 50-25 $\mu \mathrm{m}$. The results from both (D) in vitro and (E) in vivo studies show that pore size mediates neovascularization. Hydrogels with pore sizes of 150-100 $\mu \mathrm{m}$ and 100-50 $\mu \mathrm{m}$ supported the invasion of endothelial cell/smooth muscle cell up to two or three weeks as well as the formation of collagen rich, vascularized tissue within the pores compared to 50-25 $\mu$ m pores. In (E), the mature collagen (blue, A) containing blood vessels (B), thin layer of inflammatory tissue (C), and hydrogel (D). Figures adapted from Chui et al., Biomaterials, 2011 [37]. 
the absence of endothelial cells. However, the presence of endothelial cells bolstered the blood flow through the channel. The interconnected macropores in the ceramic scaffold led to uniform deposition of collagen and easy penetration, and the endothelialized microchannel was further demonstrated to enhance the angiogenic sprouting and anastomosis.

Hydrogels are often used for the angiogenesis study because they can be developed to have tissue-level mechanical properties (low stiffness $0.1-10 \mathrm{kPa}$ ) and thus are considered to mimic the in vivo angiogenesis [34-35]. Porous hydrogels have the ability to induce angiogenesis from the surrounding environment [36]. PEG was used to test the role of pore size in vascular ingrowth (Fig. 4) [37]. For this, PEG gels with pore sizes of $25-50 \mu \mathrm{m}, 50-100 \mu \mathrm{m}$ and 100-150 $\mu \mathrm{m}$ were prepared and the tissue ingrowth was examined in 3D cell culture and rodent models. Results showed that vessel invasion was less in hydrogel with smaller pores (25-50 $\mu \mathrm{m})$ whereas the hydrogels with larger pores $(100-150 \mu \mathrm{m}$ and 50-100 $\mu \mathrm{m})$ supported deep vascular ingrowth with collagen rich tissue formation in vivo. Further, Tokatlian et al. [38] prepared porous hyaluronic acid with matrix metalloproteinase (HA-MMP) hydrogel with a pore size of either 100 or $60 \mu \mathrm{m}$. The effect of pores of hydrogel on angiogenesis was studied in a mouse wound closure model. The porous hydrogels loaded with pGFPluc reporter or pVEGF pro-angiogenic polyplex were implanted in a mouse wound healing model and the wound closure ability was examined. The macro-pores in hydrogels were shown to allow faster wound closure where $60 \mu \mathrm{m}$ hydrogel being superior to $100 \mu \mathrm{m}$ hydrogel. This study could establish the importance of pore existence and pore size in a soft hydrogel system.

Porosity is an important factor that determines the vascular ingrowth of scaffolding materials [39-41]. As the trabecular bone consists of a network of struts with interconnected porosities of 55-70\%, scaffolds are developed to have porosities in the range. The importance of pores in cell survival and angiogenesis has been well documented. In one study [42], large pores were prepared with
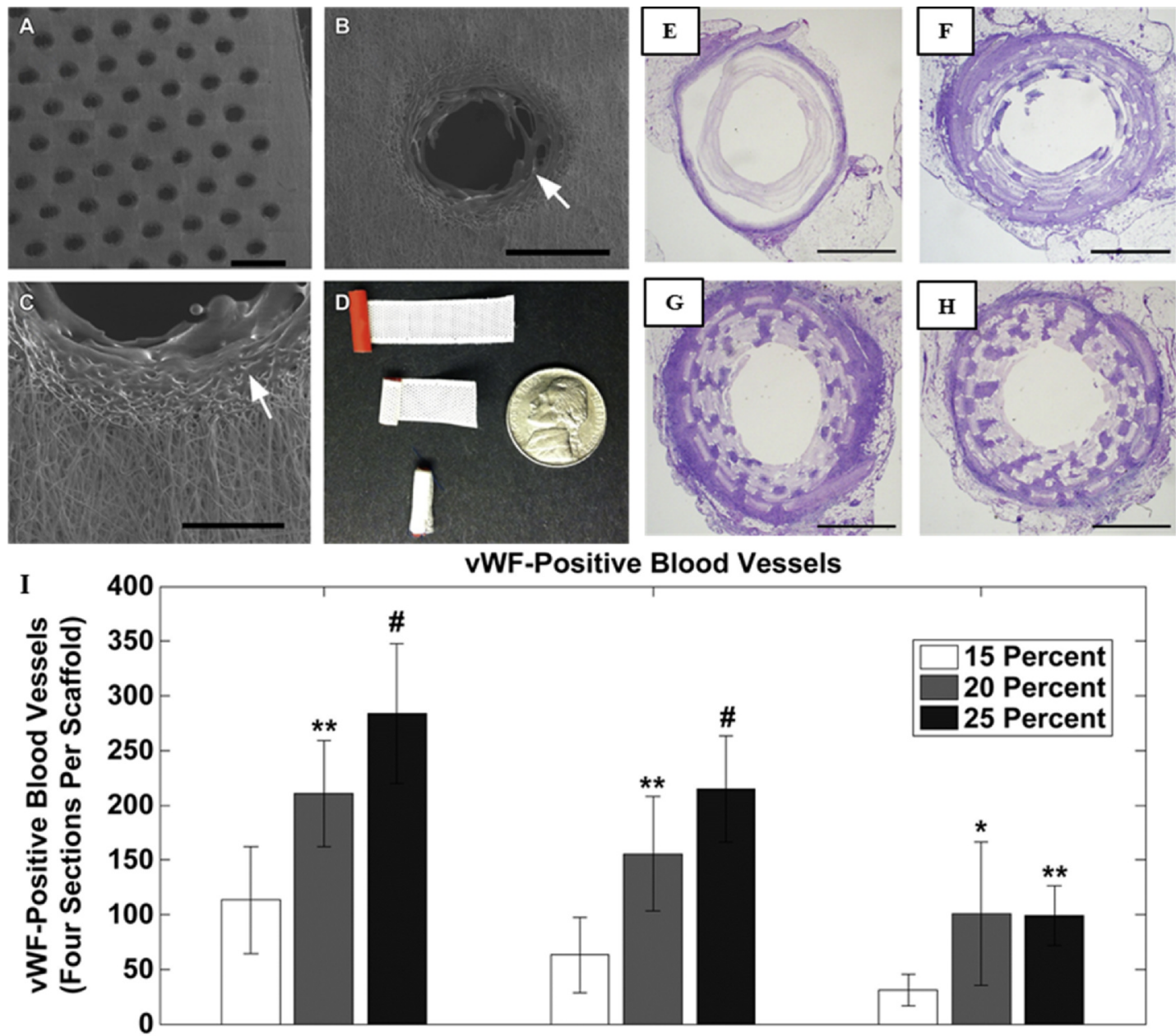

vWF-Positive Blood Vessels

Layers 5 and 6 (Outer) Layers 3 and 4 (Middle) Layers 1 and 2 (Luminal)

Fig. 5. Macroporosities in electrospun fibers effective for in vivo blood vessel infiltration. (A-C) Scanning electron microscope images of electrospun scaffolds with laser cut macropores (white arrow, $25 \%$ porosity, fiber diameters of about 3- $\mu \mathrm{m}$, and laser cut micropore diameters of $250 \mu \mathrm{m}$ ). (D) The wrapped scaffold was demonstrated for in vivo implantation. (E-H) Hematoxylin and eosin staining of implant sections after two weeks for 0\% porous (E), 15\% porous (F), 20\% porous (G), and 25\% porous (H). (I) Quantification of von-Willebrand Factor positive blood vessels. Statistical increases in blood vessel infiltration in electrospun scaffold existed in $20 \%$ and $25 \%$ macroporous scaffolds compared to $15 \%$ from outer, middle and luminal layers $(p<0.05, * ;<0.005$, **). Figures adapted from Walthers et al., Biomaterials, 2014 [44]. 
varying porosities (83.2\% and $86.5 \%)$ to mitigate the cell-mediated degradation of electrospun fibers. The cell migration was possible up to $4 \mathrm{~mm}$ under gravitational seeding, leading to a coverage of approximately $70 \%$ cross-sectional area. Another study attempted to investigate the relationship between fiber size and porosity [43]. When the fiber diameter increased, the pore size increased and at the same time the porosity also increased by $18 \%$. As a result, the cell infiltration and migration could be enhanced. In a similar study, Walthers et al. [44] prepared electrospun scaffolds with large pores (250 $\mu \mathrm{m}$ ) and macroporosities ranging from $15 \%$ to $25 \%$. They found that the blood vessel infiltration in the electrospun scaffolds were significantly higher in $20 \%$ and $25 \%$ macroporous scaffolds compared to $15 \%$ scaffolds based on the results on outer, middle and luminal layers (Fig. 5).

Pore interconnection influences angiogenesis. The interconnection size and degree (interconnectivity) determine the permeability of fluid and cell migration, and ultimately the area and number of blood vessels available inside the scaffold. The effects of pore interconnection on angiogenesis were examined by using CaP scaffolds which were developed to have various interconnection sizes $(100,120$ and $150 \mu \mathrm{m}$ ) with similar pore sizes (Fig. 6) [22]. An in vivo study in a rabbit femoral condyle showed that the $150 \mu \mathrm{m}$ interconnection size significantly improved the revascularization than the other sizes, highlighting the importance of pore interconnection size in vascularization. PEG hydrogels were also developed to have a controlled pore interconnectivity (Normal pore connectivity $=0.24$ (low interconnectivity) and 0.42 (high interconnectivity)) at a fixed range of pore size (130-150 $\mu \mathrm{m}$ ) [46]. Pores were generated using poly(methyl methacrylate) (PMMA) microspheres as a sacrificial template inside the PEG hydrogels. When implanted subcutaneously in rat, vascular tissue ingrowth occurred within the hydrogels. Of note, the ingrowth was found to vary with pore interconnectivity; hydrogels with higher interconnectivity $(0.42)$ showed complete vascularization within 3 weeks whereas those with lower interconnectivity $(0.24)$ had a very limited vascularization. This study established the importance of pore interconnectivity in influencing vascularization of hydrogel scaffolds.

\subsection{Physico-chemical properties}

The physico-chemical properties of biomaterials such as surface chemistry, topography, mechanical stiffness, and degradability have also been found to significantly influence the angiogenesis process.

\subsubsection{Surface chemistry}

Cells recognize the surrounding matrix through receptors (e.g., integrins and growth factor receptors). Thus, the surface of
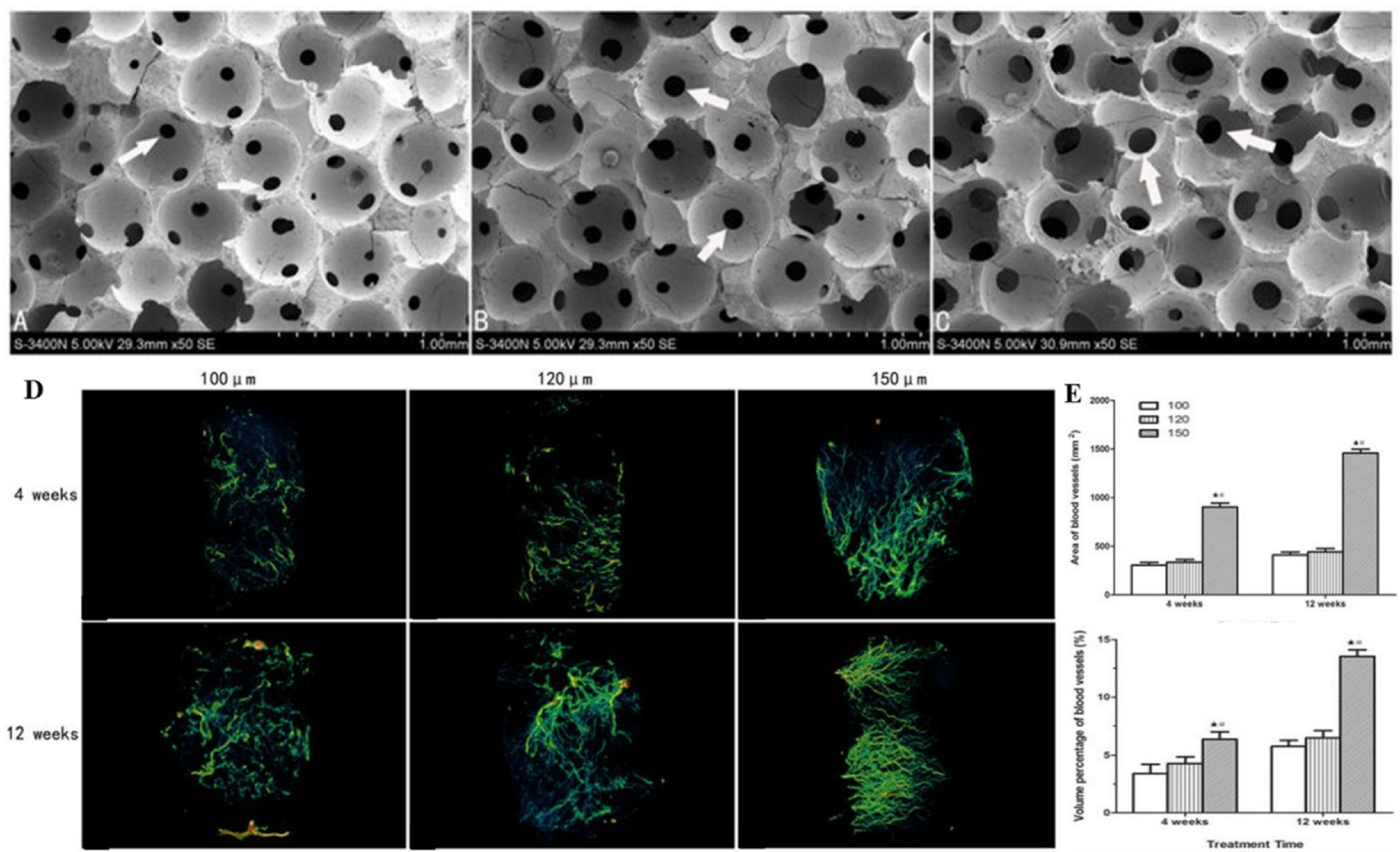

Fig. 6. Pore interconnection influences angiogenesis. The interconnection size and degree (interconnectivity) determine the area and number of blood vessels available inside the scaffold. (A-C) The scanning electron micrographs of porous $\beta$-TCP bioceramics with different interconnection sizes (white arrow) of (A) $100 \mu \mathrm{m}$, (B) $120 \mu \mathrm{m}$, (C) $150 \mu \mathrm{m}$ with same pore sizes $(300-400 \mu \mathrm{m}$ ). Scale bars: $1.00 \mathrm{~mm}$. (D) Typical $\mu$-CT 3D reconstruction images of new blood vessel ingrowth within scaffolds at 4 and 12 weeks after implantation using microfil contrast agent and (E) their quantitative analysis results. Statistical increase of blood vessel encapsulation existed in $150 \mu \mathrm{m}$ interconnection size. (\#, the difference attained a statistically significant increase compared to the $120 \mu \mathrm{m}$ group, $\mathrm{P}<0.05$; star, the difference attained a statistically significant increase compared to the $100 \mu \mathrm{m}$ group, $\mathrm{P}<0.05)$. Figures adapted from Xin et al., Sci Rep, 2015 [22]. 
biomaterials was widely functionalized with different chemistries and molecules to stimulate the receptor-mediated recognitions of the cells involved in angiogenic process (Fig. 7A) [47]. Early studies have modified the surface to conjugate growth factors more effectively. For example [48], poly-N-acetyl glucosamine nanofiber was employed to test EC motility which is important for angiogenesis and wound healing. They found that these fibers could induce integrin activation $\left(\alpha_{5} \beta_{1}\right.$ and $\left.\alpha_{v} \beta_{3}\right)$ leading to regulation of Ets1 transcription factor, and eventually wound healing ability. Metallic surface has also been functionalized with biomolecules to stimulate angiogenesis [49]. A covalent immobilization of RGD and YIGSR peptides on CoCr surface led to enhanced human umbilical vein endothelial cells (HUVECs) adhesion during coculture with smooth muscle cells, and further decreased thrombogenicity. Heparin is a well-known moiety to functionalize biomaterials surface with growth factors (e.g., VEGF) for angiogenesis [50]. Singh et al. [51] coated sub-micron thick layer of heparin onto polycaprolactone (PCL) scaffold that enabled VEGF loading. The scaffold loaded with VEGF was demonstrated to preserve sustained release of VEGF over 14 days and enhance angiogenesis in in vivo subcutaneous model in mice. Another study with tissue engineered vascular grafts (TEVGs) also functionalized heparin to immobilize VEGF or antibodies against CD34 by specific binding motifs in heparin interacting with (Fig. 7B) [52]. The modified TEVGs showed greater inner lumen diameter retention and endothelial nitric oxide synthase (eNOS) expression compared to unmodified TEVGs in inferior vena cava of mice. Heparin immobilization was also beneficial on collagen/hydroxyapatite (HA) nanocomposites in inducing osteogenesis and angiogenesis [53].

Not only the growth factor signaling, but the integrin-mediated stimulation of angiogenesis has also been documented. ECs recognize the underlying matrix through different sets of integrins which depend on the type of adhesive molecules. Fibronectin (FN) is well-known to influence the EC behavior through integrin signaling. In fact, one of the early studies linking biomaterials with FN and integrin was performed using self-assembled monolayer coatings with different functional groups $\left(\mathrm{OH}, \mathrm{COOH} \mathrm{NH}_{2}\right.$, and $\left.\mathrm{CH}_{3}\right)$ [54]. An erythroleukemia cell line expressing a single integrin $\left(\alpha_{5} \beta_{1}\right)$ was used to study the effect of surface properties on the cellular adhesion with adsorbed FN layers. The results showed a preferential affinity of FN to $\alpha_{5} \beta_{1}$ integrin, and a cell binding decrease on $\mathrm{NH}_{2}$ and $\mathrm{CH}_{3}$ owing to non-specificity. Also, the adsorbed $\mathrm{FN}$ density was changed depending on nano-scale roughness. This study established that the adhesion mechanism of $\alpha_{5} \beta_{1}$ integrin-expressing cells to adsorbed FN layer can be influenced by hydrophobicity, surface charge and roughness. Following this, an interesting study linking FN nanopatterns to integrin cell clustering was performed [55]. Using a nanosphere lithography (NSL)-based patterning technique, FN islands of large areas were created which were surrounded by passivated areas of PEG. The endothelial cell behavior on these nanopatterns were studied in terms of cell adhesion, spreading and proliferation. A time-dependent endothelial cell proliferation occurred with early proliferation independent of pattern size which was equivalent to that of high density nonpatterned surface. The late proliferation depended on average FN surface density, and pattern size below $\sim 0.05 \mu \mathrm{m}^{2}$. The FN nanoclustering induced less cell spreading initially than control, but the difference became less significant after $72 \mathrm{~h}$. These results highlight that the local FN density, adhesion size, and distance between adhesions are important in endothelial cell spreading. Of note, significantly increased cell spreading was found on nanoislands with sizes less than $0.1 \mu \mathrm{m}^{2}$, which implies the important dimension of adhesion sites encouraging further strategy to engineer biomaterials surface.

Recently, the integrin crosstalk with growth factors has been highlighted. An intriguing study by Moulisovã et al. [56] demonstrated the crosstalk of integrin and growth factor in ECs through FN-engineered surfaces. In fact, FN has specific sites for binding
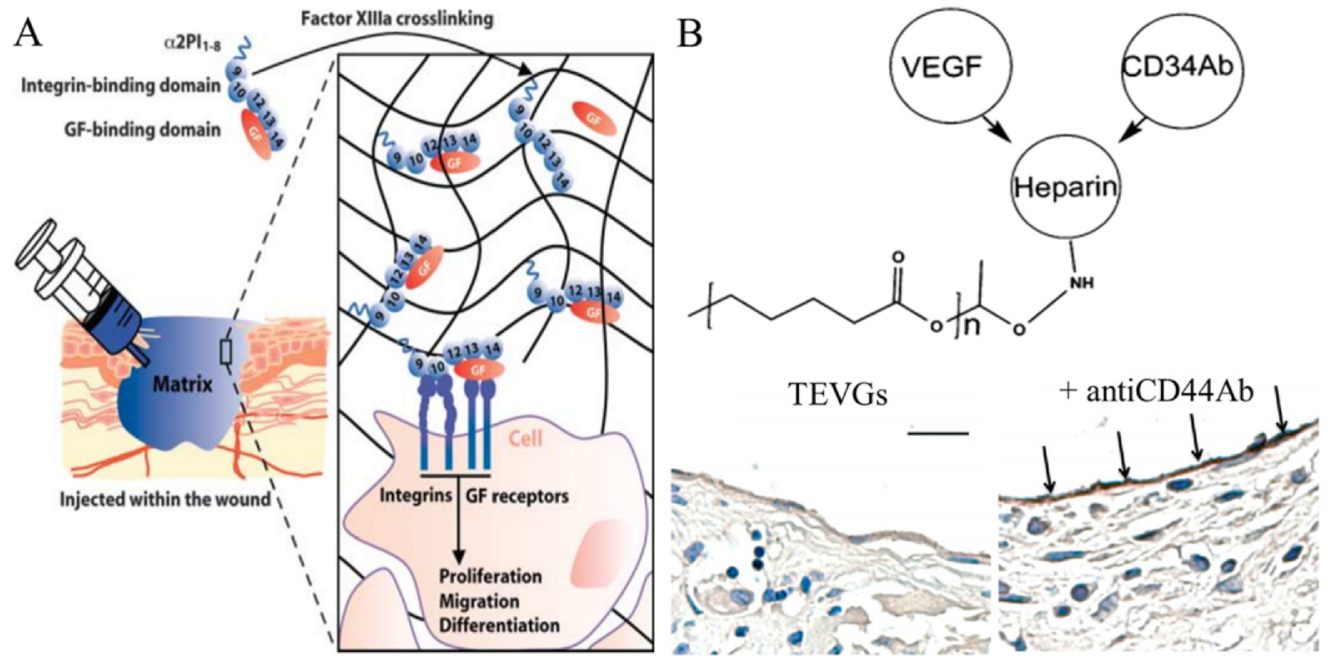

Fig. 7. Change in surface chemistry through molecular immobilization accelerates angiogenesis. (A) Recombinant fibronectin (FN) was engineered to comprise FN III12-14 (growth factors binding domain), factor XIIIa (fibrin matrix-binding sequence), and FN III9-10 (integrin-binding domain) and coated on fibrin matrix [47]. (B) Heparin was coated on biopolymer to immobilize VEGF or antibodies against CD34 (CD34; common marker of endothelial progenitor cells) for in situ endothelialization of endothelial and endothelial progenitor cells by specific binding motifs in heparin interacting with VEGF and anti-CD34Ab [52]. Endothelial cells (CD32 positive) were stained as brown color. Scale bar represents 20 $\mu$ m. Figures adapted from Martino et al., Science Translational Medicine, 2011 [47] and Melchiorri et al., Biomacromolecules, 2015 [52]. 
integrins (FN III9-10) and growth factors (FN III12-14) which are adjacent to encourage crosstalks between the two entities. They coated human plasma FN solution on spin-coated poly(methyl acrylate) (PMA) and poly(ethyl acrylate) (PEA) sheets, followed by incubation with VEGF (25 ng/ml). Of note, the FN organized on the PEA surface showed significantly enhanced VEGF signaling with integrin that on PMA surface. While both $\alpha_{5} \beta_{1}$ and $\alpha_{\mathrm{v}} \beta_{3}$ integrins interact with FNIII 10 region, this study showed $\alpha_{5} \beta_{1}$ integrin was more crucial for EC migration, proliferation and tubulogenesis (Fig. 8). This study highlights the importance of surface properties of biomaterials that can self-organize FN nanonetworks properly to present integrin and growth factor binding regions which ultimately promote angiogenesis.

\subsubsection{Surface topography}

Surface topography has been shown to impact different cell behaviors, such as stem cell specification, myoblast migration, osteoblast maturation, and even angiogenesis. The surface topographical properties engineered in biomaterials generally scale from tens of micrometers down to tens of nanometers [57]. In fact, various topographies (pillars, pits, grooves/gratings, tubes, fibers, wires, roughness, and islands) have been introduced for specific purposes [58]. While the micrometer-scale influences the gross motility of cells, the nanometer-scale determines the sensing of receptor molecules (e.g., integrins). Among other cells, EPCs responded to ridge-groove grating of $600 \mathrm{~nm}$ in depth and $1200 \mathrm{~nm}$ in period through alignment, elongation, reduced proliferation, and enhanced migration (Fig. 9) [59]. EPCs cultured on nanogroove substrate induced supercellular band structures after $6 \mathrm{~d}$ and enhanced tubular formation and organization on matrigel coated nanogroove. Similarly, osteoblasts cultured on differently engineered surface topologies were shown to imply the altered angiogenesis through secretion of different angiogenic molecules [60]. The osteoblasts cultured on Ti discs with various roughness showed stimulated $\alpha_{2} \beta_{1}$ integrin [61] where integrin $\beta_{1}$ was found to be the prime molecule in recognizing Ti surface roughness [62]. It is worth noting that $\alpha_{2} \beta_{1}$ signaling also regulates VEGF-A and fibroblast growth factor (FGF)-2 from osteoblasts [63-64], implying the topography-associated stimulation of integrin $\beta_{1}$ in osteoblasts is linked with angiogenesis. Furthermore, integrin $\alpha_{2}$ signaling was also found to be responsible for the increased production of angiogenic factors. Titanium surfaces with different microtopographies were shown to alter the response of osteoblasts in secreting VEGF-A, FGF2 , epidermal growth factor (EGF) and Ang-1 through integrin $\alpha_{2}$. Collectively, the integrin $\alpha_{2} \beta_{1}$ signaling may play an essential role in the production of angiogenic factors in osteoblasts which is triggered by surface topography.

A
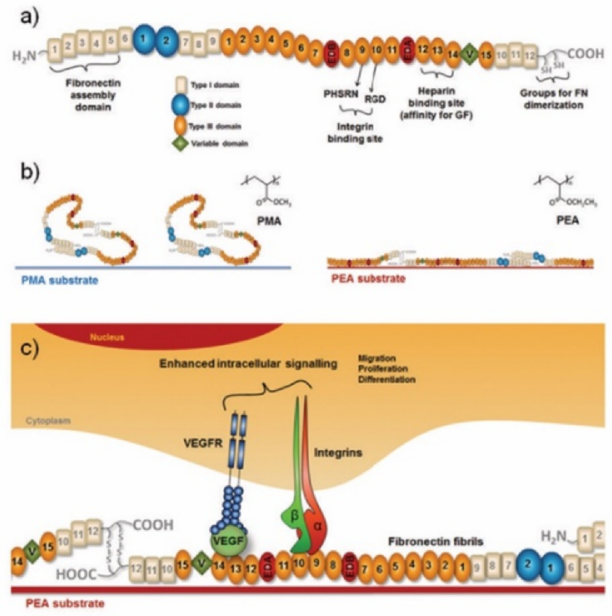

C

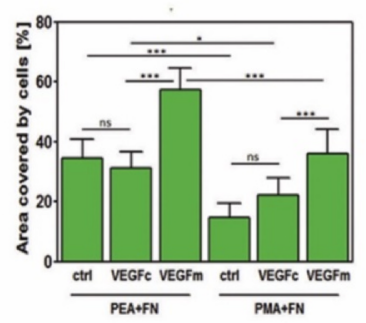

B
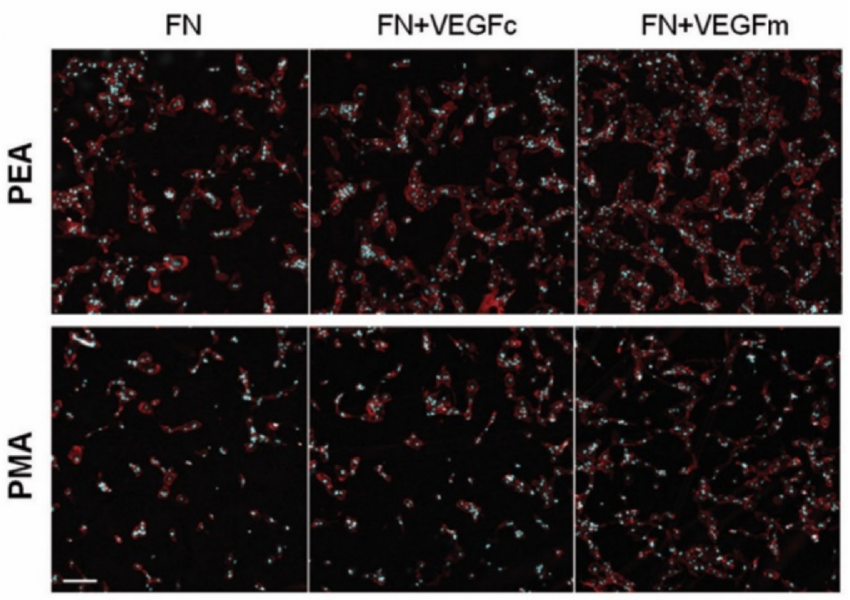
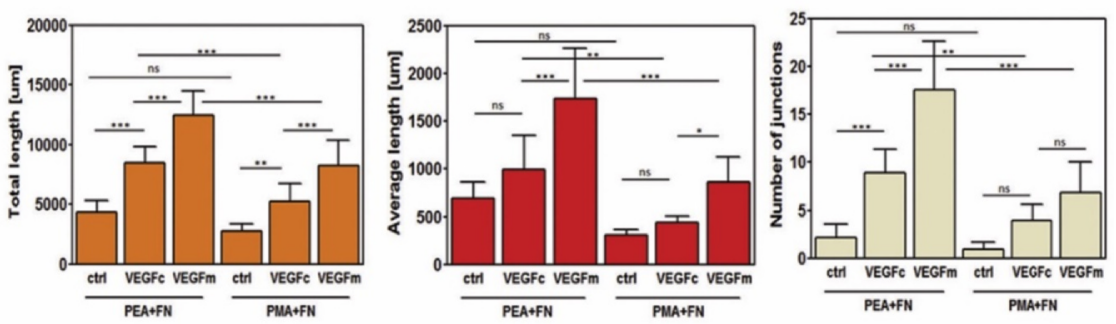

Fig. 8. Surface chemistry-modulated angiogenesis by integrin-growth factors receptor crosstalk. Different matrices alter the fibronectin presentation and its co-signaling with growth factor receptor VEGFR in angiogenesis. (A) Schematic showing the synergistic signaling of VEGF and integrin with fibronectin which leads to vascularization of HUVEC on poly(ethyl acrylate) (PEA) substrate (vs. poly(methyl acrylate) (PMA)); (B) FN + VEGFc coated PEA shows higher degree of aligned HUVEC structures in comparison to other samples; and (C) total cell area coverage, length, average length, junction of HUVEC networks are higher in PEA networks when compared with PMA substrates. Adapted from Moulisová et al. Biomaterials, 2017 [56]. 

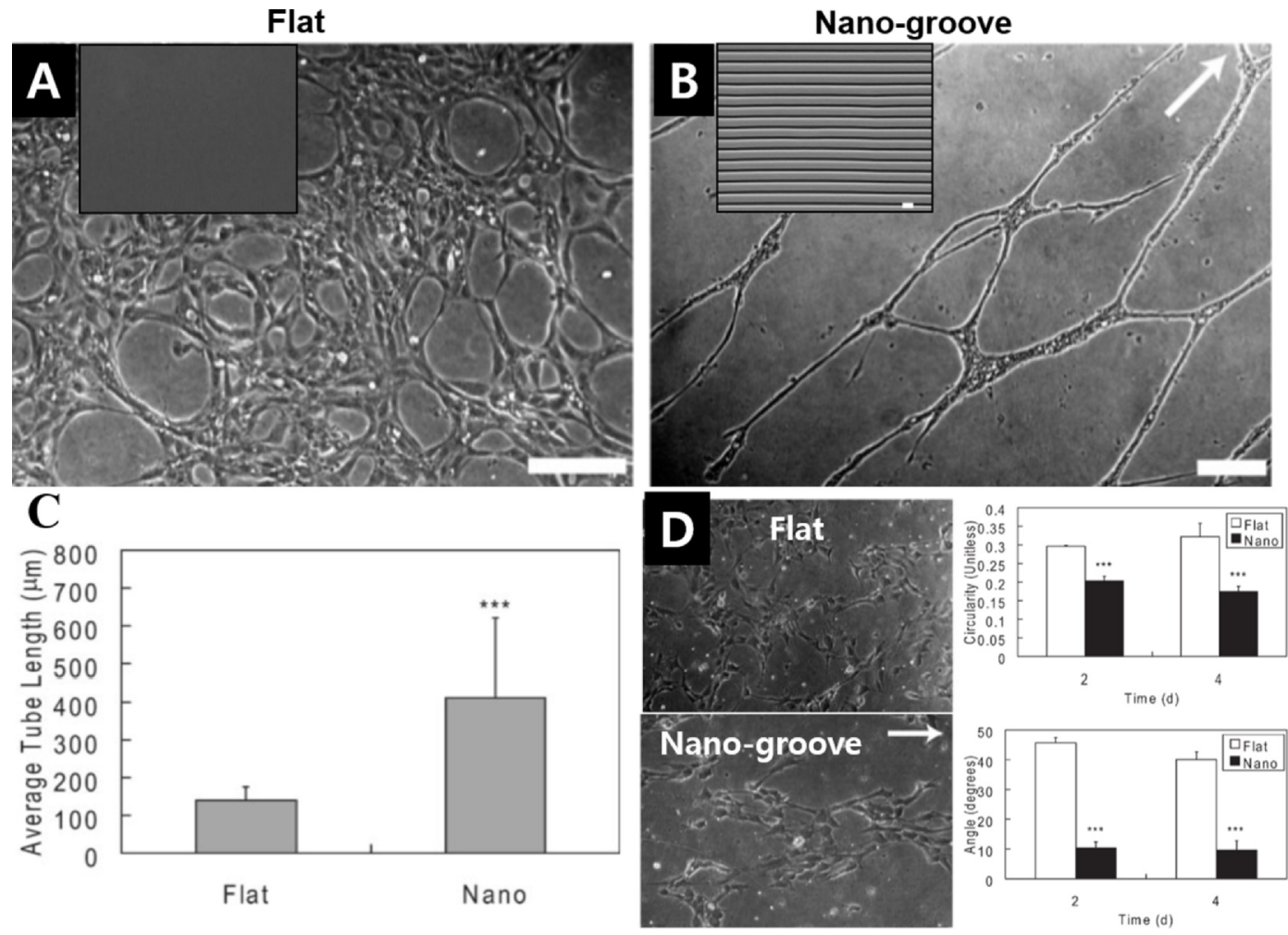

Fig. 9. Surface groove topography governs endothelial progenitor cell (EPC) organization. Nano topographical features on substrates could promote the EPC tubular formation (A-C, insert revealing image of substrate) and organizations (D; circularity and direction) into well-defined vascular structures in vitro. Scale bars in A\&B are $200 \mu \mathrm{m}$. Adapted from Christopher et al., Advanced Materials, 2008 [59].

Not only the angiogenic stimulating roles played through osteoblasts, but also the direct effects of surface topography on ECs can also be reasoned because the nanoscale-sensing though integrins as well as the cytoskeletal rearrangement and migration along the micro-scaled surface are both important for angiogenic events of ECs [65]. For example, the sensitivity of different cells (ECs, SMCs smooth muscle cells, and fibroblasts) to surface topography in the micro and nano range was studied [66]. Grooved
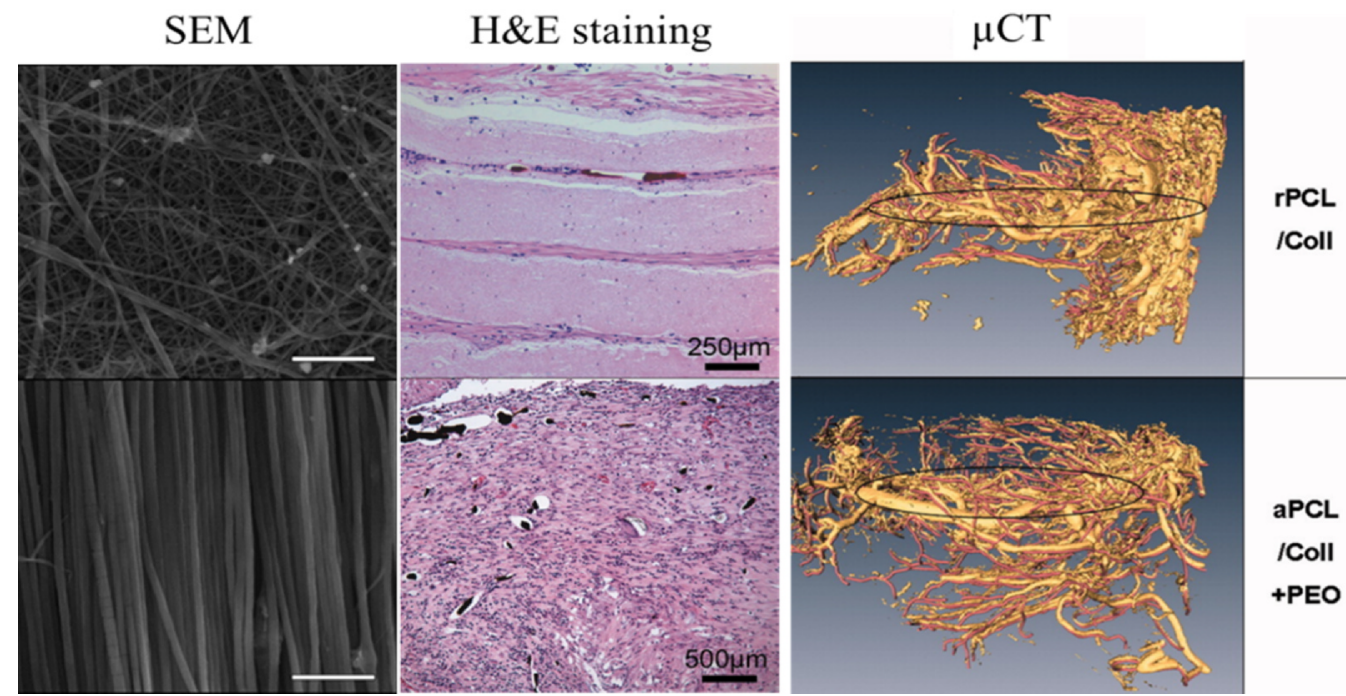

Fig. 10. Alignment of electrospun 3D fibers accelerates in vivo vascularization. Randomly spun PCL/collagen blend (rPCL/Col1) and parallel aligned PCL/collagen blend/PEO scaffolds (aPCL/Col1 + PEO) were fabricated and implanted in vivo (AV-loop model) for investigating numbers and patterns of sprouting vessels inside the constructs using histology and microCT analysis. Aligned electrospun 3D fibers accelerated numbers and patterns of sprouting vessels compared to randomly spun counterpart. Adapted from Klumpp et al., J Biomed Mater Res A, 2012 [68]. 
polydimethylsiloxane (PDMS) substrates with lateral dimension of 2-10 $\mu \mathrm{m}$ and depth of 50-200 nm were prepared and the contact guidance alignment and directed migration of cells were examined. The contact guidance of cells significantly increased on substrates with smaller lateral dimensions or deeper grooves. Also, a minimum groove depth of $50 \mathrm{~nm}$ was necessary for fibroblasts to elicit an orientation response and a change in cell shape while ECs and SMCs required much deeper grooves (twice) for the same response. Fibroblasts were strongly aligned and had a faster directed migration compared to ECs and SMCs which is beneficial during wound healing. In another work [67], endothelial cells were seeded onto micro- to nano-grooved PDMS substrates (200 nm, $500 \mathrm{~nm}, 1 \mu \mathrm{m}$, and $5 \mu \mathrm{m}$ depth) which were precoated with $\mathrm{FN}$, and the endothelial behaviors were investigated. The cell alignment increased with channel depth by factors of 1.7-2.0 during $48 \mathrm{~h}$. Even with those studies, the effects of surface topography on endothelial cells have mostly been examined in 2D conditions, thus limiting the interpretation of phenomena in 3D environments. One intriguing study was carried out to examine the effects of pore surface pattern in 3D. Aligned and random electrospun fibers made of PCL/collagen were prepared and the vessel formation in vivo was monitored (Fig. 10) [68]. Results showed that significantly smaller number of sprouting vessels in general but earlier vascularization were noticed in the aligned fiber with respect to the random fiber, and the study established the role of pore surface alignment (pattern) in determining the 3D formation of vessel trees.

\subsubsection{Stiffness}

Stiffness is an important biophysical cue that determines cellular fate including those related with angiogenic events [69-71]. Cells constantly sense the stiffness of their underlying substrate by forming focal adhesion complexes and transferring actin-myosin contractile force, and then feedback through various intracellular signaling that have implications in developmental biology, cellular differentiation, tissue regeneration, and pathological disease [72]. In fact, the importance of stiffness in dictating cellular behaviors has been well documented with different types of cells including those with multipotent (mesenchymal stem cells (MSCs), neural stem cells) or pluripotent capacity (embryonic stem cells), or those in pathological conditions (cancer cells) [73-75].

As to the ECs, some of the recent works have reported significant roles of the matrix stiffness in vascular networks. ECs cultured on type-I collagen gels formed networks on both soft and stiff gels, however, the EC networks on the stiff gels had more pronounced actin stress fibers [76], leading to much stiffer cellular characteristic on stiff substrate than on soft substrate (Fig. 11A-F). Stevenson et al.
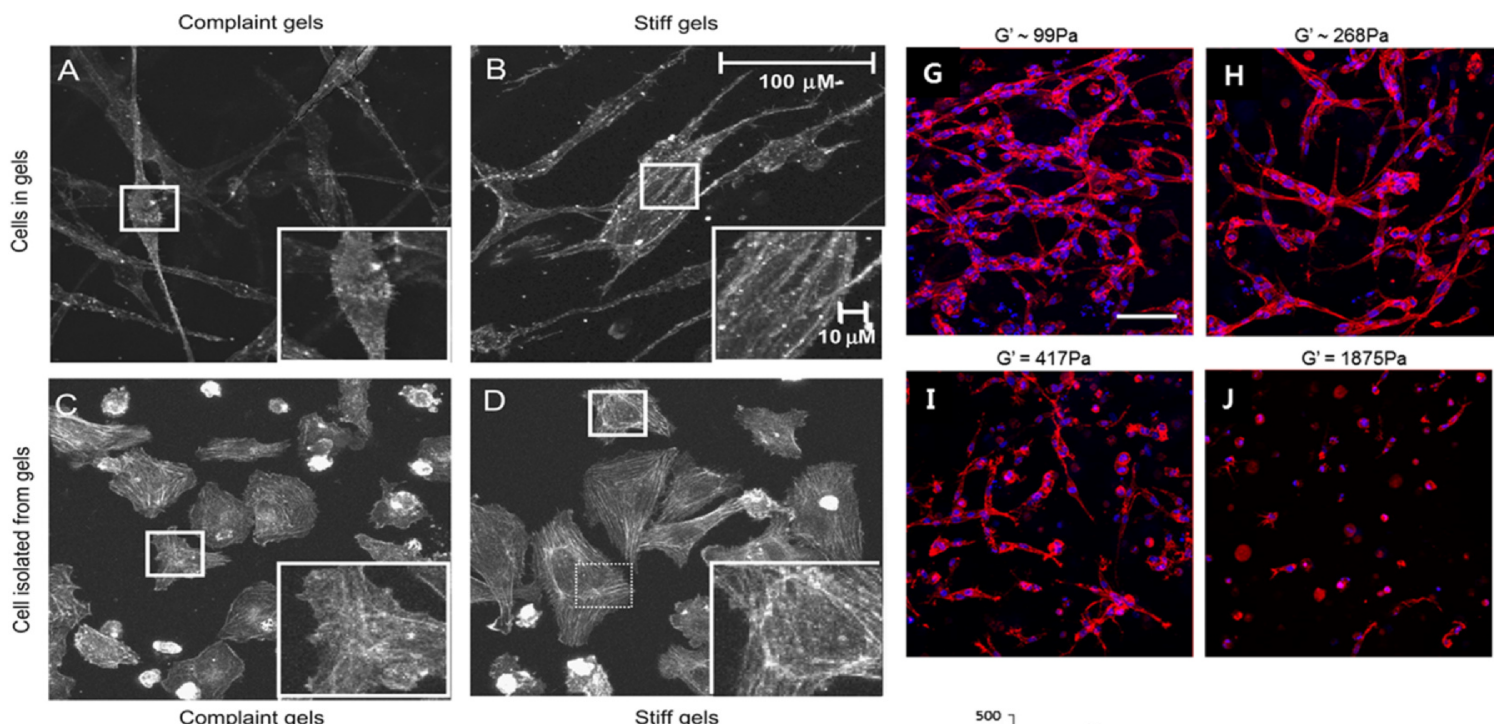

$\mathrm{E}$

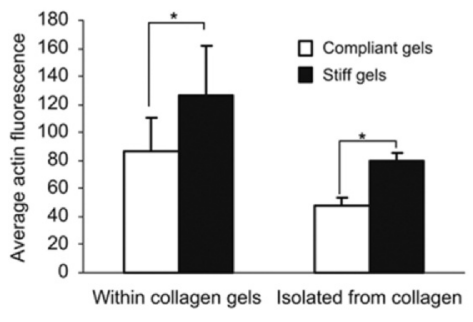

$\mathrm{F}$

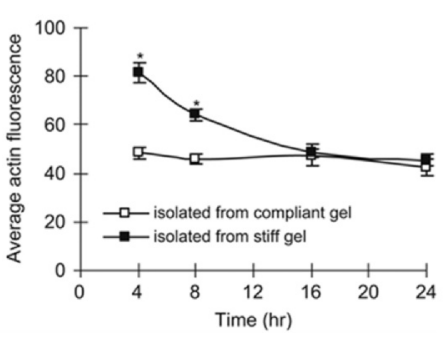

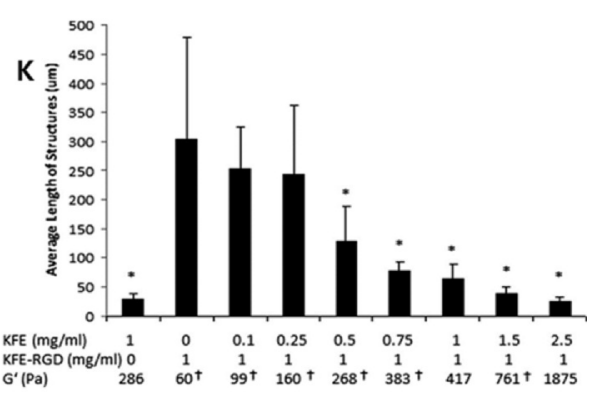

Fig. 11. Stiffness of biomaterials influences endothelial cell behaviors. (A-F) Average actin fluorescence of endothelial cells is greater in stiff gels (polyacrylamide gels; $9000 \mathrm{~Pa}$ ) when compared to compliant gels (1700 Pa). Adapted from Byfield et al., J Biomechanics, 2009 [76]. (G-K) Endothelial cells spread less on self-assembling peptide (KFE) matrix as matrix stiffness is increased. The removal of RGD in matrix nullified the effects of stiffness, supporting the adhesion-dependent process is the key for cell-dependent sensing of the underlying matrix stiffness. Scale bar is $100 \mu \mathrm{m}$. Adapted from Stevenson et al., Acta Biomater, 2013 [77] 


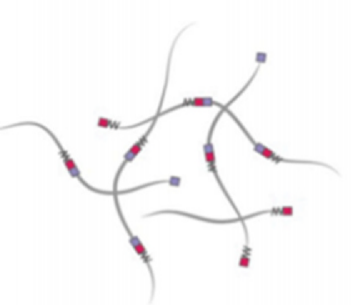

MMP-sensitive TG-PEG

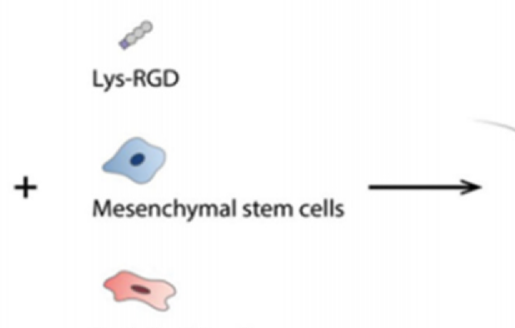

Endothelial cells

\section{F-actin}
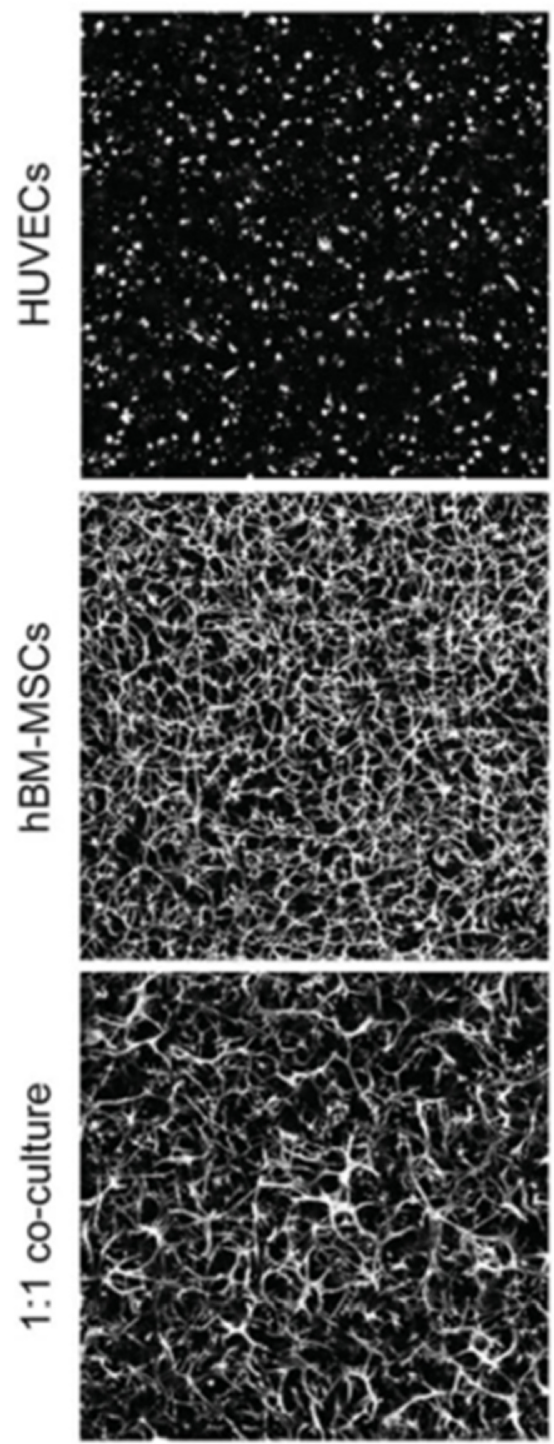

CD31
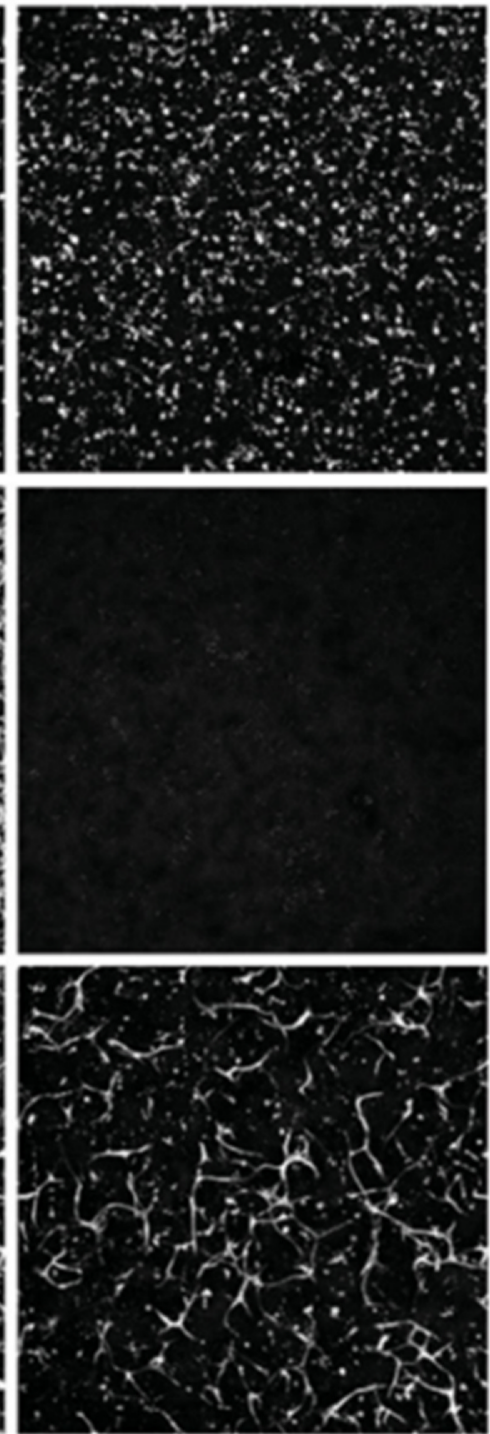

F-actin CD31
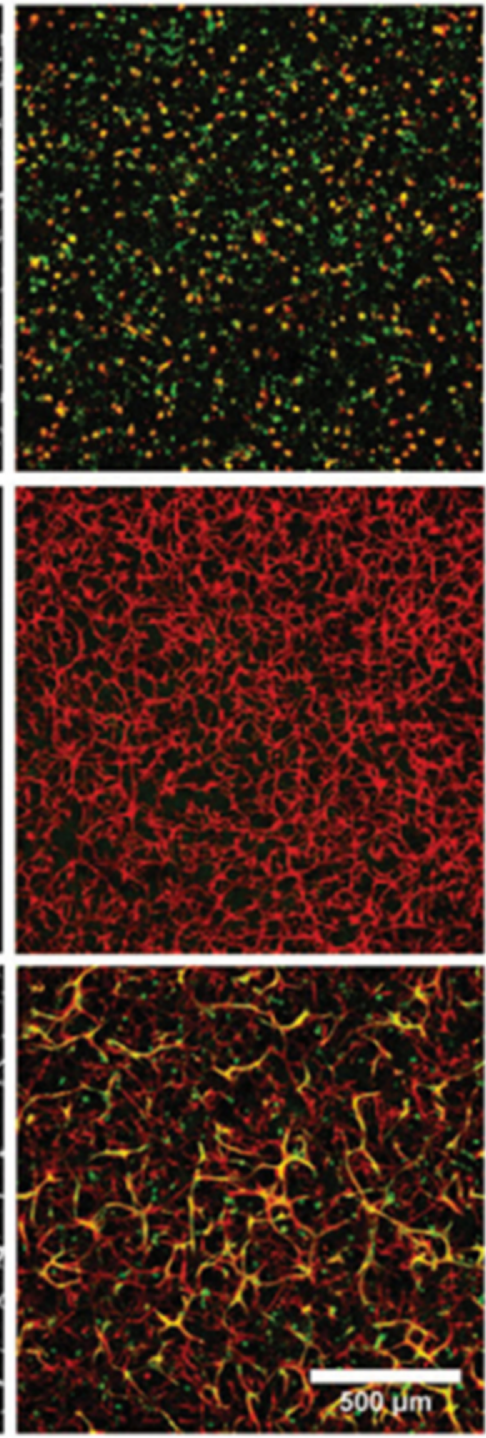

Fig. 12. Tailoring cell-mediated degradation to control angiogenic events in hydrogel. Designing bio- and cell-degradable biomaterials to promote

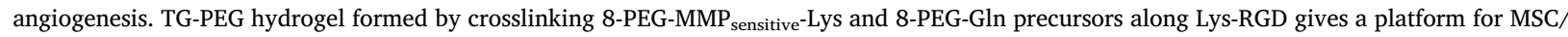
HUVEC to attach and proteolyze the matrix during microvascularization. MSCs enable HUVECs in self-organized cocultures to build lumenized microvascular structures in synthetic PEG hydrogels. Adapted from Blache et al., Adv Health Mater, 2015 [86]. 
[77] introduced a density of integrin bindings along with stiffness to study vascular network formation in a self-assembled peptide hydrogel (Fig. 11G-K). Two peptide sequences with one peptide incorporated with RGD formed the hydrogel with encapsulated HUVECs and hMSCs. When the stiffness of the hydrogel increased, the HUVECs failed to form microvascular networks while the hMSCs spreading increased. Although stiffness showed cell-dependent phenomena in the hydrogels, the removal of RGD nullified the effects, supporting again the adhesion-dependent process is the key for cell-dependent sensing of the underlying matrix stiffness.

The effects of matrix stiffness on early bone healing, where ECs interplay with osteoblasts and angiogenesis is important to relay osteogenesis, were further investigated. For this, HUVECs were cultured on collagen-coated polyacrylamide (PAAm) hydrogels with different stiffness levels ( 3 or $30 \mathrm{kPa}$ ) [78], and then genes and proteins involved in angiogenesis and osteogenesis were analyzed at different time frames (up to 7 days). The ECs cultured on a stiffer matrix exhibited down-regulation of the VEGF receptor-2 protein with concurrent over-expression of caveolin-1, WNT2, bone morphogenetic protein 2 (BMP2), and bFGF, which suggests that matrix stiffness influences ECs to express pro-angiogenic or osteogenic factors. Thus, the stiffness needs to be carefully designed to provide matrix conditions favorable for angiogenesis and bone healing at specific time points.

\subsubsection{Degradability}

Degradability of biomaterials is important as it determines both the biochemical and mechanical properties over time which ultimately influences the angiogenic process [79]. For successful bone growth, the dissolution rate of the implanted biomaterials must match the new bone growth rate of the host tissue. This research area has been comprehensively studied for various biomaterials such as bioceramics, composites, and hydrogels [80-83]. Here, we focus on cell-mediated degradation to control angiogenic events. The ECM density regulates how cells move inside the hydrogel. When the density is high, the cells produce enzymes to degrade the matrix which makes it easy for cell migration. On the contrary, if the ECM density is low, the cells secrete the matrix that helps them to get a firm grip for migration [84]. This phenomenon of the cells to sense the surrounding and secrete matrix dissolving enzymes has been targeted to engineer tailored degradable hydrogels for vascularization. One such effort was the cell-demanded liberation of VEGF-121 from fibrin gel matrices to control vascularization [85]. Here, a VEGF variant, $\alpha_{2} \mathrm{PI}_{1-8}-\mathrm{VEGF}_{121}$ was engineered in a fibrin gel matrix, and the angiogenesis was examined in chicken chorioallantoic membrane (CAM) assay and adult mice. The covalently bound $\alpha_{2} \mathrm{PI}_{1-8^{-}}$ $\mathrm{VEGF}_{121}$ was released from the fibrin matrix only when cells released MMPs or plasmin due to a remodeling activity. Results showed superior neovessel formation without leakage whereas the wild-type VEGF $_{121}$ produced chaotic changes in the capillary plexus. In a similar approach, vascularized bone construct was prepared by coculturing MSCs with HUVECs in a proteolytically cleavable PEG hydrogel [86], where the PEG was conjugated with TG-MMP-Lys peptide through a Michael-type addition reaction to impart degradable properties with tailed mechanical properties and cellular signaling (Fig. 12). The degradation kinetics of a 3D matrix should be carefully designed as the angiogenesis event involving EC migration and vasculature formation is a dynamic process where cells degrade the surrounding networks while depositing new proteins. In this sense, the stress relaxation, a representative time-dependent property of viscoelastic matrices, has recently been considered as the key regulator of cell fate, including MSC specification and chondrocyte function $[87,88]$. Therefore, control of the viscoelastic behaviors of biomaterials, particularly hydrogels, may be a future
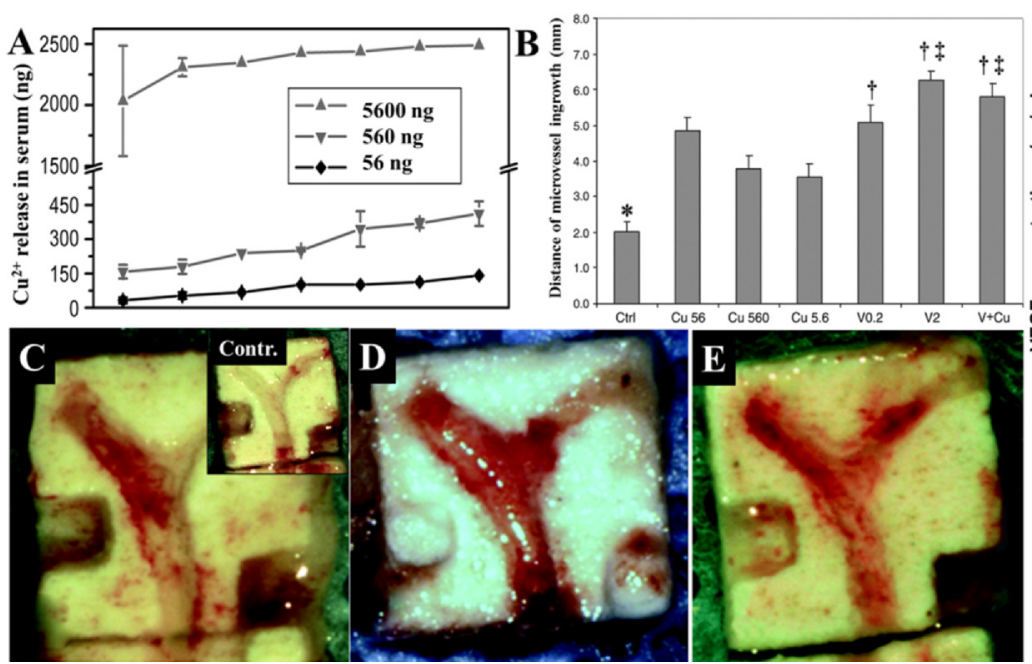
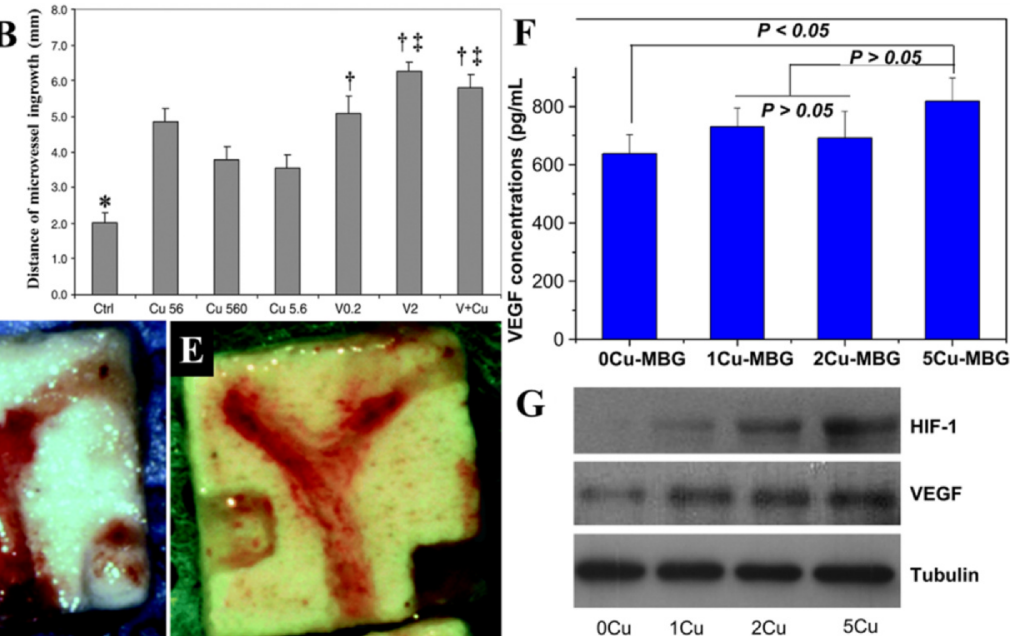

Fig. 13. Copper-doped scaffolds for vascularization via the delivery of ions. (A-E) Copper-doped calcium phosphate bioceramic scaffold with carved Y-shape, capable of releasing copper ions (A), shows excellent microvessel ingrowth. Representative macroscopic views of one half of bioceramic implant at retrieval. The conditions are control implant (insert, C); implants loaded with 56 ng copper (C); loaded with $0.2 \mu g$ VEGF (D); loaded with a combination of $0.2 \mu \mathrm{g}$ VEGF and $56 \mathrm{ng}$ copper (E). From these views, migration of the vascularized tissue was quantified (B). *Control condition significantly different ( $p \leq 0.05$ ) compared with the other conditions; ${ }^{\dagger}$ compared with Cu 5.6; and compared with Cu 560. Adapted from Barralet et al., Tissue Eng A, 2009 [106]. (F \& G) Copper containing mesoporous bioglass (MBG) scaffolds with large pores (300-500 $\mu$ ) developed for multifunctions in bone repair, including angiogenesis. (F) The secretion of VEGF was markedly elevated when hBMSCs were cultured in 5Cu-MBG extracts compared to those in Cu free -MBG extracts. (G) Western blotting analysis confirmed the increasing trend of VEGF and HIF-1 $\alpha$ expression in hBMSCs cultured in 5Cu-MBG extracts. Adapted from Wu et al., Biomaterials, 2013 [104]. 
exciting approach to control angiogenesis.

\section{Controlled delivery and presentation of angiogenic molecules}

Along with the tailoring of intrinsic properties of biomaterials, such as pore architecture, surface chemistry, topography, and bulk stiffness, the controlled delivery of angiogenic molecules through biomaterials is a key strategy to stimulate angiogenesis. Representative angiogenic molecules introduced to the scaffolds are metallic ions and growth factors.

\subsection{Release of angiogenic ions}

Angiogenesis and bone formation are significantly influenced by the metallic ions, such as boron (B), magnesium (Mg), strontium (Sr), copper (Cu), cobalt (Co), titanium (Ti), zinc ( $\mathrm{Zn}$ ), and silicate [89]. Therefore, various bone regenerative scaffolds have been developed to incorporate such ions within their structure [90]. Apart from being pro-angiogenic, some of the ions (e.g. $\mathrm{Cu}, \mathrm{Zn}, \mathrm{Ag}$ ) provide additional therapeutic effects such as anti-bacterial activity and anti-inflammation. The introduction and delivery of ions through biomaterials is thus considered as a growth factor-free approach to stimulate angiogenesis in bone repair. We discuss some biomaterials developed to deliver the ions and their angiogenic effect and the resultant tissue healing capacity observed in vitro and in vivo with some exemplar cases.

\subsubsection{Copper}

Copper ion has the ability to upregulate VEGF expression and promote neovascularization leading to faster wound healing in rats [91,92], and proliferation of ECs in in vitro culture [93]. Copper ion mimics hypoxia by stabilizing expression of hypoxic inducing factor (HIF-1 $\alpha$ ), thereby stimulating VEGF genes and others [93,94]. Hypoxia is an important factor that induces ECs toward the formation of functional vasculature supporting blood supply $[95,96]$. The in vitro and in vivo effects of HIF-1 $\alpha$ on osteogenesis and angiogenesis have been in-depth studied [97-103]. Wu et al. [104] incorporated Cu within mesoporous bioactive glass (MBG) scaffolds with large pores $(300-500 \mu \mathrm{m})$ and well-ordered mesopores $(5 \mathrm{~nm})$, and investigated the effects of Cu-induced hypoxia on hMSCs. The Cu-MBG scaffolds showed improved angiogenesis and osteogenesis with respect to Cu-free MBG (Fig. 13F\&G). Apart from stimulating osteogenesis, the antibacterial property of Cu-MBG scaffolds was firmly established against various bacterial strains ( $E$. coli, S. aureus and S. epidermis) [105]. Having confirmed the angiogenic and antibacterial properties, scaffolds containing copper ion were proposed to be useful in wound healing applications. For this, Barralet et al. [106] used copper ion in brushite bioceramics and investigated the effects of copper sulfate in comparison with the VEGF and copper ion/VEGF groups (Fig. 13A-E). Among the different doses of copper, they found that $56 \mathrm{ng}$ of copper per scaffold provided an optimal angiogenesis during tissue ingrowth in vitro and again that 10 -fold increase $(560 \mathrm{ng})$ in the copper dose for the in vivo ingrowth of wound tissue. Thus, the copper-loaded brushite scaffolds were demonstrated to have enhanced angiogenesis and wound healing.
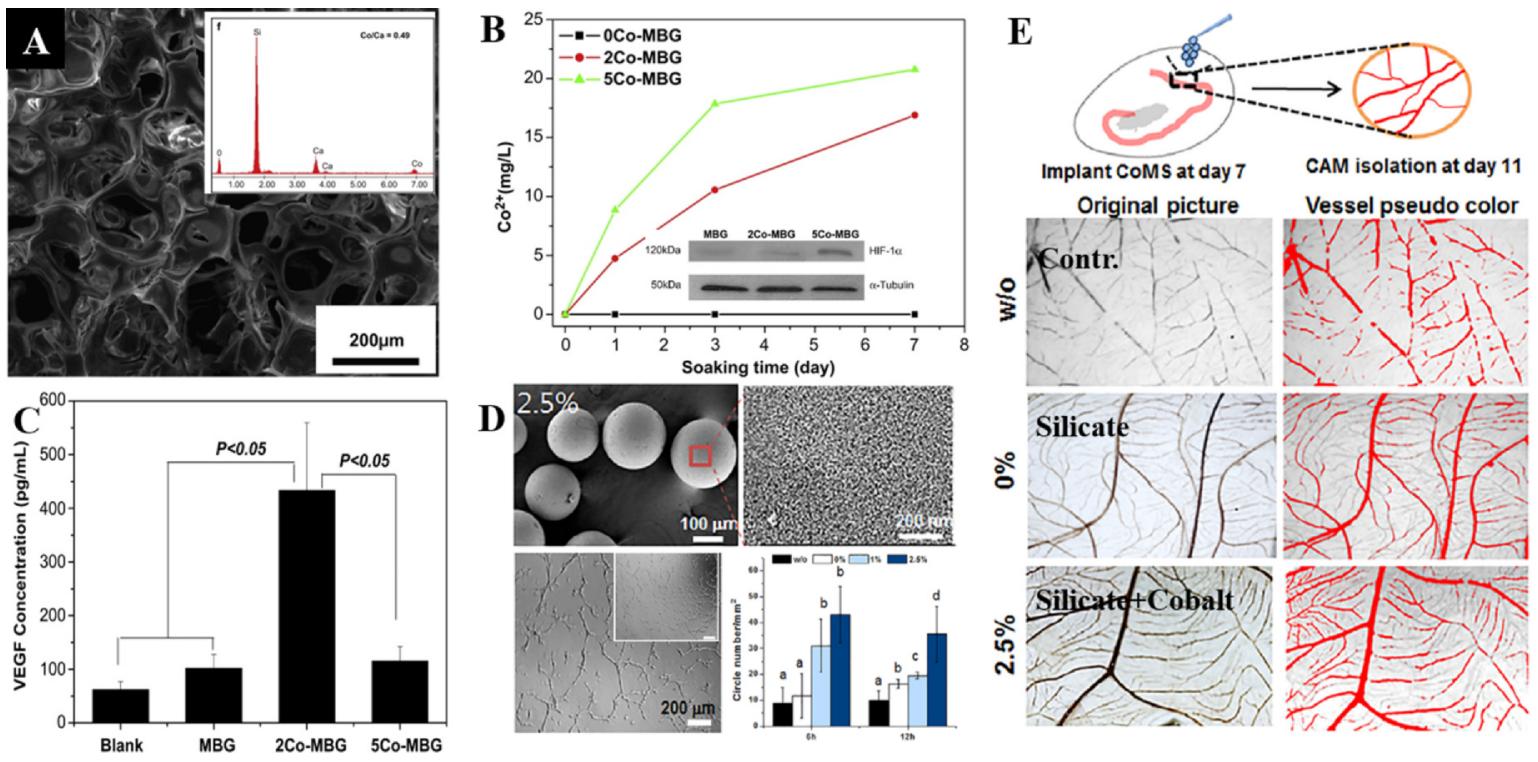

Fig. 14. Cobalt-ion induced vascularization through biomaterials. (A) Cobalt-doped (2-5\%) mesoporous bioglass scaffold, (B) capable of releasing cobalt ions in culture media, for bone angiogenesis was revealed in hMSCs by (B, insert) HIF-1 $\alpha$ protein level and (C)VEGF secretion. Adapted from Wu et al., Biomaterials, 2012 [108]. (D; upper) Dual-ion (cobalt and silicate) delivery cobalt-doped-silicate-microspheres ( $\sim 300 \mu \mathrm{m})$ with mesoporous surface was fabricated, which synergistically upregulated (D; bottom) HUVECs' in vitro tubular formation and (E) in vivo neo-vascular formation using a chicken chorioallantoic membrane model. Adapted from Khaliun et al., Acta Biomater, 2019 [112]. 


\subsubsection{Cobalt}

Cobalt is another ion that has the ability to induce HIF-1 $\alpha$, and thereby aids in vascularized bone formation in vivo [107]. Cobalt ion, when introduced into MBG, is potent to initiate vascularized ingrowth during bone formation. In one study, MBG sczffolds incorporated with $<5 \%$ Co significantly induced hypoxia, thereby enhancing VEGF protein secretion and HIF-1 $\alpha$ expression along with increased expression of osteogenic genes (Fig. 14A-C) [104,108]. The incorporation of cobalt to melt bioactive glasses also enhanced the bone forming ability in vitro. Hoppe et al. [109] fabricated Co ion releasing 1393 bioactive glasses and performed in vitro studies with simulated body fluid. They found that the Co-containing bioactive glass surface was rapidly ( $<7$ days) covered with an apatite-like layer and the Co ions could be released sustainably to therapeutically relevant levels. Cobalt-doped bioactive glass, when combined with organic materials such as collagen, was further used to enhance bone regeneration. Quinlan et al. [110] developed collagen/glycosaminoglycan composite scaffolds with impregnated cobalt-doped bioactive glass. The Co-doped bioactive glass released cobalt ions at biologically relevant concentrations (3-12 ppm) which could activate the HIF-1 $\alpha$ pathway. The alkaline phosphatase (ALP) activity as well as the VEGF gene expression and protein production were significantly enhanced in the cobalteluting composites, demonstrating the advantage of a growth factor-free biomaterial for vascularized bone repair. Another study used $\beta$-tricalcium phosphate (TCP) doped with cobalt (Co-TCP) and investigated the in vitro angiogenic properties with hMSC and HUVECs. The results explained increased VEGF expression by hMSC and enhanced network formation by HUVECs on Co-TCP with respect to undoped TCP [111], suggesting Co ion might have a dual function that mediates the interactions between hMSC and HUVEC for favorable angiogenesis. Recently, dual-ion (silicate and cobalt) delivery cobalt doped silicate microspheres $(\sim 300 \mu \mathrm{m})$ was fabricated as drug-free multifunctional biomaterials, which can synergistically upregulate angiogenesis with the help of silicate ions induced angiogenesis (Detail of silicate ions induced angiogenic effects will be described in next chapter). When $2.5 \mathrm{wt} \%$ cobalt was doped in silicate based microspheres, key angiogenic genes, such as HIF1-a, VEGF and the receptor kinase insert domain receptor (KDR) were synergistically upregulated. In addition, neo-vascular formation was significantly higher in the dual-ion delivered microspheres as evidenced in a chicken chorioallantoic membrane model. This study demonstrated synergistic angiogenic efficacy from dual ions (silicate and cobalt) released from microspheres, and the concept provided here can be extensively applied to repair and regenerate tissues as a growth factor- or drug-free delivery system (Fig. 14D\&E) [112].

\subsubsection{Silicate}

Silicon is an essential element for normal development of bone. Osteoblasts have silicon localized in the mitochondria which plays a major role in the apatite production [113]. There are various silicate-based or -containing biomaterials used for bone, including silica-based bioactive glasses ( $\mathrm{SBGs}$ ), akermanite $\left(\mathrm{Ca}_{2} \mathrm{MgSi}_{2} \mathrm{O}_{7}\right)$, bredigite $\left(\mathrm{Ca}_{2} \mathrm{Mg}\left(\mathrm{SiO}_{4}\right)_{4}\right)$, monocalcium silicate $(\mathrm{CaSiO})_{3}$, dicalcium silicate $\left(\mathrm{Ca}_{2} \mathrm{SiO}_{4}\right)$, tricalcium silicate $\left(\mathrm{Ca}_{3} \mathrm{SiO}_{5}\right)$, silicon-doped calcium phosphates (HA and TCP), silica incorporated calcium phosphate cement (CPC) and silicate-doped calcium carbonate $\left(\mathrm{CaCO}_{3}\right)$, silicon-incorporated glasses and glass ceramics [114]. The

$\mathbf{A}$

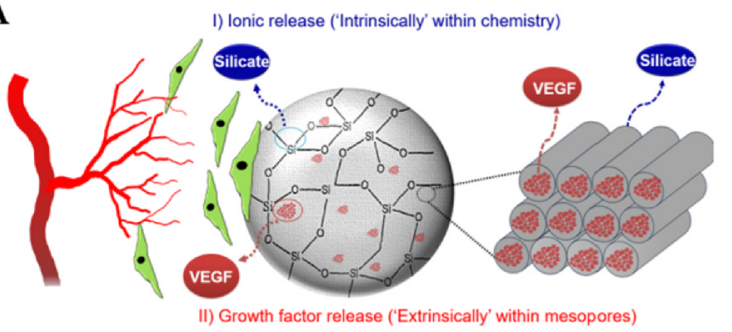

C
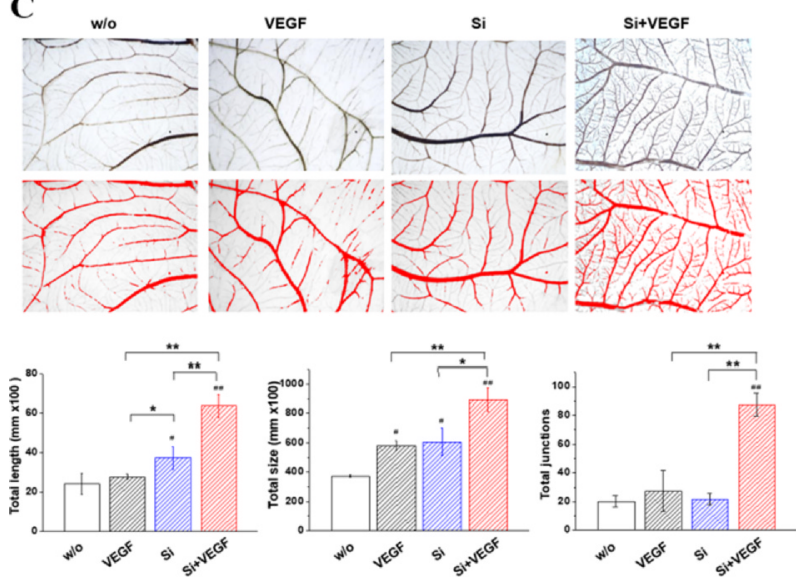

B
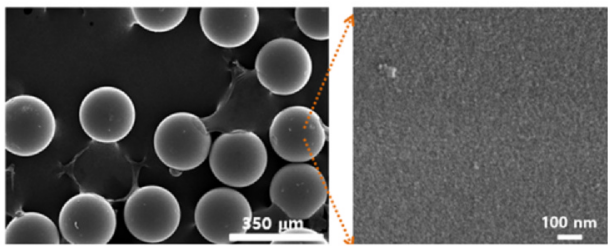

D
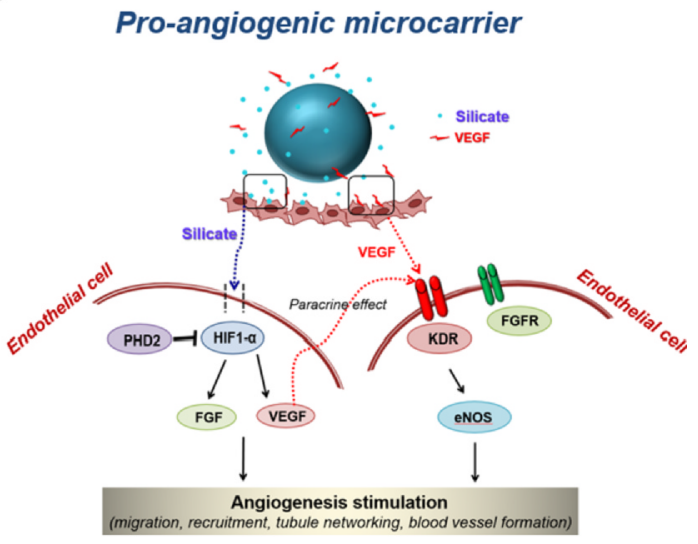

Fig. 15. Pro-angiogenic microcarriers designed to deliver both silicate ions and VEGF. (A) Scheme of co-delivery (silicate ions and VEGF) strategy, (B) mesoporous silica microcarriers, (C) synergistic effect of silicate ion (Si) and VEGF on angiogenesis in CAM model, (D) angiogenic molecular events synergized in the system. Adapted from Dashnyam et al., Biomaterials, 2017 [121]. 
silicate-based biomaterials can release silicate ions during dissolution or cellular degradation which ultimately influences angiogenesis and osteogenesis. Among others, the SBGs have already proven the inherent ability to induce vascular network formation due to the presence and release of silicate ions $[115,116]$. Angiogenesis is an essential event in wound healing, thus bioactive glasses have been widely used for wound repair. They have been shown to accelerate the wound recovery not only in normal but also in diabetic rat model [117]. Bioactive glasses showed an ability to stimulate fibroblasts to release VEGF, bFGF, EGF, collagen I, and fibronectin. Owing to these secreted factors, the fibroblasts quickly moved to the wound area and their differentiation into myofibroblasts was stimulated, by which the production of collagen I and blood vessel formation were enhanced, resulting in accelerated wound healing [118]. The silicate-based biominerals have also been produced into composites or hybrids for bone tissue engineering. Recently, 2D
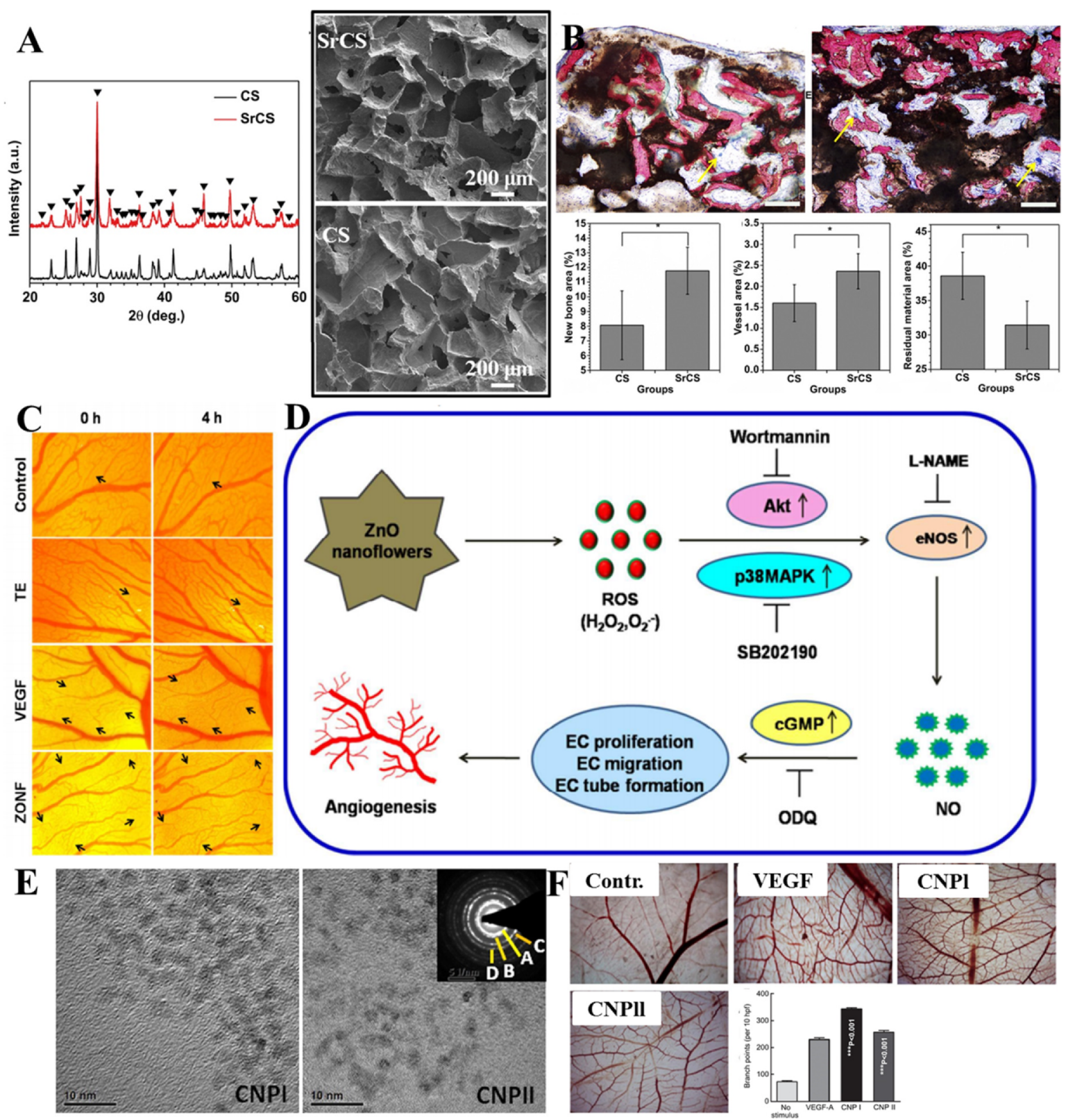

Fig. 16. Proangiogenic role of different kinds of ions ( $\mathrm{Sr}$, Zn, and Ce). (A) After characterization of strontium-doped calcium silicate (SrCS versus Srfree CS) bioceramics by XRD and SEM, (B) the angiogenic role of strontium ions for bone regeneration was proven in osteoporotic critical calvarial defects, revealing excellent angiogenic blood vessels (yellow arrow) in regenerative bone from SrCS. Adapted from Kaili et al., Biomaterials, 2013 [124]. (C \& D) ROS produced by ZnO induced nitric oxide and cGMP-dependent angiogenesis via Akt/MAPK/eNOS pathway, revealed by chicken chorioallantoic membrane study. Adapted from Ayan Kuma et al., J Mater Chem B, 2017 [132]. (E) Cerium oxide nanoparticles (CNPs) varying Ce ${ }^{3+} /$ $\mathrm{Ce}^{4+}$ ratios (CNPl; $\mathrm{Ce}^{3+}$ dominant, CNPll; $\mathrm{Ce}^{4+}$ dominant) accelerated angiogenesis in vivo (especially $\mathrm{Ce}^{3+}$ dominant $\mathrm{CNP}$ ). (F) Vessels formation in using a chicken chorioallantoic membrane model was captured and quantified. Adapted from Soumen et al., Biomaterials, 2012 [139]. 
nanosilicates made of sodium-lithium-magnesium-silicate (Laponite) nanoparticles were embedded in gelatin methacrylate hydrogels and then photocrosslinked to form a composite scaffold [119]. This scaffold promoted ALP activity and mineralization in normal growth medium without any osteoconductive factors. Another study examined the effect of akermanite extract (where silicate ions contained) on receptor activator of nuclear factor-kappa B ligand (RANKL)-induced osteoclastogenesis. The akermanite extracts were shown to suppress osteoclast formation and expression of TRAP, cathepsin K, and NFATc1. Furthermore, angiogenesis and bone formation were dramatically stimulated in a test with ovariectomized rat critical-sized calvarial defect model [120], suggesting the osteoclastic inhibition might contribute to the stimulated osteogenesis and bone formation.

Although many studies have reported the excellent angiogenic ability and the resultant bone formation of silicate-based (silicate ion releasing) biomaterials, the mechanism underlying such events has been elusive. One major reason is that those silicate-based biomaterials release not only silicate ion but also other ions (e.g., calcium, magnesium, or phosphate), making it difficult to decouple the silicate ion effects from the others. Our study aimed to address this issue using mesoporous silica microspheres [121]. The mesoporous silica microspheres were prepared by a sol-gel process to have an average diameter of $\sim 250 \mu \mathrm{m}$ and mesopores. The silica microspheres could release silicate ions slowly with time which significantly stimulated the expression of angiogenic markers (bFGF, KDR, VEGF, eNOS, and bFGFR) of HUVECs (Fig. 15). From a mechanistic study, such an enhancement was proven to be through the activated HIF1 $\alpha$ which was stabilized by the inhibition of prolyl hydroxylase 2 (PHD2). The pro-angiogenic role of silicate ions was further demonstrated to synergize when co-delivered with VEGF, suggesting the promising approach of silicate ion delivery with angiogenic factors. Based on this study, the angiogenic mechanism owing to silicate ions was recently explained [116]. The silicate ion stabilizes HIF1 $\alpha$ (hydroxylation inhibition) by inactivating PHD2, and such an event is thought to share a major trait in common with the case in other metallic ions (e.g., cobalt or copper). Also, silicate ions enhance the production of reactive oxygen species (ROS) and thus affects $\mathrm{Fe}^{2+}$ ions in blocking PHD2. However, more studies are needed to elucidate the precise role of silicate ions by which the PHD2 activity is regulated.

\subsubsection{Other ions or element ( $\mathrm{Sr}, \mathrm{Mg}, \mathrm{Zn}$, Ti, and $\mathrm{Ce}$ )}

There are other minor ions or elements that play a significant role in angiogenesis and osteogenesis. Here, we give some representative examples of the role of strontium, magnesium, zinc, titanium, and cerium ions in angiogenesis and the related tissue healing.

Strontium ion has the ability to stimulate the osteoblast activity, inhibit the osteoclast activity and in general improves bone quality by various intracellular mechanisms $[122,123]$. The role of strontium ions in angiogenesis was proven in rat in vivo studies using strontium-doped calcium silicate bioceramics (Fig. 16A\&B) [124]. The endothelial proliferation and expression of angiogenic gene VEGF increased by strontium ion release. Of note, the efficacy of strontium-CS was demonstrated in osteoporotic critical calvarial defects showing an excellent angiogenic blood supply. Strontium ion has also the ability to indirectly induce angiogenesis by stimulating macrophages to secrete pro-angiogenic factors [125]. In the study, strontium-contained bioactive glass microspheres were shown to polarize macrophages from M1 to M2 phase, and the M2 phenotype released PDGF-BB supporting in vitro and in vivo neo-angiogenesis.

Magnesium has been used in many biomaterials and is known to have osteo-regenerating capacity [126]. An earlier study has reported that high concentration of magnesium ions $(10 \mathrm{mM})$ could modulate endothelial cells in vitro such as endothelial proliferation, secretion of angiogenic factors and synthesis of nitric oxide [127]. Magnesium also plays a vital role in structural and functional integrity of endothelial cells, thereby maintaining vascular homeostasis and preventing atherosclerosis [128]. Like strontium, magnesium ions have also shown to change macrophage phenotype, and thereby indirectly initiate angiogenesis during bone regeneration [129].

Zinc has also the ability to control macrophage phenotype that is responsible for angiogenesis [130]. In fact, zinc in the zinc oxide materials is considered as a potent generator of ROS which plays an important role in inducing angiogenesis through VEGF signaling. For example, electrospun PCL scaffolds incorporating zinc oxide nanoparticles could upregulate proangiogenic genes FGF and VEGF [131]. Another example studied the signaling pathway of ZnO-mediated angiogenesis (Fig. 16C\&D) [132]. It was found that ROS produced by ZnO induced Akt/MAPK/eNOS pathway which further led to production of nitric oxide and cGMP-dependent angiogenesis. Since, zinc is an essential element for osteoblasts functions [133], it is expected that ZnO and other forms of zinc can be a potent ion for both angiogenesis and osteogenesis.

Titanium is essentially used as metallic implants for orthopedic and dental applications. When implanted, titanium metal is constantly exposed to blood and extracellular fluid, leading to leaching of titanium ions in the blood stream and aseptic loosening of orthopedic implants $[134,135]$. Yet, the effect of titanium ions on angiogenesis has been elusive. In one study, titanium dioxide was substituted for $\mathrm{Si}$ in silica-based bioglass. This substitution could induce better angiogenesis, and the Ti ion was proposed as an effective angiogenic promoter [136,137].

Another element that is gaining great interest is cerium owing to its ability to induce angiogenesis by modulating intracellular oxygen environment, leading to protection of endothelial (or progenitor) cells against ROS stress and acceleration of angiogenesis via HIF-1 $\alpha$ activation [138-141]. An important study by Das et al. [139] linked cerium oxide nanoparticles (CNPs) with angiogenesis in vitro and in vivo (Fig. 16E\&F). They established that physicochemical properties (e.g., surface $\mathrm{Ce}^{3+} / \mathrm{Ce}^{4+}$ ratio, surface charge, size, and shape) played a role in modulating angiogenesis. For example, the catalytic activity of CNPs increased with increasing surface area $\mathrm{Ce}^{3+} / \mathrm{Ce}^{4+}$ ratio which ultimately regulating intracellular oxygen level and angiogenesis which yet could not elucidate the direct role of ions released from the nanoparticles in angiogenesis. 


\subsection{Delivery of angiogenic proteins}

During angiogenesis, many signaling molecules are essentially involved, such as VEGF, FGF, hepatocyte growth factor (HGF), platelet-derived growth factor-BB (PDGF-BB), angiopoietin/tyrosine kinase endothelial (ANG/TIE), and transforming growth factor$\beta 1$ (TGF- $\beta 1$ ), which are responsible for inducing and stabilizing the formation of blood vessel networks. The controlled and targeted delivery of these angiogenic molecules has been researched for therapeutic cure of tissue damages and diseases such as wound healing and ischemia. Each angiogenic molecule binds to specific receptors on the cells to elicit the necessary biological response. VEGF and its isoforms bind to receptors VEGFR-1 and VEGFR-2, while bFGF binds to receptors FGFR1b/c, FGFR2b/c, FGFR3b/c and FGFR4. Further, PDGF binds to receptors PDGFR $\alpha$ and $\beta$, Ang-1 to Tie-2 receptor, and Ephrin to Ephrin receptor tyrosine kinase [142].

For favorable therapeutic effects, a continuous delivery of these biomolecules over a period of weeks or months is essential. Thus, biomaterials are often developed to enable the controlled and sustained release of the angiogenic molecules. Various types of natural and synthetic scaffolds were used as delivery vehicles including hydrogels, micro-/nano-porous scaffolds, and micro-/nano-particles $[143,144]$. The pore architecture of scaffolds is one of the key factors that determines the diffusion of angiogenic factors and consequently cell survival and migration, and nutrient delivery [26,145]. Particles are useful to encapsulate angiogenic factors and to release them slowly through a matrix. Hydrogels can incorporate angiogenic factors in physically or chemically crosslinked networks and release them in a controlled manner [146]. Furthermore, vascular supportive structures micro-engineered are beneficial for functional and metabolic integration of the construct in host tissues with minimal use of biofactors and modification of host injury sites $[95,147]$. Some of the most important angiogenic molecules and their delivery strategies through biomaterials are explained below.

\subsubsection{VEGF delivery}

VEGF family consists of various members such as VEGF-A, -B, -C, -D, -E, -F [148,149] and placental growth factor (PlGF) [150]. In this family, VEGF-A and its isoforms play a major role in angiogenesis while PIGF is important for homeostasis [151,152]. Hypoxia upregulates VEGF-A expression which induces osteoblast formation by binding to the RANKL (receptor activator of nuclear factorkappa B ligand) [153]. The reason for VEGF-A upregulation is that during hypoxic conditions, HIF-1 $\alpha$ regulating von Hippel-Lindau tumor suppresser is induced [154], and this gene binds to hypoxia responsive element leading to VEGF-A upregulation. PlGF has not shown any direct effect on ECs but is known to enhance the effect of angiogenic factors by inducing cell membrane receptor VEGFR-2
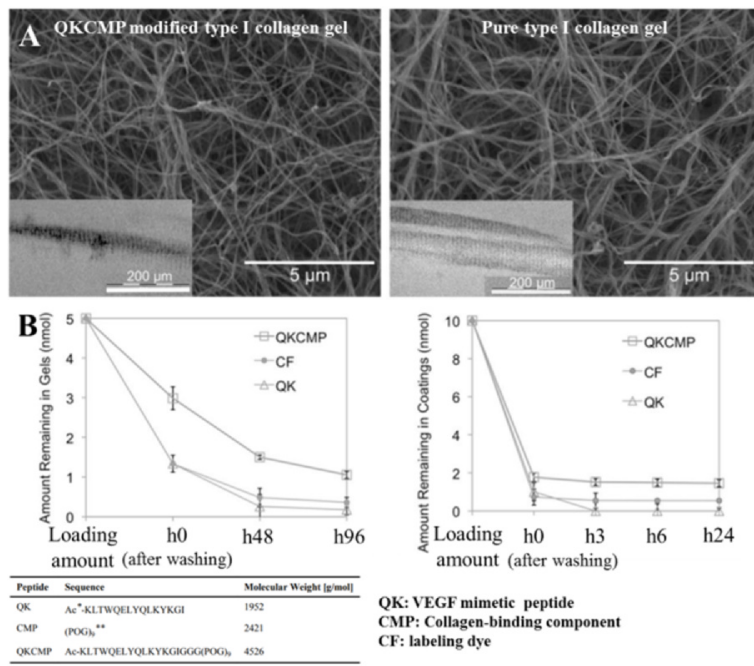

QK: VEGF mimetic peptide

CMP: Collagen-binding component

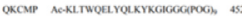
CF: labeling dye

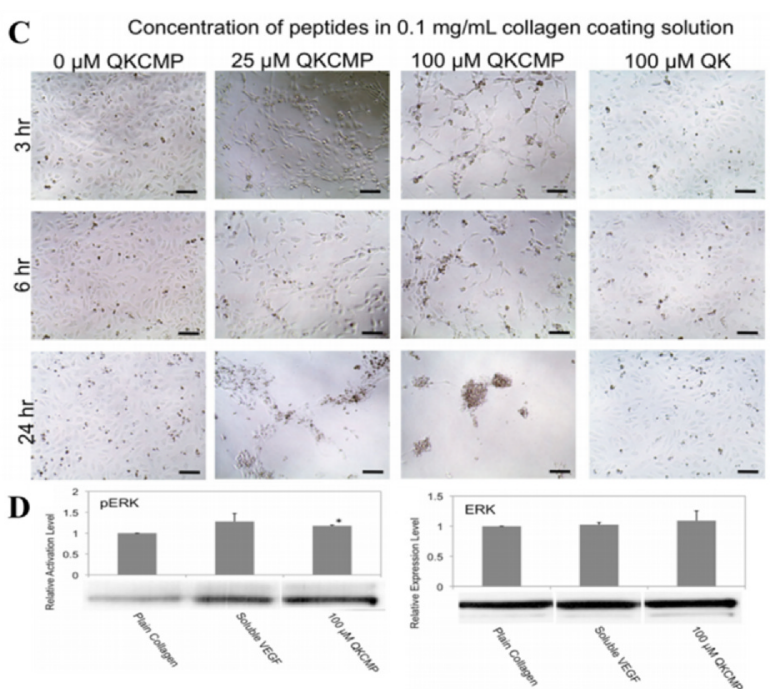

Fig. 17. VEGF mimetic peptide QKCMP engineered on collagen type 1 as matrix-bound growth factor for directing angiogenic cues. Collagenbinding component (CMP) was added to a VEGF mimetic QK domain to prepare QKCMP, which capable of binding type I collagen and giving biological effects on endothelial cells. (A) Scanning electron and transmission electron micrographs (insets) of QKCMP modified type I collagen gel (left) and pure type I collagen gel (right), suggesting that the presence of QKCMP did not interfere with collagen fiber formation. (B) Cumulative release profiles of CF-labeled QKCMP, CF-labeled-QK, and CF itself from type I collagen gels (left) and type I collagen coatings (right), showing that the angiogenic peptide (CMP domain of QKCMP) can bind to a collagen matrix with high affinity without adopting a covalent approach and can turn a soluble VEGF mimetic QK molecule for giving angiogenic functionality. (C) When ECs (HUVECs) were cultured on the QKCMP-immobilized collagen (25 and $100 \mu \mathrm{M}$ of QKCMP in $0.1 \mathrm{mg} / \mathrm{mL}$ collagen coating solution), ECs exhibited network morphology, while comparatively, HUVECs plated on plain collagen coating displayed only cobble stone morphology. This indicated that QKCMP, immobilized to the collagen substrate through the CMP domain, is able to elicit a unique EC response. (D) Extracellular signal-regulated kinase 1 and 2 (ERK1/2) was investigated due to its crucial role in VEGF dependent activation of ECs. In comparison to cells grown on plain collagen substrate, cells seeded on QKCMP immobilized collagen showed a marked increase in phosphorylation level, while maintaining the same level of ERK1/2 expression as evidenced by Western blot. Adapted from Chan et al., Advanced Functional Materials, 2011 [168]. 
[155]. Another family member of VEGF, VEGF-B plays an initial inflammatory role in bone formation as evidenced in a bone fracture experiment using knockout mice $v e g b^{+/+}$and wild type $v e g f b^{-/-}$. The in vivo model showed that VEGF-B level increased significantly during initial inflammation period [156]. Another isoform, VEGF-C plays a role in antigen presenting selection, allo-immunity and lymphangiogenesis, inducing vascular permeability, anti-tumor activity, and selective maturation of cells [157,158]. VEGF-C is therefore instrumental in keeping metastasis and homeostasis as shown by a study on mouse cornea [159]. Further, VEGF-D is expressed in late phase of osteogenesis activating the VEGFR-3 receptor, thereby, paving the way for osteoblast maturation and differentiation [160]. Studies on human osteoblasts have shown that VEGF-D induced mineralized nodule formation and higher osteocalcin induction after 14 days of treatment [160]. To incorporate the family of VEGF family molecules, various scaffolds have been fabricated. For example, Kaigler et al. [161] used poly(lactic-co-glycolic acid) (PLGA) scaffold incorporated with VEGF and tested the efficacy in a rat calvarium defect model. Results showed a two-fold increase in the blood vessel formation with enhanced total bone mineral density by the VEGF delivery. Presenting VEGF in a prolonged localized form has the potential to enhance bone formation. With this aim, the VEGF-releasing polymeric scaffolds with bioactive glass coating was studied in vitro and in vivo in a rat critical-sized defect model [162]. The releasing VEGF and the bioactive glass coating were shown to enhance the angiogenesis and bone maturation.

In the above studies, the VEGF was physically trapped in the scaffold and were subsequently released to promote neovascularization. Covalent immobilization of angiogenic factors onto scaffolds is another approach to improve pro-angiogenic cell signaling [163]. The main advantage of covalent immobilization of biomolecules is that the method can sustain signal transduction for a long period. Chen et al. [164] showed that ECs responded differently to free and matrix-bound VEGF. Chiu and Radisic [165] covalently linked VEGF (and/or Ang1) onto collagen scaffolds and observed increased EC density and tube formation in vitro with increased blood vessel density in vivo. Researchers also used synthetic VEGF-mimetic peptide to covalently link with a hydrogel structure for better cell-receptor interactions. As a representative example, a synthetic VEGF-mimetic peptide QK mimicking the 17-25 helix region of VEGF was prepared by D'Andrea et al. [166]. This peptide QK was also differently modified to link with PEG hydrogels $[167,168]$. Further, RGD sequence was also incorporated into the hydrogels to promote angiogenesis. When the peptide modified hydrogel was implanted in a mouse cornea micropocket, vascular parameters including branch points, vessel density, fractal dimension, and lacunarity parameter significantly increased when compared to VEGF alone or VEGF-incorporated hydrogels. To advance the system, Chan et al. [168] added collagen-binding component (CMP) to QK to prepare QKCMP (Fig. 17). Thus, the angiogenic peptide can bind to a collagen matrix with high affinity without adopting a covalent approach. When ECs were cultured on the QKCMP-immobilized collagen, multiple filopodia protrusions were developed in the cellular networks, and the cell morphogenesis was shown to alter by spatially controlling the QKCMP immobilization. The studies suggest that the matrix-bound molecules, not their soluble form, may be effective in VEGF signaling of ECs.

\subsubsection{PDGF delivery}

PDGF (platelet-derived growth factor) is the major growth factor released from stimulated platelets and is mitogenic for fibroblasts, osteoblasts and smooth muscle cells [169]. The PDGF family (PDGF-A, -B, -C, and -D) has been found to stimulate the osteoblast migration by chemotactic factors, thereby enhancing proliferation and calcium mobilization [170]. One of its family members, PDGF-CC was successful in enhancing postischemic revascularization of the heart and limb. PDGF-CC not only revascularizes ischemic sites by mobilizing EPCs to the site but also induces the differentiation of bone marrow cells into smooth muscle cells [171]. Another homodimer, PDGF-BB plays a vital role in osteogenesis during bone modelling and remodeling [172]. In the study, improved angiogenesis and osteogenesis occurred owing to the secretion of PDGF-BB by preosteoclasts which led to recruitment of EPCs and MSCs, underscoring the increase of PDGF-BB secretion may be a novel therapeutic strategy.

The above concept was used to recruit MSCs using scaffolds. PDGF-B adenovirus was integrated with mesoporous bioglass-silk fibrin scaffolds [173]. Following a subcutaneous implantation in mice, the scaffolds were shown to release PDGF-B adenovirus for up to 3 weeks, and then repaired the osseous calvarial defect by recruiting host MSCs to the defect site. The delivery of PDGF was also possible by the mesoporous silica particles [174], where silver was incorporated for antibacterial actions. The multifunctional scaffolds promoted MSC osteogenesis and vascularization along with bactericidal property enabled by silver ions. Like VEGF, PDGF was also immobilized and its effect on the formation of microvasculature was studied. The solubility of PDGF-BB is rapid in vivo, hence for a sustained signaling the solubility must be slowed. To achieve this, PDGF-BB was covalently linked to PEG hydrogel and HUVECs were seeded onto the hydrogel surface [175]. The HUVECs formed tubules with extensive networks and branching. The study further examined the difference between soluble PDGF-BB and covalently immobilized PEG-PDGF-BB in a mouse cornea angiogenesis assay. The PEG/PDGF-BB showed increased vessel density when compared with the case with soluble PDGF-BB.

\subsection{3. $b F G F$ delivery}

FGF is a large family of polypeptides that has diverse roles in cell proliferation, migration, angiogenesis and differentiation [176]. FGF has been widely used in tissue engineering and regenerative medicine owing to its potent biological activity [177]. Basic FGF (bFGF or FGF2) was originally derived from pituitary gland. The angiogenic character of bFGF was well demonstrated [178] in terms of stimulating EC migration, invasion, and production of plasminogen activator in vivo. This endothelial stimulation by bFGF could produce VEGF through autocrine and paracrine mechanisms [179]. As far as bone is concerned, bFGF plays an important role in the bone homeostasis and fracture repair [180-182]. For example, Kigami et al. [183] studied the effects of bFGF on angiogenesis and bone regeneration in rat calvarial noncritical-sized bone defects. They used an absorbable collagen sponge with or without bFGF and implanted in bone defects. After 28 days of implantation, the sponge with $0.3 \%$ bFGF (the highest concentration used) formed new bone with the largest area $(85.2 \pm 0.9 \%)$ and blood vessels $(47 \pm 25 \%)$. In a recent study, the effect of bFGF-containing HA/ 
collagen composite on prefabricated vascularized allogenic bone graft was investigated [184]. Bone formation and angiogenesis were stimulated by vascular bundles or bFGF-containing HA/collagen composites. The in vivo study showed the bone formation with high angiogenic activity which however lacked bone resorption. The bFGF was also engineered to be over-expressed in MSCs to accelerate fracture healing [185]. When the bFGF over-expressing MSCs were injected around the fracture site, the healing process was quickly accelerated via stimulated angiogenesis and differentiation of MSCs to bone-producing osteoblasts. The release of bFGF was also shown to be highly effective in mural cell recruitment which is important for the vasculature maturation. Chu et al., designed an injectable polyvalent coacervate of polycation, heparin, and bFGF [186,187]. The bFGF released from the coacervate was more effective in chemotaxis of pericytes than free bFGF. Mural cells were shown to participate actively in the whole angiogenesis process, and the pericytes were recruited to close proximity of the ECs within 7 days of injection in vivo.

In the previous studies, there was no mechanism to control the release of bFGF from the scaffolds or the cells. However, for clinical translation, the payload must be released in a controlled fashion to achieve the dual aim of safety and efficacy. To achieve this, acoustically-responsive scaffolds were developed to deliver growth factors spatiotemporally [186,187]. Fibrin scaffolds were loaded with bFGF-containing sonosensitive emulsion where the bFGF could be released by the ultrasound. When the scaffolds were implanted subcutaneously, the bFGF-releasing scaffolds showed great perfusion ( 3.3 fold) and blood vessel density (1.7 fold) under ultrasound exposure. This scaffold is considered to offer a new stimuli-responsive delivery platform for growth factors that can find diverse applications including on-demand vascularization in bone fractures.

\subsubsection{TGF- $\beta 1$ delivery}

TGF- $\beta$ superfamily of genes has over 30 members including three TGF- $\beta$ isoforms, activins, and BMPs, and controls important developmental and homeostatic processes $[188,189]$. TGF- $\beta$ is the most widely studied isoform that has great implication in chondrogenesis [190] and plays a main role during bone development and growth, and in repair of fracture callus [191]. Immediately after fracture, the TGF- $\beta 1$ levels increased tremendously to differentiate bone stem cells to osteogenic lineage. An in vitro study with MSCs showed that the osteogenic differentiation by TGF- $\beta 1$ was mediated by TAZ (PDZ-binding motif) which increased the osteogenic-specific runt-related transcription factor 2 (RUNX2)-dependent gene transcription [192]. TGF- $\beta$ also plays an important role in vasculogenesis and angiogenesis in a dose-dependent manner [193]. Therefore, a careful dose selection of TGF- $\beta 1$ is needed to improve bone angiogenesis for tissue repair.

In one of the earliest studies, gelatin hydrogel incorporating TGF- $\beta 1$ was implanted into a skull defect of rabbit, and the resultant bone mineral density was measured at 8 weeks [194]. The mineral density in the hydrogels with TGF- $\beta 1$ was observed to significantly increase with respect to those without TGF- $\beta 1$ ( $\left.84.3 \mathrm{vs.} 64.9 \mathrm{mg} / \mathrm{cm}^{2}\right)$. The use of TGF- $\beta 1$ with BMP2 was also shown to improve bone repair. For this, DermaMatrix ${ }^{\mathrm{TM}}$ with either of the four combinations (vehicle, 350 ng BMP2, 200 ng TGF- $\beta 1$, or 350 ng BMP2 + 200 ng TGF- $\beta 1$ ) was inserted into suturectomy sites of rabbits [195]. Bone tissues resembling native bone were produced in
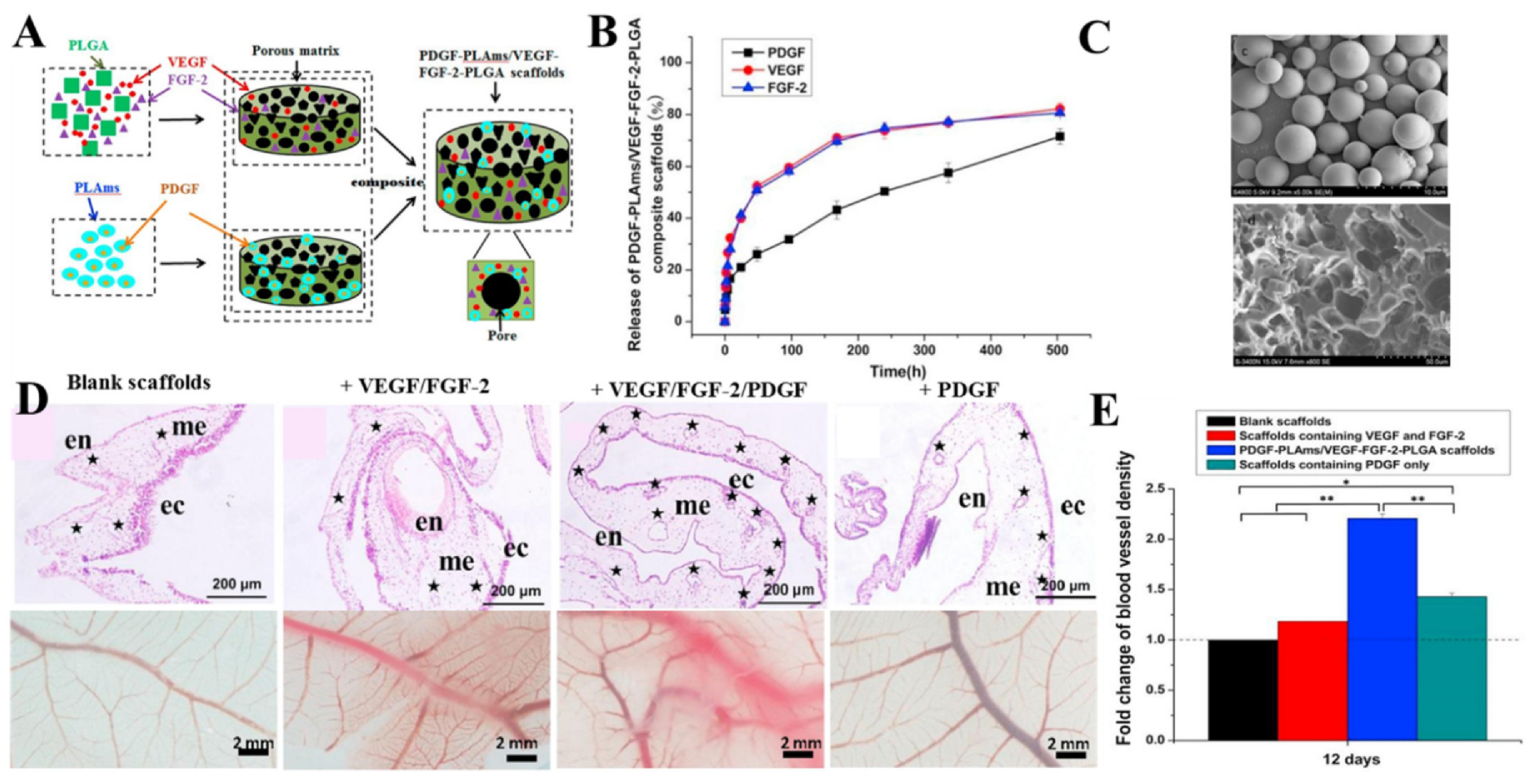

Fig. 18. Triple growth factors delivery for angiogenesis. (A) Three growth factors (VEGF, FGF-2, and PDGF) were incorporated together into single scaffold. The VEGF and FGF-2 were incorporated in PLGA scaffolds, while the PDGF was incorporated in PLA-microsphere (PLAms) which was further encapsulated into PLGA scaffolds. (B) Independent controlled release of the three growth factors was achieved for $\sim 500 \mathrm{~h}$. (C) SEM images of PDGF-loaded PLAms (up) and porous PLGA scaffold (donw). (D) Angiogenic effects of triple delivery of VEGF, FGF-2 and PDGF from scaffolds from the CAM model, resulting in a dramatic increase in the number of mature blood vessels in the mesoderm. (E) Histological response of CAM was quantified. en: endodermal, ec: ectodermal, me: mesoderm, star: blood vessels. Data expressed as mean $\pm \mathrm{SD}(\mathrm{n}=3$; ** $\mathrm{P}<0.01 ; * \mathrm{P}<0.05)$. Adapted from Yan et al., Cellular Immunology, 2017 [199]. 
TGF- $\beta 1$ group, which was ascribed to the native intramembranous ossification pathway. The TGF- $\beta 1$ was also delivered in combination with VEGF through mesoporous calcium phosphates which enabled slow release of growth factor due to the high surface charge of calcium phosphates [196]. Furthermore, TGF- $\beta 1$ was delivered controllably through starPEG-heparin hydrogel where enzymatically cleavable peptide was conjugated with TGF- $\beta$ [197]. In wound healing model, the hydrogels effectively released TGF$\beta$, supporting dermal fibroblast proliferation and differentiation to myofibroblasts, suggesting the hydrogels can be bioactive wound dressings. One intriguing study used the approach of TGF- $\beta$ receptor recruitment to cilia in osteoblasts. For this, tricalcium phosphate was designed with varying surface topographies (grain size $=3.4 \mu \mathrm{m}$ or $0.9 \mu \mathrm{m}$ ) to regulate ciliary recruitment of TGF receptor [198]. The submicron-scale affected the morphology, primary cilia expression and recruitment of p-TGF- $\beta$ R II into the cilium of MSCs. At 12-week of intramuscular implantation in dogs the submicron scale induced bone formation whereas the microscale showed no significant bone formation. This approach is unique to control the biomaterial-driven TGF- $\beta$ recruitment that can dictate cell morphology and bone formation.

\subsection{Combinatory and sequential delivery}

The combinatory and sequential delivery of angiogenic molecules with controlled dose and release rate will help in augmenting and synergizing the actions of each factor in angiogenesis. Recently, scaffolds that sequentially release a mixture of angiogenic growth factors (VEGF, FGF-2, and PDGF) were developed (Fig. 18) [199]. To achieve this, the VEGF and FGF-2 were incorporated in PLGA scaffolds, while the PDGF was incorporated in PLA microspheres which was further encapsulated into PLGA scaffolds. The results showed the VEGF and FGF-2 released more than PDFG during the first 7 days, while the PDGF release became significantly higher after 7 days. Due to the sequential action of VEGF/FGF-2 and PDGF the tubular formation of HUVECs in vitro and the blood vessel maturity in vivo in CAM could be significantly promoted. The combination of Si ion with VEGF in a microsphere has recently demonstrated synergized actions in angiogenesis [121]. The mesoporosity of silica allowed the loading of VEGF and releasing over a couple of weeks while Si ions intrinsically present were leached slowly at therapeutically relevant levels (few ppm per day). The dual release of silicate and VEGF from the silica microspheres significantly enhanced the migration, chemotactic homing and tubulogenesis of ECs, and the neovessel formation in CAM model in vivo, demonstrating the synergistic actions of ion and growth factor in angiogenesis and thus suggesting a potential co-delivery platform for vascularized bone tissues.

The angiogenic factors are often administered together with osteogenic factors, such as BMP2 for bone regeneration purposes. The angiogenic process is relatively an early event, which is then followed by osteogenesis and matrix maturation, therefore, the sequential delivery of angiogenic/osteogenic factors through biomaterials is a promising approach to accelerate bone regeneration.
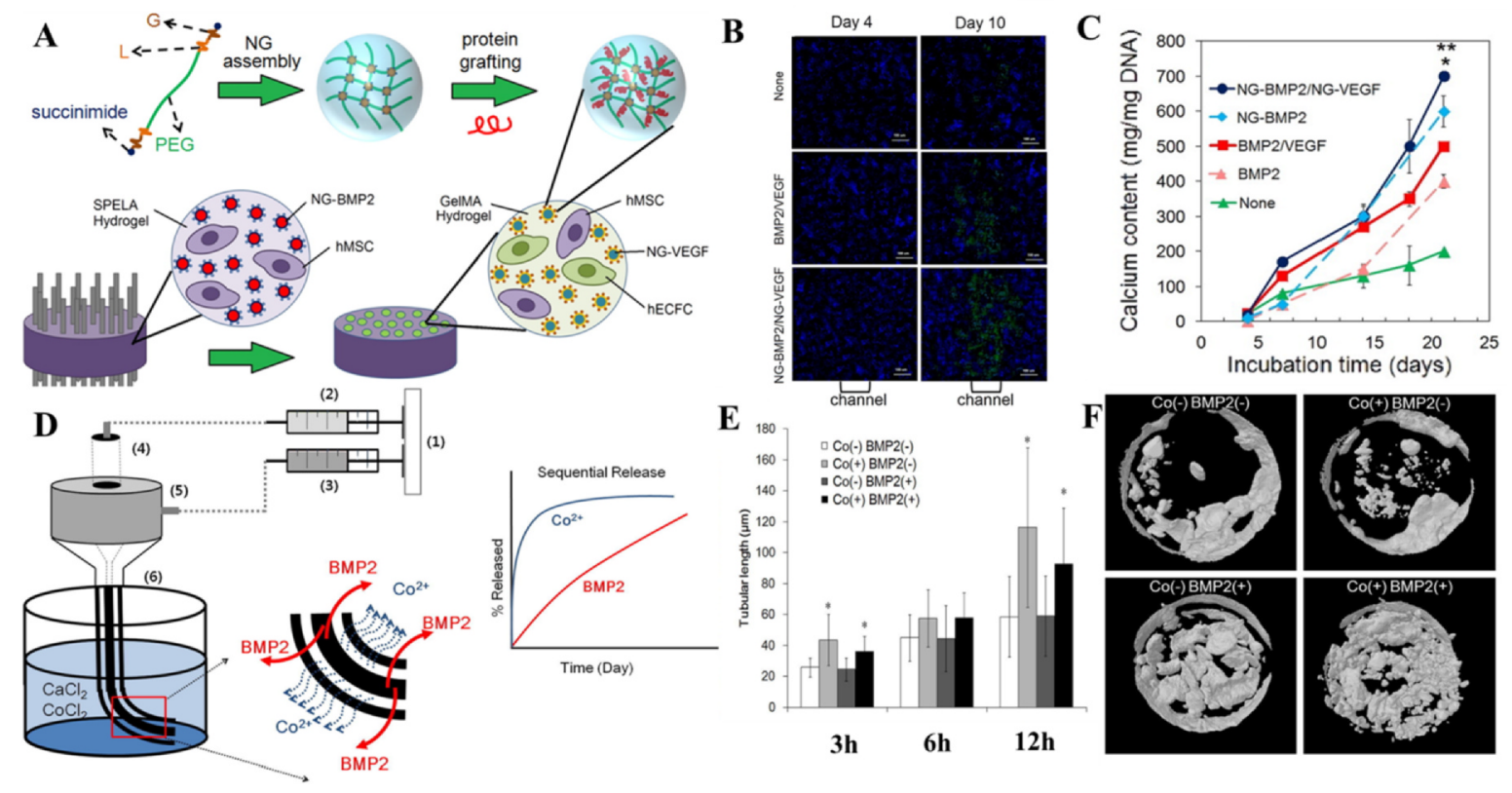

Fig. 19. Combinatory and sequential delivery of angiogenic factors (dual growth factors or growth factor with ion). (A) Schematic diagram for GelMA microchannels, grafting dual growth factors, in osteogenic SPELA gel. (B) Timed-release of VEGF in the microchannels in 10 days from NG(10) and BMP2 in the matrix in 21 days from NG(21) resulted in highest extent of (C) vasculogenic (CD31 (green) and dapi (blue)) and (D) osteogenic differentiation of the encapsulated endothelial colony-forming cells and hMSCs compared to direct addition of VEGF and BMP2. Adapted from Danial et al., J Control Res, 2016 [205]. (D) Hydrogel fibrous scaffolds comprised of a collagen-based core, loading BMP2, and an alginate-based shell, incorporating angiogenic Co ions, were constructed. Time-released Co ions as an angiogenic element together with the osteogenic factor BMP2 from scaffolds synergistically lead (E) increase of tubular formations and (F) in vivo bone regeneration compared to controls. Adapted from Perez et al., Acta Biomater, 2015 [206]. 
Many studies have been carried out for this purpose, and the combinations of biomaterials developed can be categorized mainly into two: i) hydrogels or cements or scaffolds that incorporate microparticles or nanoparticles, and ii) core-shell designs that can separate the delivery factors. Some representative examples are calcium phosphate cement incorporating alginate microspheres to sequentially release PDGF and BMP-2 [200], core-shell electrospun PCL fiber incorporating mesoporous bioactive glass nanospheres for sequential release of FGF-2 and FGF-18 [201], PLGA nanoparticles embedded in alginate microcapsules for dual delivery of VEGF and BMP-2 [202], polypropylene fumarate scaffold with gelatin microspheres for VEGF/BMP-2 delivery [203], and HA/PCL scaffolds for the delivery of VEGF-165 and BMP-2 [204].

Among those, the PEG nanogels developed for a spatiotemporal release of VEGF followed by BMP-2 demonstrated well the stimulated vasculogenic and osteogenic differentiation (Fig. 19A-C) [205]. In the study, patterned nanogels (NGs) were tailored to release grafted VEGF in 10 days and BMP-2 in 21 days by changing the molecular weight and segment (lactide and glycolide) length of PEG. The enhanced vasculogenic and osteogenic differentiation by the sequentially released VEGF and BMP-2 was also demonstrated to be mediated by the paracrine signaling factors. A recent study also used angiogenic ion cobalt in the co-delivery with BMP2. Collagen/alginate core-shell hydrogels were used to deliver cobalt ion and BMP-2 in a sequential manner (Fig. 19D-F) [206]. The sequential delivery system demonstrated the angiogenic effect of cobalt ion and the synergistic role of BMP-2/cobalt in bone formation in vivo. Not only protein forms, but the plasmid form of VEGF and BMP-2 was also delivered through collagen-nanohydroxyapatite scaffolds by coupling polyethyleneimine [207]. The dual gene-activating scaffolds demonstrated superior MSC-mediated osteogenesis in vitro and increased vascularization and bone repair in vivo. Similarly, a hydrogel scaffold incorporating chitosan nanoparticles with BMP-2/VEGF plasmid was developed as a gene-activating scaffold [208]. The scaffold implanted in critical-sized
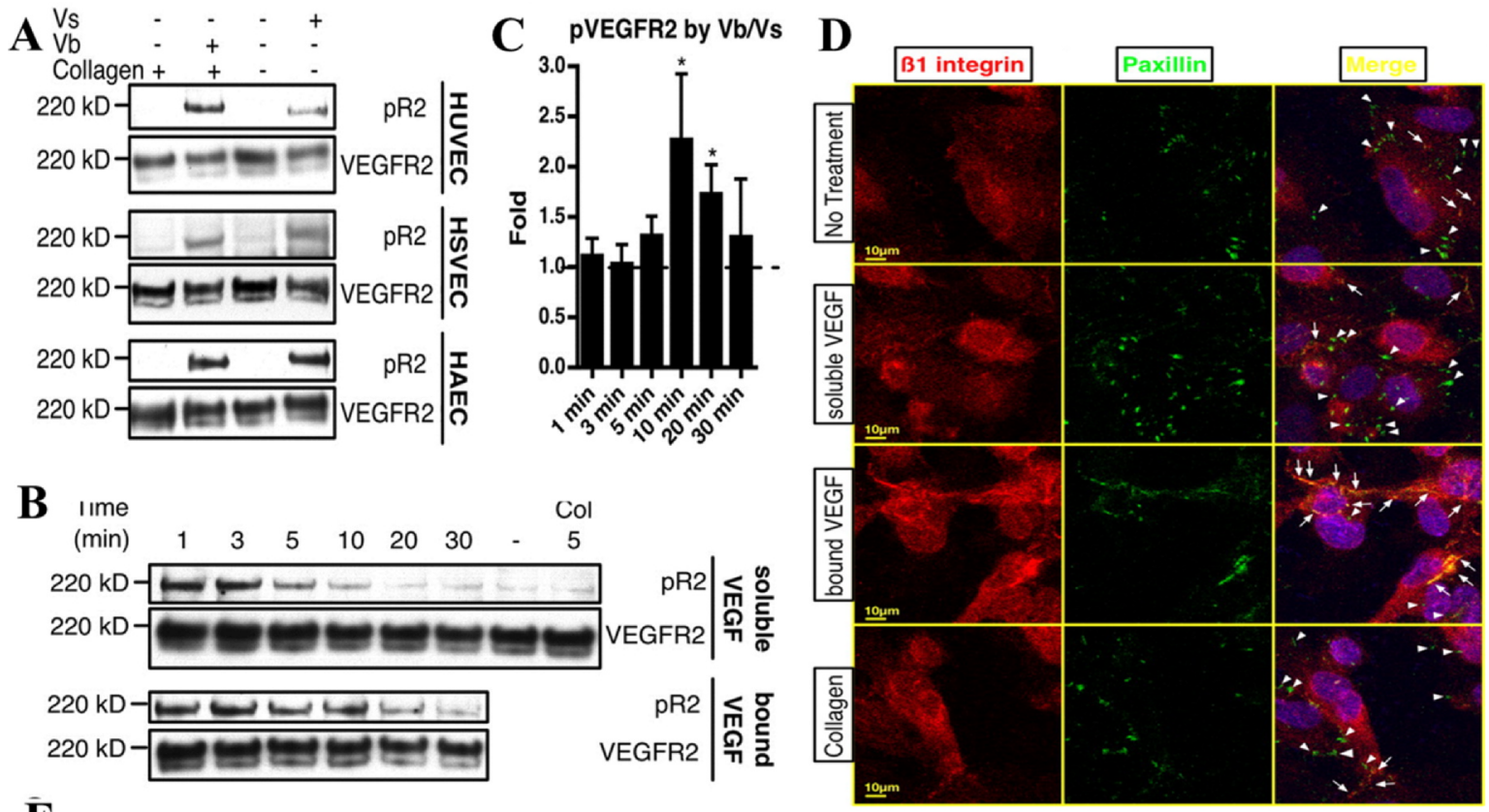

$\mathbf{E}$

Soluble VEGF
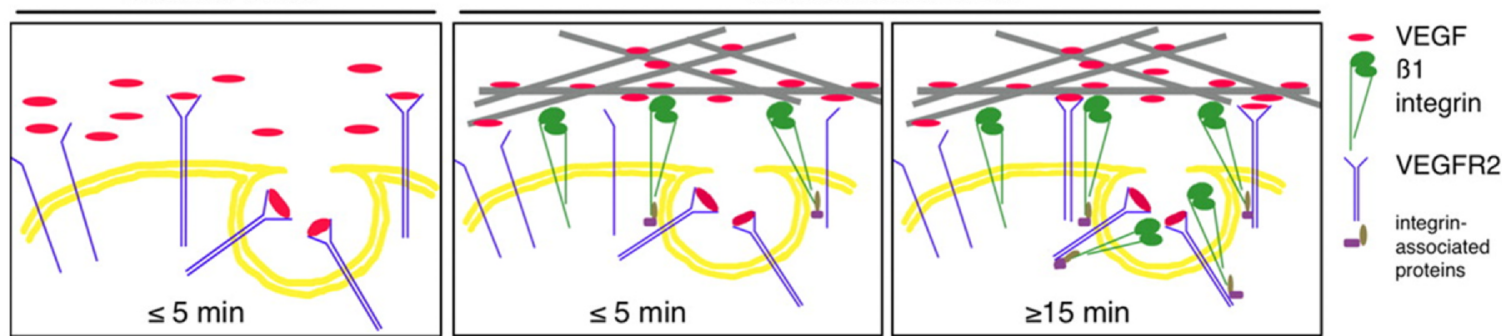

Fig. 20. Presentations of angiogenic factors through affinity-driven matrix-bound approach. Matrix-bound VEGF can elicit better VEGF-receptor interaction compared to free soluble VEGF. We found that matrix-bound VEGF165 (Vb) remained more active by evaluating its ability to phosphorylate VEGFR2 when compared with soluble form (Vs) at identical concentrations for 5 min using multiple endothelial cell types. (B\&C) Exposure of VEGF165 to ECs revealed prolonged activation of VEGFR2 only when the growth factor was bound to matrix (*, $\mathrm{P}<0.05$ between Vb and Vs). (D) $\beta 1$ integrin redistributes to focal adhesion upon stimulation by matrix-bound VEGF165. $\beta 1$ integrin (red), paxillin (green), and TOPRO3 (nucleus, blue). Arrows indicate colocalization of $\beta 1$ integrin and paxillin, arrowheads show focal adhesions without $\beta 1$ integrin-positive staining. (E) A model in which matrix-bound VEGF progressively facilitates proximity and subsequent binding to $\beta 1$ integrins was proposed. This interaction mediates receptor clustering and redistribution of $\beta 1$ integrins to focal adhesions. Adapted from Chen et al., Journal of Cell Biology, 2010 [164] 
bone defects induced significantly higher bone volume, which resulted from the VEGF-activated blood vessel formation during the early stage in fracture repair.

\subsection{Matrix-bound presentation of angiogenic factors}

As witnessed above, a major focus on the biomaterials-aided manipulation of angiogenic factors is to release them slowly to cells. However, some recent studies have highlighted that when the growth factors are bound properly to a matrix they can function even better than the soluble form released [164] (Fig. 20).
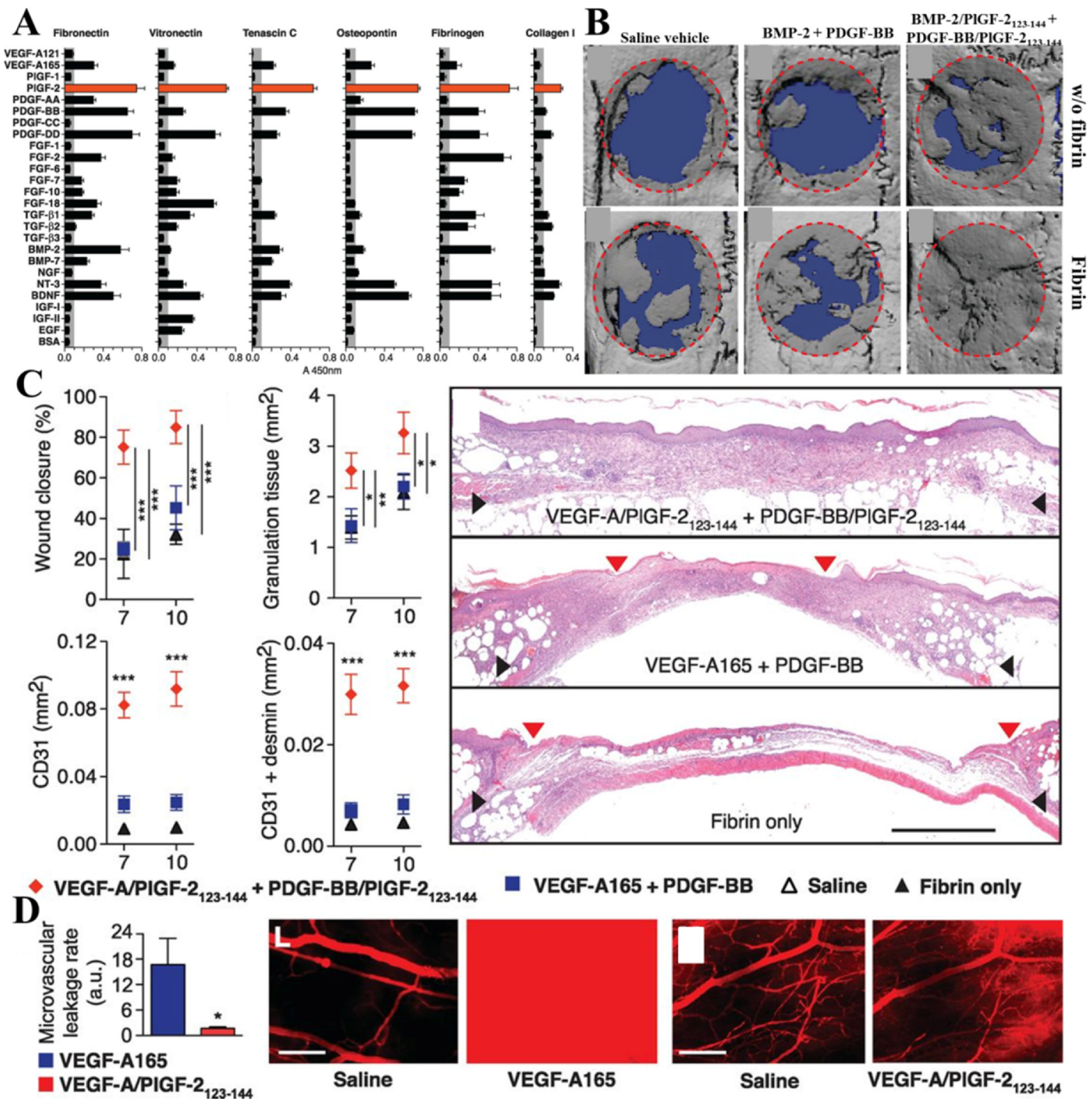

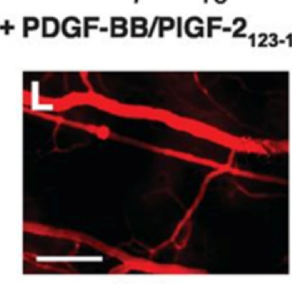

Saline

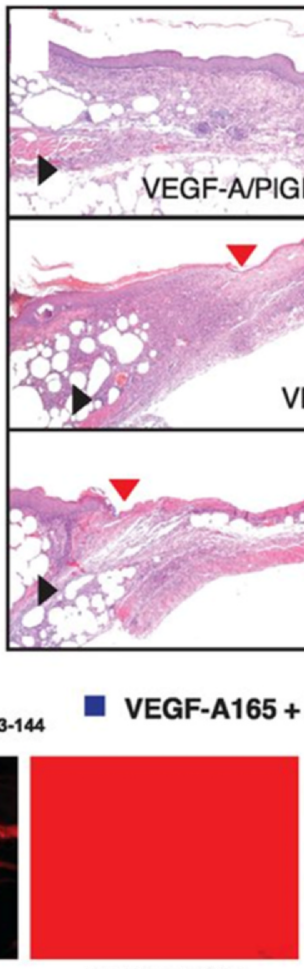

VEGF-A165

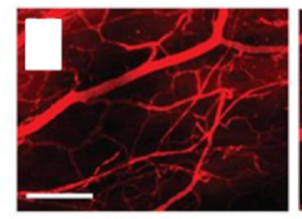

Saline
Fibrin only

Fig. 21. Growth factors engineered for super-affinity with PlGF-2 $2123-144$ to ECM accelerate soft/hard tissue healing via modulation of angiogenic receptor. (A) Numerous growth factors (GFs) are screened to investigate binding affinity to myriad matrix. Among all the GFs screened, PlGF-2 displayed the strongest binding to all of the ECM proteins tested. (B) Fusion of PDGF-BB/PlGF- $2_{123-144}+$ BMP-2/PlGF- $2_{123-144}$ with fibrin and implanting into rat calvarial defect produced $100 \%$ coverage after 4 weeks. (C) Low doses (200 ng) of VEGF-A/PIGF-2 $123-144$ and PDGF-BB/PlGF$2_{123-144}$ in fibrin matrix promoted skin wound healing in full-thickness back-skin wounds model (6 mm in diameter) using diabetic mice, whereas wild-type GFs did not. Biomolecules were delivered in a fibrin matrix once. (D) VEGF-A/PIGF-2 ${ }_{123-144}$ induces much less vascular permeability than the same dose of wild-type VEGF-A165 (500 ng) using a model of vessels leakage in the skin of the mouse ear. When VEGF-A/PIGF-2 $123-144$ was injected, only $10 \%$ of leakage occured compared to wild-type VEGF-A165, giving possibility of solving a major problem for clinical use of VEGF-A. Adapted from Martino et al., Science, 2014 [210]. 
A $\uparrow$ Angiogenesis $\quad \uparrow$ Axonogenesis $\quad \downarrow$ Physical Barrier

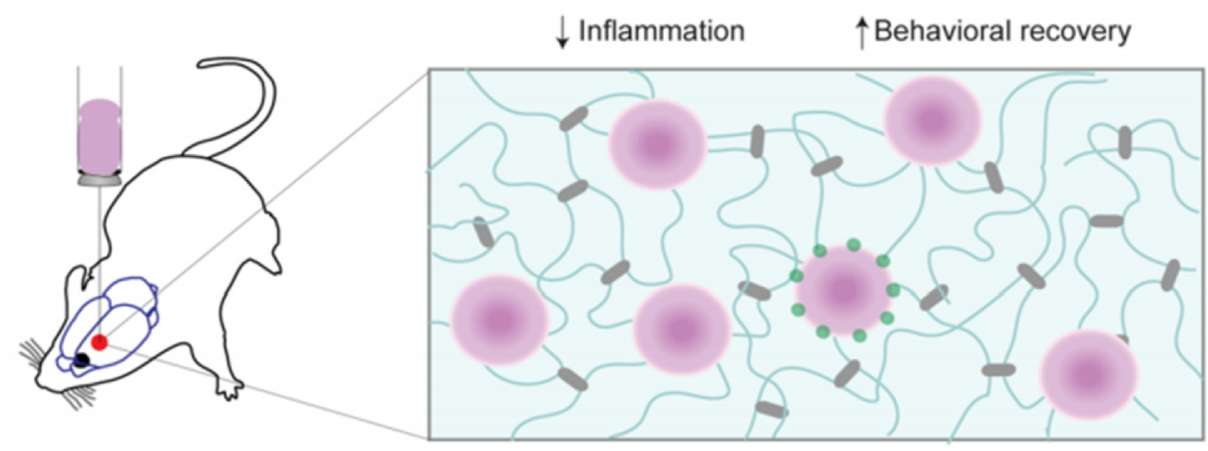

Stroke

HA polymer
Crosslinker

$\circ$ ○ soluble VEGF
Heparin nanoparticles $(\mathrm{nH})$

hcV (Heparin particles + high cluster VEGF)
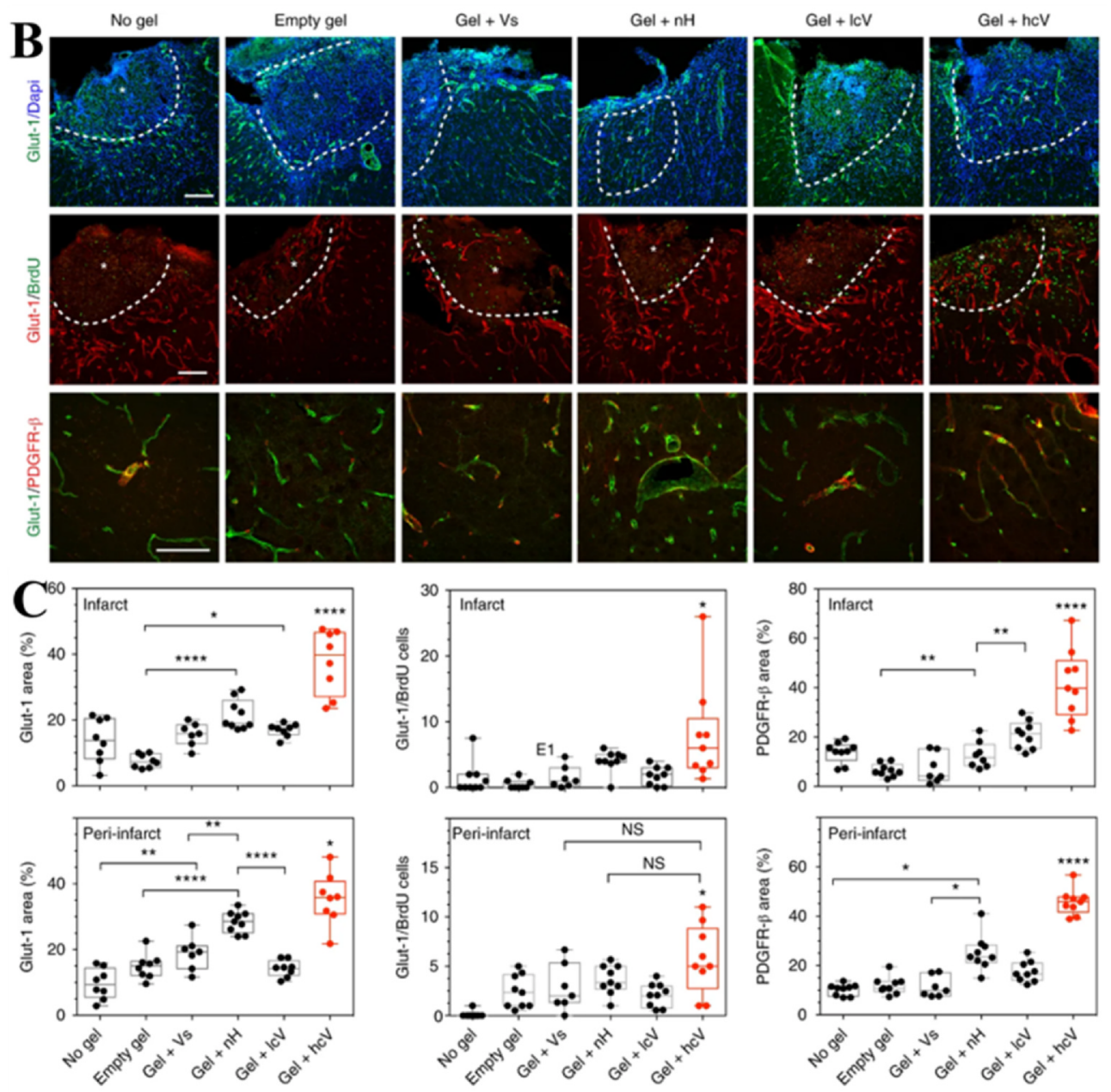

(caption on next page) 
When growth factors are covalently coupled to a biomaterial, their release will mostly depend on the biomaterial degradation. To exploit the degradation-mediated growth factor release, angiogenic factors and biomaterials were engineered to incorporate proteasesensitive sites [209]. One landmark study by Hubbell and colleagues has utilized the affinity binding approach of a matrix using placenta growth factor (PlGF), which, being screened from a variety of ECM proteins, was shown to have an exceptional affinity across various kinds of growth factors, including VEGF [210] (Fig. 21). Specifically, the domain PlGF-2 $123-144$ with heparin binding sequence can bind strongly to ECM proteins. A wound healing model with PlGF-2 $123-144$-fused PDGF-BB and VEGF-A demonstrated accelerated wound closure with increased granulation tissue when compared to wild-type PDGF-BB and VEGF-A. The study underscored that the affinity binding of VEGF to ECM is important to secure growth factor activity and to modulate growth factor signaling for angiogenesis, encouraging more studies to follow in other areas that aim to boost growth factor functions through biomaterials.

Based on this principle, a recent study engineered an immune-modulating angiogenic biomaterial and applied it directly to the stroke cavity to promote tissue formation de novo [211]. The HA hydrogel was modified to degrade both by hyaluronidase and MMP. Of note, heparin nanoparticles $(\mathrm{nH})$ were introduced to load VEGF as $\mathrm{nH}$ has the ability to bind growth factors [212]. When the formulated HA gels $+\mathrm{nH}$ with various VEGF clustering densities were introduced into the stroke cavity, a highly clustered VEGF hydrogel could induce the formation of a robust, mature and highly developed vascular bed as well as patterned axonal ingrowth along the vessels (Fig. 22). Based on the results, the clustered nanoparticle presentation of VEGF in the hydrogel was proposed to induce the differential VEGF receptor 2 phosphorylation and downstream p38 signaling which could ultimately promote the elements of vascular network development.

As demonstrated in the above findings, the matrix binding of angiogenic factors through heparin is considered a promising approach. Ishihara et al. [213] have recently found the naturally occurring laminin heparin binding domain (HBD) have high affinity to various growth factors (Fig. 23). In fact, laminin binds to growth factors through HBD located in the $\alpha$ chain laminin-type G domains which also bind to syndecan cell-surface receptors. These receptors are responsible for the attachment of fibroblasts and endothelial cells. The authors designed the peptide form of lamin HBD (LAMA3 $3043-3067$ ) and introduced to fibrin matrices. In a diabetic type II wound healing model, the engineered hydrogels showed accelerated wound healing with improved angiogenesis owing to improved growth factor delivery capacity, highlighting the effectiveness of angiogenic growth factor delivery through the matrix-affinity binding approach.

\subsection{Presentation of integrins and synergism with growth factors}

While the stimulation of growth factor receptors is a key strategy to induce angiogenesis, integrins have recently shown to be critically involved in a series of EC functions, including polarization, migration, and tubular formation [214-215]. Many different integrin subsets were found to get involved in various behaviors of ECs. For example, $\beta_{1}$ subset is known to control mural cell adhesion and spreading, providing stability to the wall of blood vessels (Fig. 24) [216]. Furthermore, biomaterials were often engineered with different sets of integrins (i.e., $\alpha_{5} \beta_{1}, \alpha_{2} \beta_{1}, \alpha_{v} \beta_{3}$ ) to activate integrin-mediated EC functions [61]. For example, selfassembled monolayers of various functional groups $\left(\mathrm{CH}_{3}, \mathrm{NH}_{2}, \mathrm{COOH}, \mathrm{OH}\right)$ were produced on Au-coated glass and the EC adhesion and migration were examined [217]. The surface with $\mathrm{COOH}$ group revealed high affinities for $\alpha_{2}, \alpha_{5}, \alpha_{\mathrm{v}}$ and $\beta_{3}$ subunits, significantly regulating the EC adhesion and migration. Furthermore, the Rho GTPases and (p) focal adhesion kinase expression was upregulated in the order; $\mathrm{CH}_{3}>\mathrm{NH}_{2}>\mathrm{OH}>\mathrm{COOH}$, implying the surface chemistry is important for presenting different integrin subsets and the corresponding endothelial integrin-mediated signalings. Another study designed SVVYGLR peptide-coated micropatterns $(10,50$ and $100 \mu \mathrm{m})$ on polyethylene terephthalate membrane. The peptide patterns were shown to modulate EC interactions via integrin receptor, and particularly narrow micropatterns (10 and $50 \mu \mathrm{m})$ had increased cell migration and tubular formation [218].

A recent study by Li et al. [219] has highlighted the different roles between the integrin subsets played in vascularization processes and the mechanisms underlying the event. The authors compared surfaces modified with two heterodimers of integrin, viz., $\alpha_{v} \beta_{3^{-}}$and $\alpha_{3} / \alpha_{5} \beta_{1}$. Compared with $\alpha_{v} \beta_{3}$-specific surface, $\alpha_{3} / \alpha_{5} \beta_{1}$-specific surface promoted cell-cell contacts by increasing the amount of vascular endothelial-cadherin at cell-cell junctions. In a murine stroke model, a significant increase in vascular area was noted for $\alpha_{3} / \alpha_{5} \beta_{1}$-specific group, suggesting a strong role in activating angiogenesis, however, a decreased vascular permeability was observed with $\alpha_{3} / \alpha_{5} \beta_{1}$-integrin that needs to modulate the vascular patterning after stroke, thus underscoring the proper targeting of integrin subsets depending on target diseases and angiogenesis patterns (Fig. 25). The finding that the angiogenesis pattern is critically altered by the integrin binding $\left(\alpha_{\mathrm{v}} \beta_{3}\right.$ and $\left.\alpha_{3} / \alpha_{5} \beta_{1}\right)$ may be potentially useful in designing biomaterials to regulate vascular patterning and VEGF-induced vascular permeability.

Fig. 22. Heparin-bound VEGF for post-stroke angiogenesis and vascular remodeling. (A) This study aims at designing and testing injectable proangiogenic regeneratives for stroke through the precise 3D presentation of VEGF with different packing density, binding to heparin nanoparticles in hyaluronic acid (HA) hydrogel. (B\&C) Mice injected with HA gel that contained the hcV (heparin particles with high cluster VEGF) condition (HA gel $+\mathrm{hcV}$ ) significantly increased the endothelial cell area (glucose transporter 1 (Glut-1)), proliferation (BrdU) and pericyte (platelet-derived growth factor receptor- $\beta$ (PDGFR- $\beta$ )) area in and around the stroke cavity compared to HA gel that contained the lcV (heparin particles with low cluster VEGF), nH (Heparin nanoparticles), Vs (soluble VEGF) or HA gel only, shown in images and their quantification. Taken together, highly clustered VEGF bonded with heparin nanoparticles (hCV) integrated into cell-degradable HA hydrogel induced a mature and highly developed vascular bed within in the stroke cavity along with axonal ingrowth. Adapted from Nih et al., Nature Materials, 2018 [211]. 

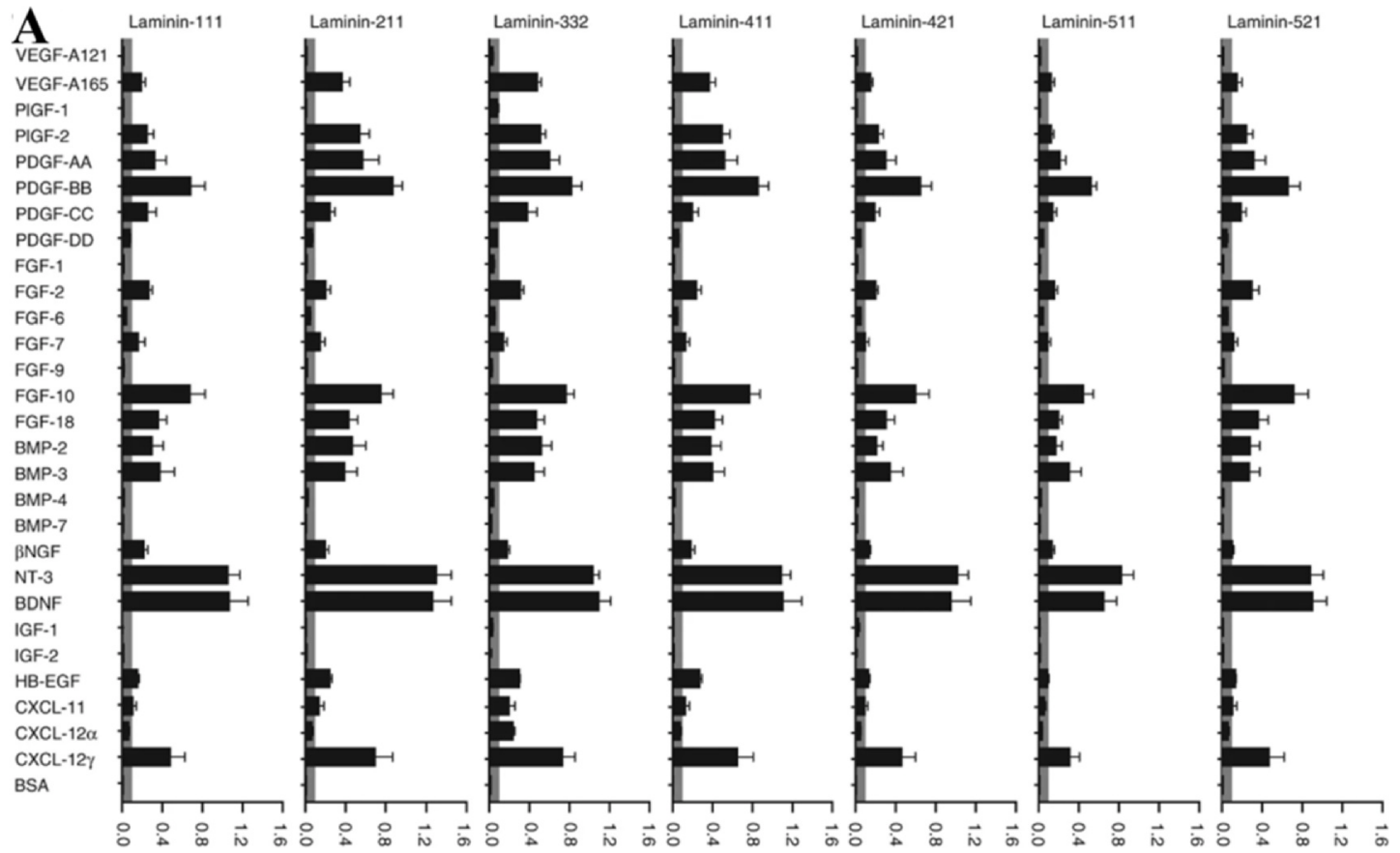

Fig. 23. Covalent incorporation of laminin heparin-binding domains (HBDs) into fibrin matrices improves retention of growth factors (GFs) and wound healing in vivo. (A) Laminin isoforms $(-111,-211,-332,-411,-421,-511$, and -521$)$ promiscuously bind to myriad growth factors (GFs) and chemokine with high affinity, measured by ELISA $(450 \mathrm{~nm}$ ). (B) Excess heparin inhibits GF-laminin binding, supporting that laminin heparinbinding domains (HBDs) mediated the interactions with GFs. (C) Location of HBDs was revealed in the $\alpha$ chain laminin-type G (LG) domains. (D) GF in vitro and in vivo retention in fibrin matrices is enhanced by incorporating laminin HBD peptide. (E) Delivering GFs and laminin HBD peptide enhances skin wound healing. Adapted from Ishihara et al., Nature Communications, 2018 [213].

The fact that the activation of integrins enhances angiogenesis motivates one important strategy: co-signaling of integrin and growth factor receptor. For example, the mediation of integrin $\alpha_{v} \beta_{3}$ and VEGFR-2 through c-SRC tyrosine kinase was shown to activate EC migration and tubulogenesis (Fig. 26A\&B) [220]. Garcia and coworkers [56,221] utilized the integrin/VEGF signalings through design of hydrogels. They engineered VEGF-functionalized PEG with different peptides; either $\alpha_{2} \beta_{1}$ integrin-specific peptide GGYGGGP(GPP) 5GFOGER(GPP)5GPC(GFOGER) or $\alpha_{v} \beta_{3}$ integrin-targeting peptide GRGDSPC (RGD). When tested in a critical-sized bone defect, the VEGF-GFOGER hydrogel showed higher bone formation with increased vascularization than the VEGF-RGD hydrogel [221], signifying that not only the integrin targeting is beneficial for vascularization in bone but also the type of integrin that cosignals with angiogenic factor VEGF is important in such process. Here, the $\alpha_{2} \beta_{1}$ integrin seems to be more potent than the $\alpha_{\mathrm{v}} \beta_{3}$ integrin in co-signaling with VEGF for the vascularization in bone. The co-activation of integrin with VEGF receptor can also be possible through the mediating action of FN. One recent study demonstrated that FN molecule assembled onto PEA surface could present specific domains that are beneficial for bindings of both integrin ( FNIII $_{9-10}$ ) and growth factor (FNIII $12-14$ ) (Fig. 26C) $[56,222]$. The fibrillar organization of FN was highly effective in promoting synergistic integrin/VEGF signaling and the resultant vascularization. Interestingly, the FN prepared onto PMA surface did not allow the integrin/VEGF co-presentation and the synergistic vascularization function. This study highlighted again the potential of integrin/VEGF co-signaling in vascularization, and the importance of biomaterials surface that can be designed strategically for the co-signaling.

\section{Scaffolding for cellular constructs}

\subsection{Cellular crosstalks in $2 D$ co-cultures}

For tissue regeneration, different types of cells work cooperatively to form vascularized network and produce matured tissue matrix. Below are described the cellular crosstalks in the angiogenic events of diverse tissues.

\subsubsection{Bone angiogenesis}

For the case of bone repair, the VEGF/BMP-2 paracrine signaling take a major part in stimulating both angiogenesis and osteogenesis. Generally, it is known that VEGF regulates the differentiation of stem cells to ECs while BMP-2 to osteoblasts, both cytokines promote the homing of MSCs and initiate the migration of ECs and osteoblasts from the surrounding tissues. The homing and migration of cells, and particularly the arrest of MSCs to relevant sites by transmigration across the endothelium is clinically relevant for the regeneration process as the MSCs recruited have high potential to differentiate into appropriate lineage (Fig. 27) 
B
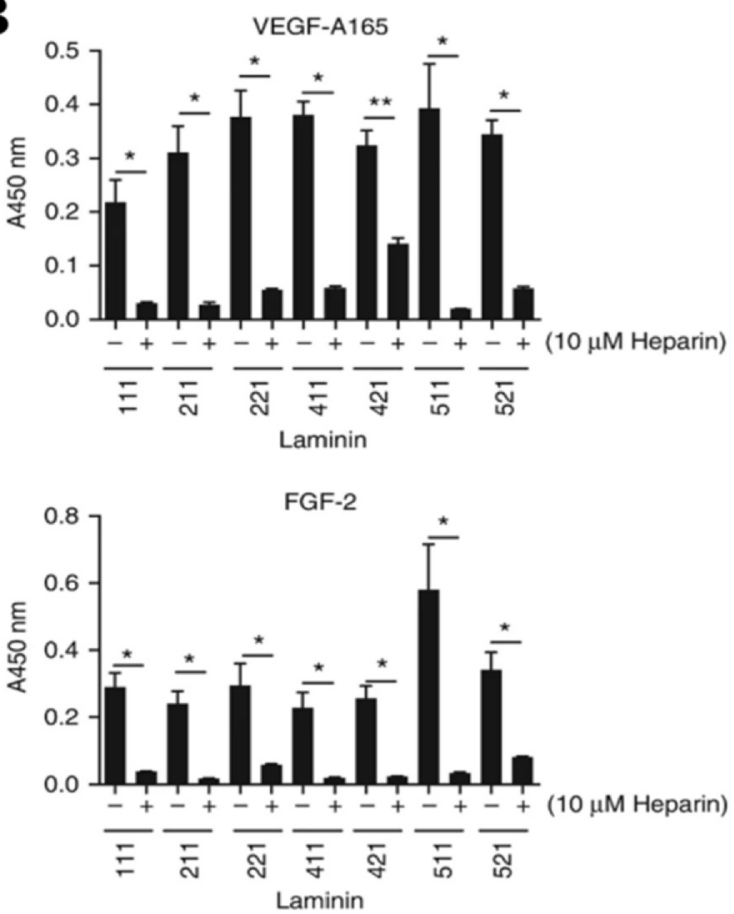

D
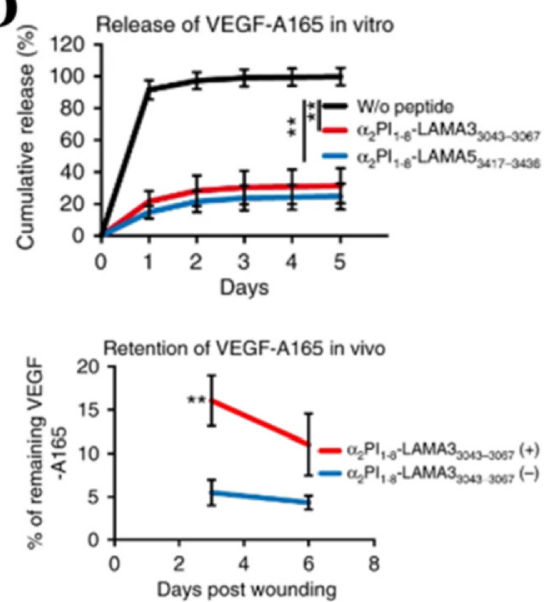

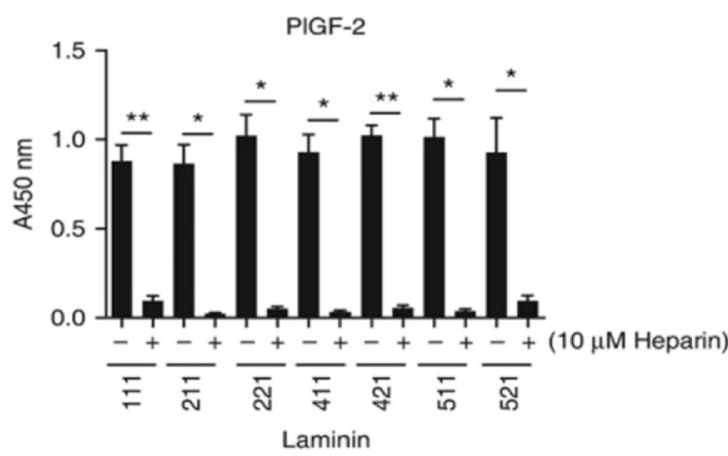

C
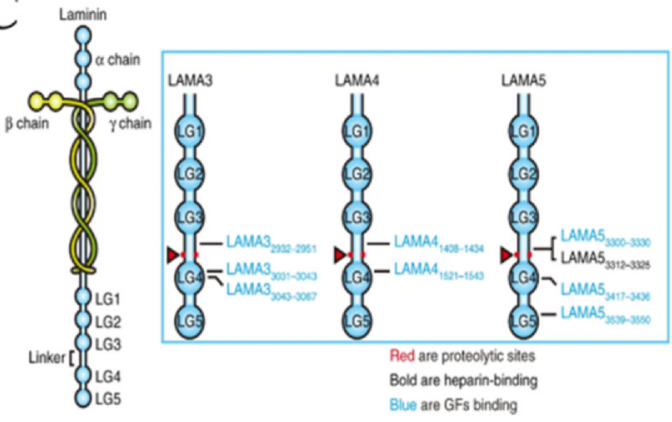
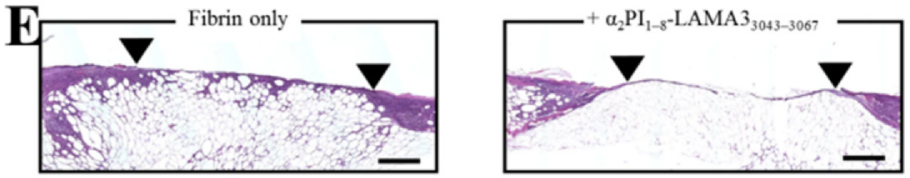

+ VEGF-A165 and PDGF-BB

$+\alpha_{2} \mathrm{PI}_{1-8}$-LAMA $3_{3043-3067}$
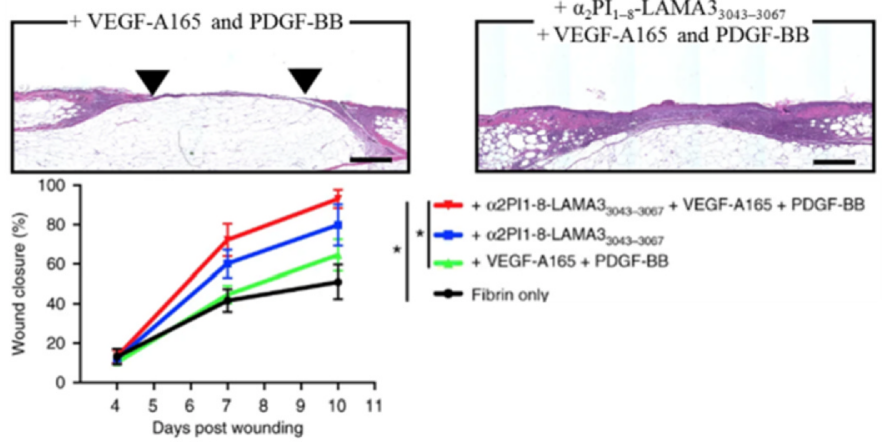

Fig. 23. (continued)

\section{[223-224].}

VEGF not only initiates angiogenesis but also has the ability to promote osteogenesis. This unique potential of VEGF, particularly its isoform VEGF-A on osteoblasts has been evidenced in the increased ALP and bone nodule formation [225]. Likewise, BMPs can contribute to the development of vascular system. BMPs are growth factors that fall into TGF- $\beta$ family [226]. Among the subgroups, BMP2/4/6/9/10 are importantly recognized by the endothelium [227]. To facilitate BMP signaling, ECs have specific cell surface receptors (activin receptor-like kinase (ALK)1, ALK2, ALK3, and ALK6) to which dimeric ligands of BMP attach. When BMP binds to the receptor, it initiates phosphorylation of SMAD1/5/8 proteins. This phosphorylated SMAD form complexes with SMAD4 which in turn binds DNA and other transcription factors leading to SMAD pathway. The BMP signaling that controls various functions in ECs is modulated by endoglin and BMP-binding EC precursor-derived regulator. It is thus clear that VEGF and BMP cross-stimulate osteogenic and angiogenic pathways, respectively. Importantly, the BMP/VEGF signaling is activated under hypoxia that is considered a major angiogenic trigger. Under hypoxia, BMPs are secreted from the vessels and combine with other pro-angiogenic cytokines, resulting in the activation of tip cells in vessels [228].

To examine and optimize the paracrine and direct signalings of cellular crosstalks, many studies have co-cultured different types of cells that take part in angiogenesis (ECs, EPCs, MSCs) and osteogenesis (osteoblasts, osteoblast progenitor cells, MSCs) [229]. Current findings in the 2D co-cultures of cells can help designing 3D cultures with biomaterial scaffolds for vascularization and bone 
A

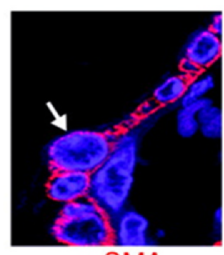

$\alpha-S M A$
Control

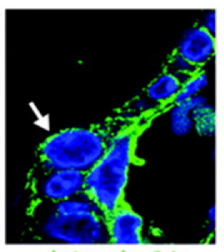

integrin $\beta 1$

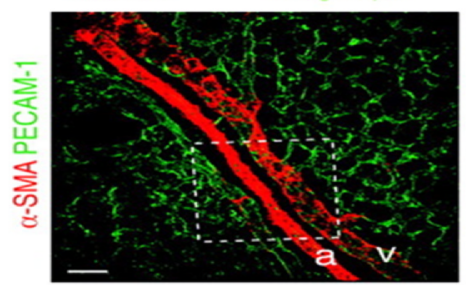

Inactivation of integrin $\beta 1$

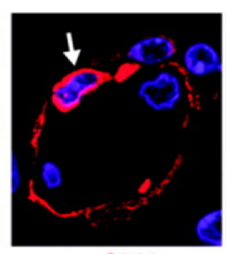

$\alpha$-SMA

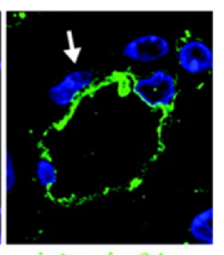

integrin $\beta 1$

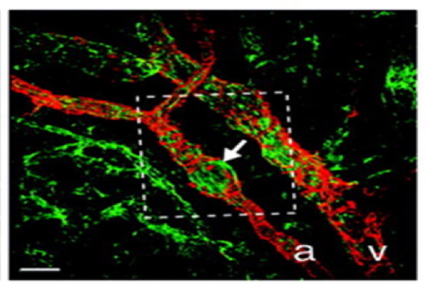

B Control
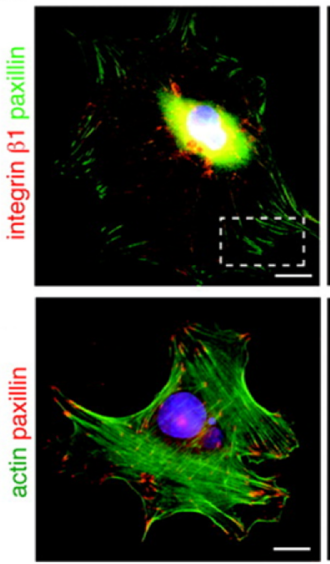

- integrin $\beta 1$
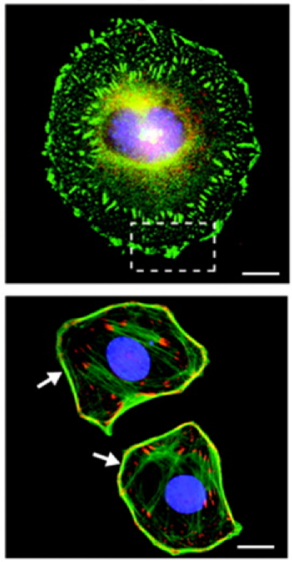

Fig. 24. Integrin $\beta 1$ regulates vascular smooth muscle cell adhesion, spreading, and blood vessel wall stability. (A) Inactivation of the integrin $\beta 1$ subunit (Itgb1) with a Cre-loxP approach in mice leads to the absence of integrin $\beta 1$ in vascular smooth muscle cells but not the endothelium. The presence of vascular aneurysms with local distensions of both arteries and veins was detected. (B) Absence of integrin $\beta 1$ expression induced short and disorganized focal adhesion in comparison with control cells, leading to cellular morphology (more round, poorly spread, and lack cellular protrusions). Adapted from Sabu et al., Circulation Research, 2008 [216].

formation. Among other co-cultures, ECs were often cultured with osteoblasts or MSCs for bone vascularization. For example, HUVECs or outgrowth endothelial cells (OECs) were co-cultured with osteoblastic cell line (MG63) [230]. Interestingly, the OECs with MG63 formed interconnected vascular networks whereas the HUVECs with MG63 did not, suggesting different capacity of ECs in signaling with osteoblasts and also presumably limited potential of osteoblastic cell line in communication with ECs, because many studies have shown the network formation of HUVECs with other cells including primary osteoblasts and MSCs [231-232]. Apart from ECs, other types of cells were also used to stimulate osteogenesis during co-cultures. For example, de-differentiated fat cells were co-cultured with periodontal ligament stem cells to enhance the osteogenic differentiation for periodontal regeneration [233]. When co-cultured indirectly through a transwell system, the RUNX2 expression was significantly upregulated with concurrent inhibition of PPAR $\gamma 2$, which was considered to drive osteogenic differentiation.

Along with the soluble molecular signalings, contact-dependent direct communications are also essential [234-236]. In one exemplar study, the growth factors produced by the cross-talk between primary osteoblasts and OECs were analyzed [237]. Among the 80 angiogenic genes screened by a polymerase chain reaction array, over 60 genes were upregulated at 1- and 4-week of cocultures. In particular, the cellular communication through gap junction molecule connexins was focused in the study. The analysis revealed Cx43 as a prime connexin involved in gap junctional cellular communication between the two different cells. This co-culture study gave an informative clue that endothelial and osteoblastic cells also crosstalk directly through gap junction connexins. Furthermore, a couple of studies on a chick embryo mandible model evidenced that the epithelial-mesenchymal cell membrane interaction played a key role in initiation of mesenchyme condensation for osteoblast formation [238-239].

Recently, three different cell types (OECs, osteoblasts, and macrophages) were also co-cultured to investigate if the macrophages might influence the formation of endothelial networks [240]. Of note, macrophages were demonstrated to induce angiogenic activation of OECs with upregulations of VEGF and adhesive molecules such as E-selectin and ICAM-1, ultimately leading to an increase in vascular morphogenesis while the osteogenic differentiation was not significantly altered by the macrophages. Due to the complexity of the tri-culture system, more studies need to be done to verify cellular mechanisms involved in the angiogenesis and osteogenesis. A similar study was also performed with neutrophils by co-culturing of HUVECs/osteoblasts to enhance angiogenesis and osteogenesis [241]. In fact, neutrophils are reported to promote angiogenesis [242-243]. A gene array platform was used to monitor the angiogenic and osteogenic genes during the tri-culture. Neutrophils were demonstrated to increase not only angiogenic genes such as VEGF, ANG, ANGPT2, EGF and FGF-2 but also BMPs (BMP1 to BMP5) and ECM proteins (COL1A1, COL3A1, COL5A1, COL10A1, and COL14A1). Furthermore, the calcification at days 14 and 21 was enhanced in tri-culture with respect to double-culture or mono-culture. The engagement of immune-related cells in angiogenesis and osteogenesis is essential during bone regeneration, thus, the effects of the cellular crosstalks need to be clarified which warrants further important area of investigation.

\subsubsection{Cellular crosstalks in angiogenesis of other tissues}

Among other tissues, skeletal muscle damaged needs sufficient angiogenesis for the repair process [244-245]. In skeletal muscle, blood vessels (capillaries) surround each myofiber and, when the muscle is damaged, the capillaries recruit inflammatory or stem cells, including muscle-derived stem cells (MDSCs) mainly originated from blood vessel walls, to start regeneration process [246]. The process of muscle regeneration comprises several steps: necrosis of the injured muscle cell, activation and proliferation of (activated) stem cells, differentiation and maturation of stem cells and formation of new muscle fibers and their remodeling. Particularly $t$ the early stage of muscle regeneration, muscle cells damaged undergo necrosis and start to trigger a cascade of inflammatory signals secreted from cells, which is also governed by angiogenesis [247]. In many studies, the improvement of 

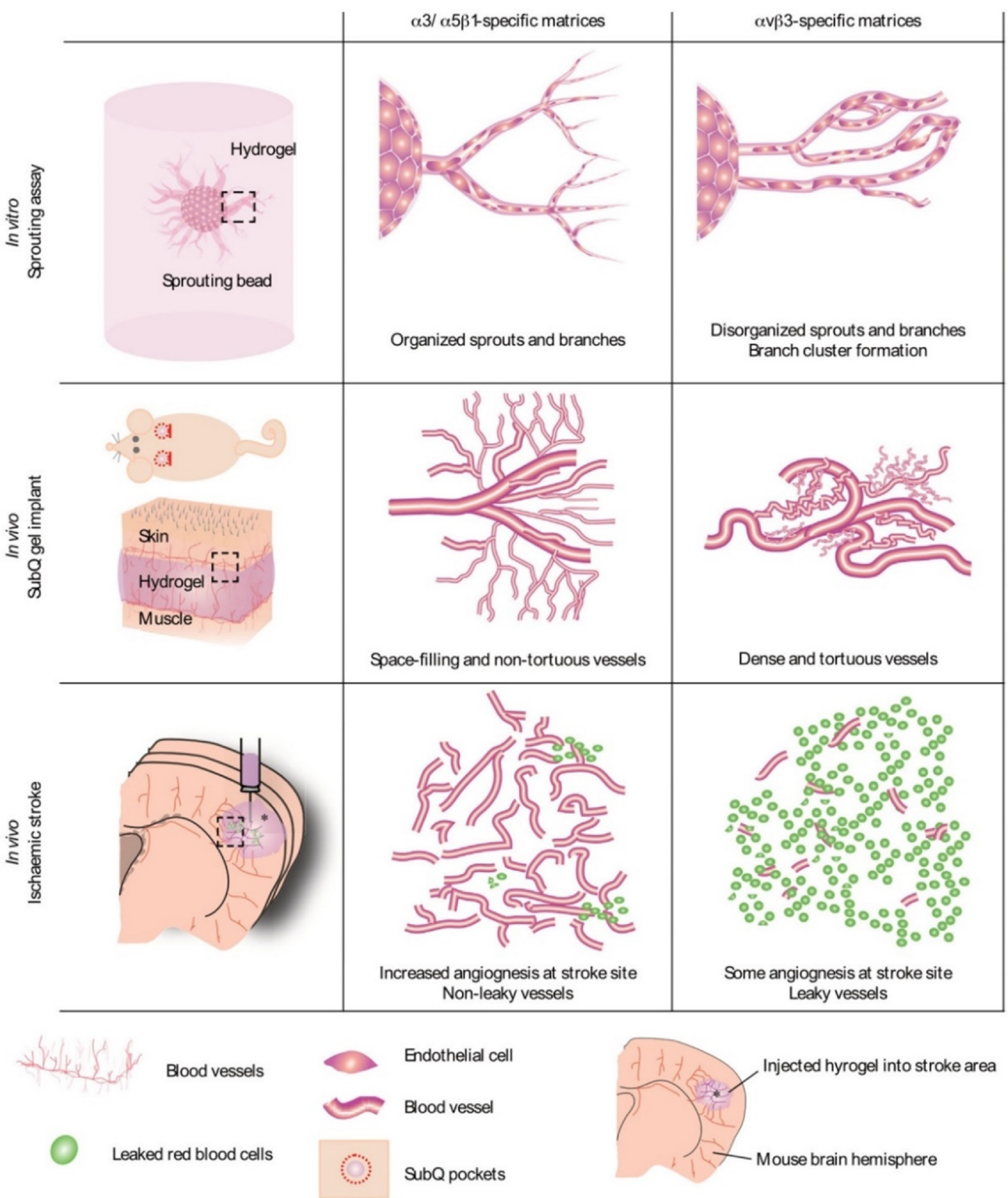

Fig. 25. Significance of integrin subsets $(\alpha 3 / \alpha 5 \beta 1$ and $\alpha v \beta 3)$ that exert different functions in angiogenesis. $\alpha 3 / \alpha 5 \beta 1$ specific hydrogel forms organized vascular sprouts and branches in vitro with space-filling and non-tortuous vessels in vivo. In an in vivo ischemic stroke model, this integrin specific hydrogel forms non-leaky vessels at stroke site. However, the $\alpha \mathrm{v} \beta 3$ specific hydrogel shows undesirable effects on the in vitro and in vivo studies. Adapted from Li et al., Nature Materials, 2017 [219].

angiogenesis during skeletal muscle regeneration has been highlighted as an optimal strategy, quickly recruiting muscle-regenerative stem cells and inflammatory regulating cells for healing process [248]. The angiogenic growth factor (e.g., VEGF) was thus used to accelerate the proliferation, migration and survival of MDSCs or myogenic cells (Fig. 28A\&B) [249-250]. When the VEGF-transduced MDSCs were implanted, the fibrosis (non-functional scar tissue) of muscle tissue could be significantly mitigated [251]. Also, skeletal muscle grafts were engineered by means of a direct revascularization of ECs on a resorbable scaffold, which was capable of releasing angiogenic VEGF and FGF2, exerting synergistic angiogenic effects (Fig. 28C-E) [252]. Here, an in vitro triculture system of three different types of cells (ECs, myoblasts, and foreskin fibroblasts) was used for revascularizing muscle graft. After three-week culturing, semi-organized network-like structures and muscle fibers were formed, which significantly enhanced grafting efficacy with accelerated perfusion and capillary density in vivo. The modulation of immune system via angiogenesis has recently been highlighted 


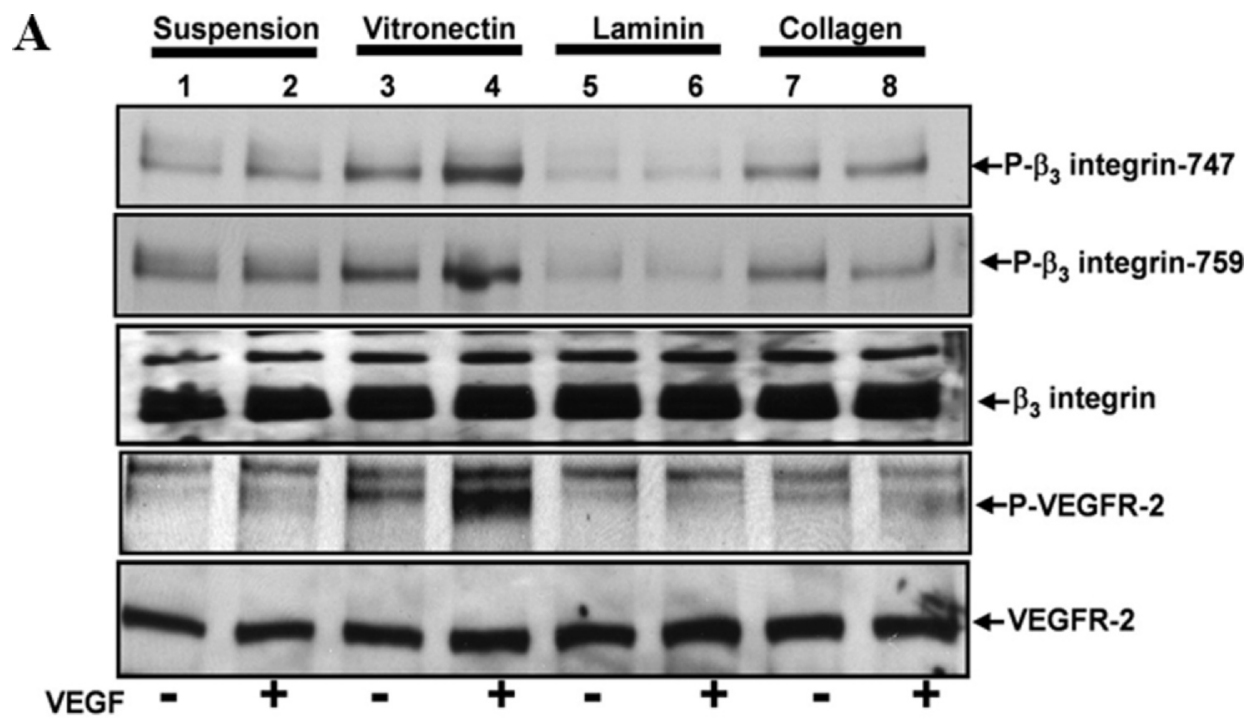

Fig. 26. Co-signaling of integrin and growth factor (receptor) for angiogenesis. (A) Cross-activation between $\beta 3$ integrin and tyrosine kinase receptor 2 for VEGF receptor (VEGFR-2) was revealed in ECs plated on the $\alpha \mathrm{v} \beta 3$ ligand vitronectin. Vitronectin, but not laminin or collagen, was capable of inducing $\beta 3$ and VEGFR-2 tyrosine phosphorylation, which was synergistically augmented on VEGF treatment. Adapted from Ganapati et al., Circulation Research, 2007 [220]. (B) Protease-degradable, VEGF-loaded poly(ethylene glycol) (PEG) hydrogels was functionalized with either a triple-helical, $\alpha 2 \beta 1$ integrin-specific peptide GGYGGGP(GPP)5GFOGER(GPP)5GPC (GFOGER) or an $\alpha$ v $\beta 3$ integrin-targeting peptide GRGDSPC (RGD). When applied to critical-size segmental defects in the murine radius, GFOGER-functionalized VEGF hydrogels exhibited significantly increased vascular volume and density with a larger number of thicker blood vessels compared to RGD-functionalized VEGF hydrogels, describing that the $\alpha_{2} \beta_{1}$ integrin seems to be more potent than the $\alpha v \beta 3$ integrin in co-signaling with VEGF for the vascularization in bone. Adapted from García et al., J Biomed Mater Res A, 2016 [221] (C) The co-activation of integrin with VEGF receptor can also be possible through the fibronectin. Adapted from Moulisová et al., Biomaterials, 2017 [56].

as an important strategy for muscle regeneration [253]. Immune cells recruited from blood vessels were demonstrated to play key regulatory roles in affecting muscle regeneration.

For neural tissues such as spinal cord and brain injuries, angiogenesis accelerates neuronal growth and functional repair. Neurogenesis and angiogenesis are closely linked through vascular production of stromal-derived factor 1 and Ang1, which promote post-injury neuroblast migration, neurogenesis and neuro-functional recovery [254]. Also, the angiogenic response following spinal cord or brain injury is regulated by the interplay with inflammatory signals [255]. Therefore, the prevailing inflammatory conditions following neural injuries can be mitigated by the use of angiogenic factors (i.e. VEGF and FGF2) with biomaterials [256-259]. Skin regeneration has also been studied in the context of angiogenesis, where the angiogenic stimulation crosstalks with surrounding inflammatory cells, fibroblasts, and epithelial cells, regulating the molecular networks in wound healing process [260]. The cellular crosstalk for skin wound healing and supportive roles of biomaterials are well described in a recent review [34].

\section{2. $3 D$ co-cultures with biomaterials}

Biomaterials provide unique substrate conditions to cells co-cultured whereby the various sets of cell-matrix interactions significantly influence the cellular crosstalk. Even with 2D substrate conditions, the surface properties (e.g., chemistry and nano/ microtopography) can modulate the intercellular signaling. For example, different ligand densities, driven by tailored surfaces with adhesive molecules or nanogrooves, can enhance cell-matrix interactions and the resultant paracrine signaling. For bioactive or degradable materials (e.g. calcium phosphates or silicate glasses for bone), the dissolved products involved in cell mitosis and angiogenesis (e.g., silicate, calcium) can stimulate the cellular crosstalks. A representative scenario of the ECs/MSCs crosstalk involves the expression of VEGF from MSCs, which activates VEGF receptor (KDR) in ECs and further expression of a set of angiogenic factors including eNOS as well as osteogenic regulator BMP-2, which in turn drives BMP-2 activated osteogenic differentiation of MSCs. In fact, biomaterials with a 3-dimensionality such as foamed scaffolds, fiber networks and hydrogels are commonly used to culture cells for tissue regeneration. Therefore, the effects of 3D biomaterials on the cellular crosstalks are much more intricate and potential. Below are discussed some representative studies carried out to co-culture cells with 3D biomaterials, with an emphasis on the role of biomaterials in cellular crosstalks and the resultant angiogenesis and tissues regeneration.

\subsubsection{D biomaterial co-cultures for bone angiogenesis}

Bioceramic foam scaffolds are the common type of biomaterials used to repair bone. Calcium phosphate ( $\beta$-TCP) scaffolds with pore sizes of 350-500 $\mu \mathrm{m}$ were used to provide a 3D matrix condition for HUVECs/MSCs (Fig. 29A-D) [261]. In this work, the cocultured group showed an earlier ALP activity than the mono-cultured MSCs group due to the secretion of BMP-2 by HUVECs. 
B

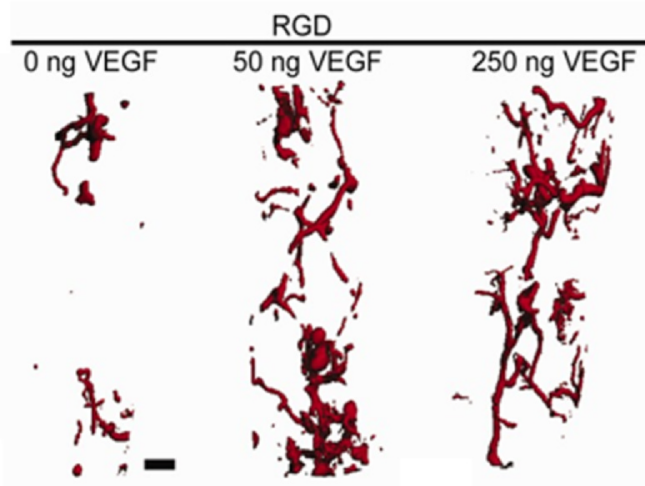

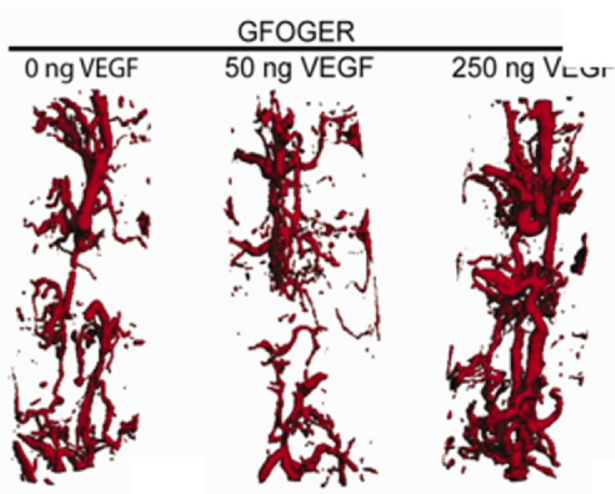
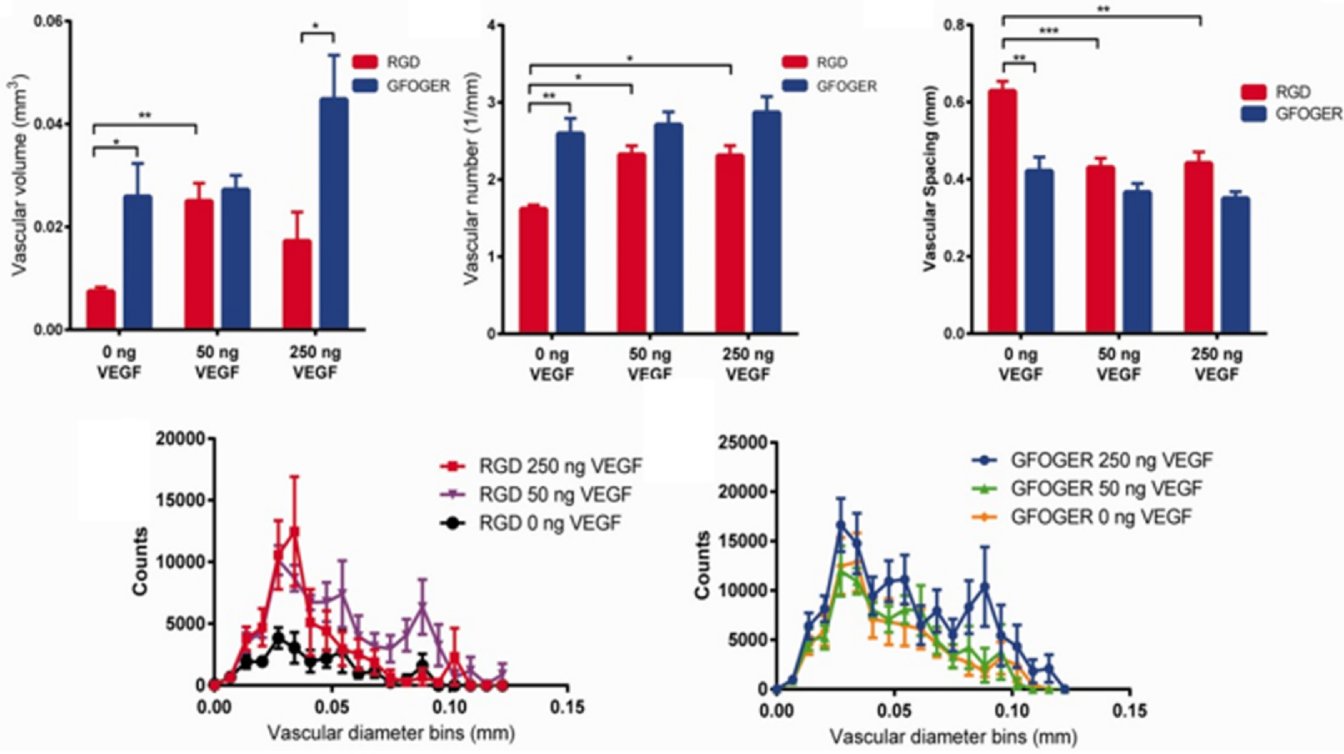

C
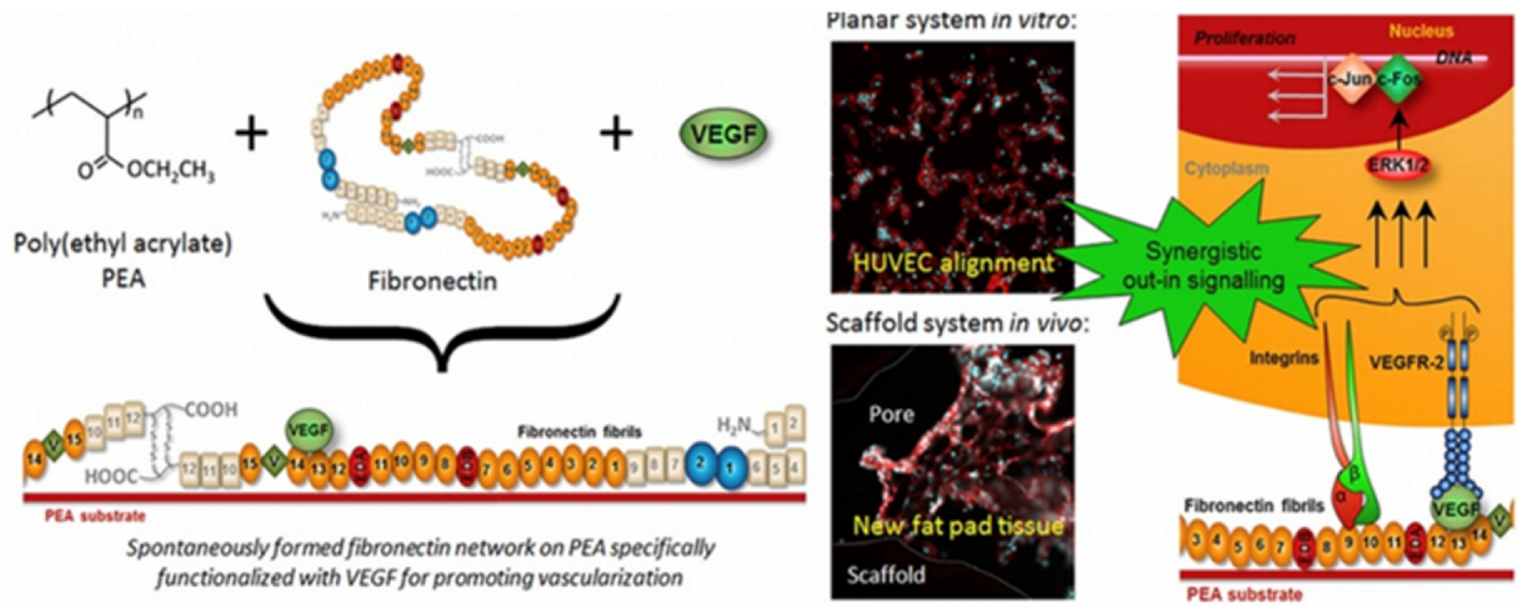

Fig. 26. (continued)

Interestingly, the expression of BMP-2 gene was higher in HUVECs than in MSCs, implying the released BMP-2 in the co-culture might trigger osteogenesis of MSCs. The role of BMP-2 produced by ECs in stimulating the osteogenic differentiation and mineralized vascular tissue formation was also noticed elsewhere [262]. Degradable polymeric scaffolds were also used to co-culture ECs/MSCs [263]. When seeded in PLGA scaffolds, the cells became closer together leading to more cell-cell contacts between heterogeneous 

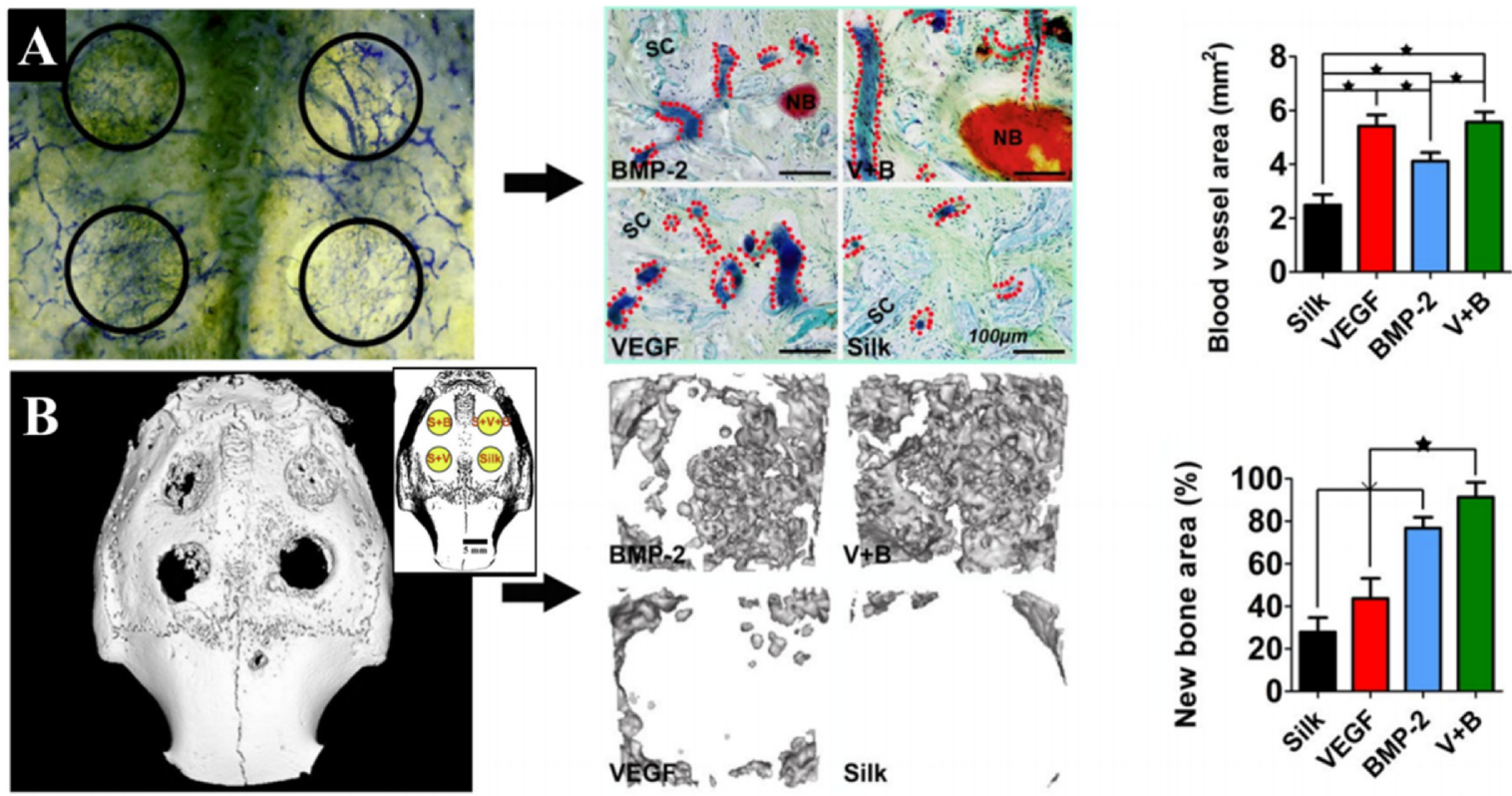

Fig. 27. Cellular crosstalks in bone angiogenesis through VEGF/BMP2 signaling. (A) Synergetic neo-angiogenesis in calvarias via VEGF/BMP2 coactivation (labelled with blue coloured Microfil) in the gross specimens and histologically stained sections (red dash lines indicating blood vessels, $\mathrm{SC}=$ scaffold, NB = new bone). Quantitative analysis of the local vessel area in the defects. (B) New bone formation at 12 weeks after implantation, captured by $\mu \mathrm{CT}$ and three-dimensional reconstruction image. The graph shows the percentage of new bone area in the different groups. ( $\star$, represents p < 0.05). Adapted from Zhang et al., Eur Cell Mater, 2014 [224].
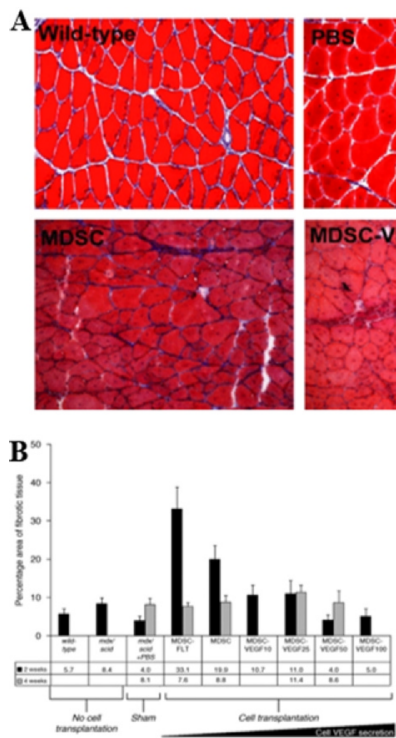
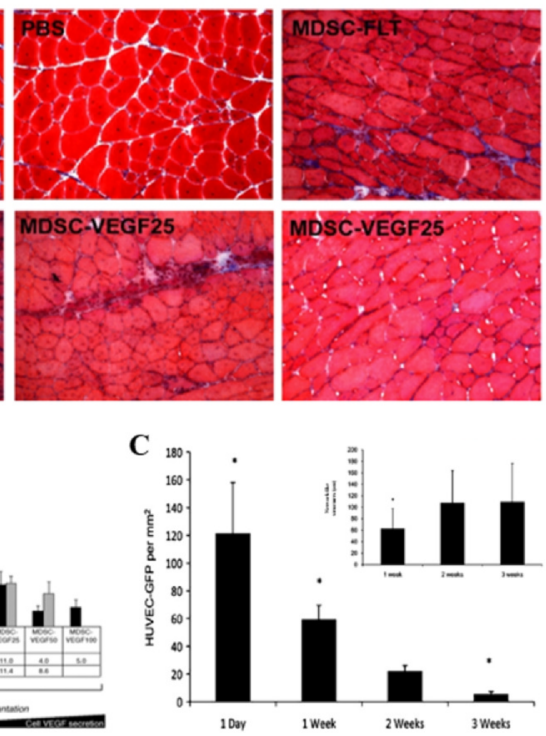
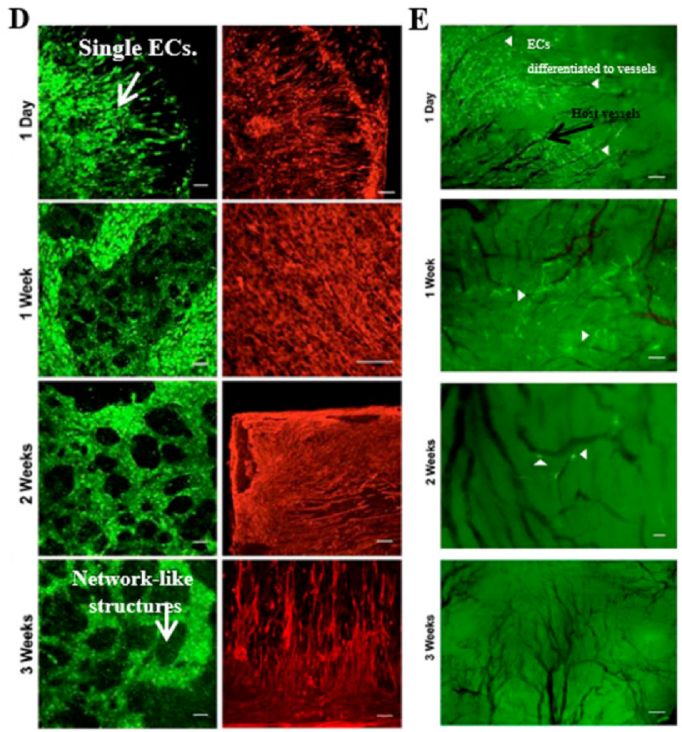

Fig. 28. Cellular crosstalks in angiogenesis for muscle regeneration. (A\&B) VEGF from ECs potentially modulates skeletal myoblast function. Transplantation of VEGF transduced muscle-derived stem cells led to an decrease in the amount of fibrosis as measured via trichrome staining, and as compared to noninjected tissue. Adapted from Antonia et al. [249]. (C-E) Engineered vessel consisting of ECs, myoblast and fibroblast on angiogenic bioscaffolds for functional skeletal muscle regeneration. Revascularized grafts were constructed using a triculture system of ECs, myoblasts, and fibroblasts and cultured in vitro for 3 weeks. (D) Over in vitro co-culturing time, single ECs gradually disappeared and anastomosed structures appeared dominantly (green: HUVEC-GFP \& red: desmin), quantified and shown in insert in (C). (E) When above engineered grafts were implanted in vivo, a full thickness segment of the abdominal wall of nude mice, ECs in grafts differentiated to vessels (white arrowheads) and integrated with host vessels (black) over in vivo integration time. Bar: $1 \mathrm{~d}-200 \mu \mathrm{m}, 1 \mathrm{wk}-100 \mu \mathrm{m}, 2 \mathrm{wk}-50 \mu \mathrm{m}, 3 \mathrm{wk}-200 \mu \mathrm{m}, \mathrm{n}=5$, p $<0.001$. Asterisks indicate statistical significance. Adapted from Jacob et al., Proceedings of National Academy of Science, 2012 [252]. 

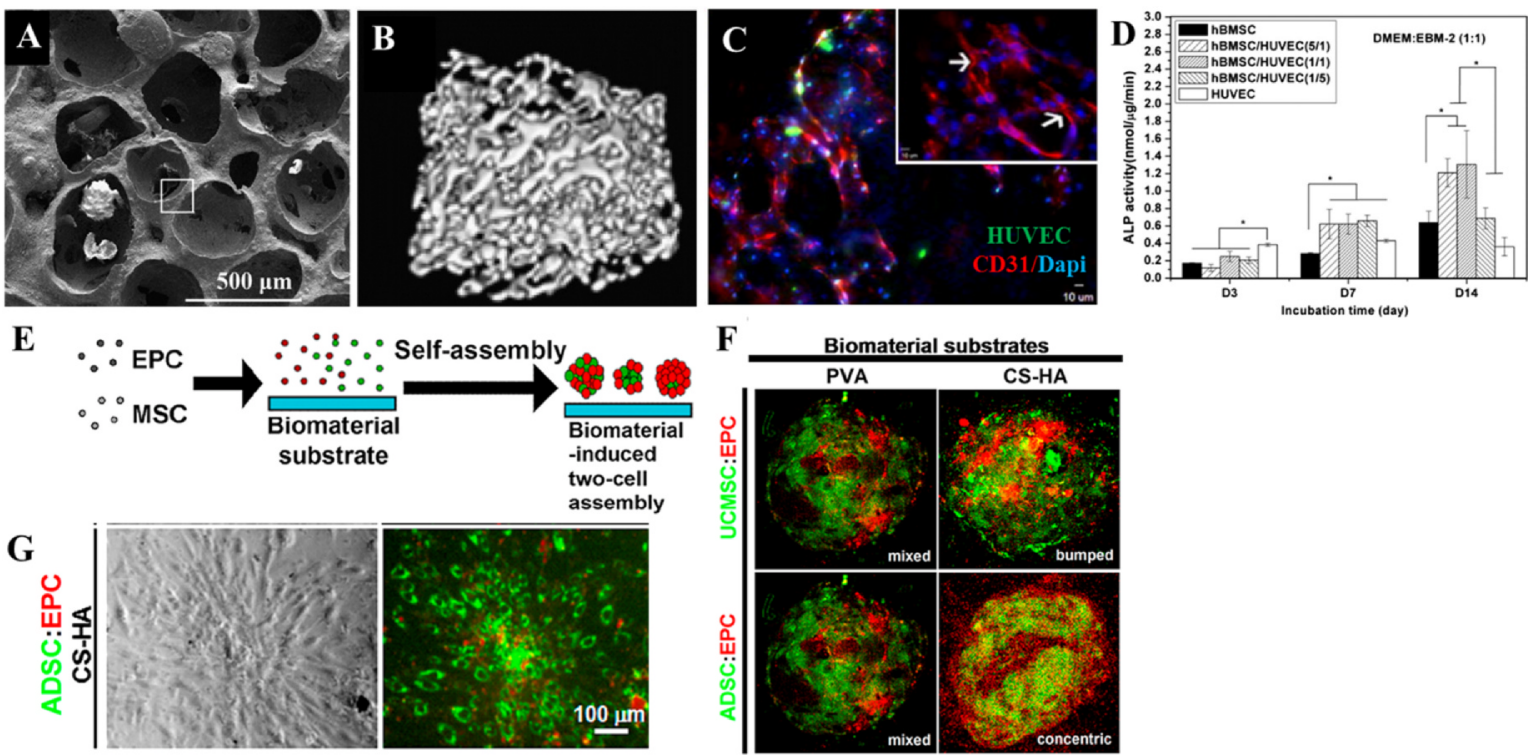

Fig. 29. 3D biomaterial co-cultures for bone angiogenesis. Bioceramic (biodegradable beta-tricalcium phosphate ( $\beta$-TCP)) was used to provide a 3D matrix condition for HUVECs/MSCs coculture. (A) SEM morphologies of the $\beta$-TCP scaffold and (B) 3D- $\mu$ CT images. (C) Confocal images of HUVECs/MSCs co-culture on $\beta$-TCP scaffolds at 14 days, indicating pronounced endothelial markers (CD31) at the cell-cell interface and formation of small sprouts (white arrow). (D) The increase of ALP activity was shown in co-culture compared to in monoculture on $\beta$-TCP scaffolds. An asterisk is used to indicate significant difference $(p<0.05)$. Adapted from Kang et al., Acta Biomater, 2013 [261]. (E) 3D spheroid self-assembled in MSCsEPCs coculture system on different substrate. (F) Representative confocal microscopy images depending on the substrate and cellfor the aforementioned three types of co-spheroids from umbilical cord (or adipose tissue) derived MSC-EPC 2:2, including the mixed spheroids, bumped spheroids, and concentric spheroids. (G) Among many conditions, the spreading of ADSC-EPC derived on CS-HA was the most rapid, indicating angiogenic potential. Adapted from Hsu et al., Biomaterials, 2014 [274].

cells than when cultured on 2D culture plate, which resulted in enhanced MSCs osteogenic differentiation. The rapid cellular aggregation in the 3D scaffolding matrix that allows more cell-cell interactions boosts crosstalk between ECs and MSCs which is required for vascularized bone formation in vivo. A similar effect of improved vasculogenesis by the closer cellular contacts was also observed when starch/polycaprolactone fiber meshes seeded with human osteoblasts and ECs were implanted subcutaneously $[15,264]$.

Among other forms of biomaterials, hydrogels provide cells with 3D microenvironments that mimic the native tissue [265]. As such, many earlier studies used hydrogels to interpret the interactions of ECs with osteoblasts or MSCs. For example, collagen-based gels were cultured with ECs/osteoblasts to investigate the sprouting and tubular formation [266]. Also, angiogenic signaling molecules (VEGF-165 and bFGF) were introduced in the 3D collagen gels to enhance the sprouting of ECs [267]. In the system, the autocrine/paracrine interactions of ECs/osteoblasts were shown to have angiogenic-stimulated osteogenesis [268-270], suggesting the importance of signaling molecules in the crosstalks within hydrogels. Matrigel, as a natural ECM hydrogel, was often used to coculture cells for vascularization and osteogenesis. The co-cultured EPCs and MSCs were shown to get much closer cell-cell contacts within the gels which could modulate angiogenic responses and expression of cytokines that are favorable for tubular formation of EPCs and osteogenesis of MSCs [271].

When co-cultured in hydrogels, the production of pro-angiogenic cytokines (VEGF, IGF-1, PDGF-BB and Ang-1) by the MSCs has also been highlighted in the crosstalks [272]. Alginate is a well-known hydrogel used for culture of cells. Since it lacks cell adhesive motifs, adhesion proteins or ligands are often introduced. Human osteoprogenitor cells (HOPs) and HUVECs were co-encapsulated in RGD-grafted alginate microcapsules which were then implanted in bone defects [273]. When cultured together with HUVECs in the microcapsules, the HOPs enhanced the VEGF secretion which in turn could induce HUVECs to secrete osteogenic molecules (e.g., BMP-2), thus promoting osteogenesis of HOPs. It is worth noting that the secreted molecules take longer time to diffuse out of the hydrogel than when they are in 2D culture that enables more effective cellular crosstalks. In addition, substrate, types of MSCs and ratios between MSC and ECs were optimized for making more angiogenic co-spheroid. When the two populations are seeded in 1:1, they are self-assembled in co-spheroids with different morphologies including randomly mixed, bumped, or concentric spheroids, which are influenced by cell-substrate or cell-cell interaction. In particular, the self-assembled concentric spheroids from adiposederived MSCs and EPCs showed a greater angiogenic effect in vitro (Fig. 29E-G) [274].

In another study, OECs and MSCs were co-cultured in silk fibroin hydrogel modified with IKVAV peptide (a sequence derived from laminin) [275]. While the mono-culture of OECs in silk fibroin hydrogel failed to form vascular networks, the co-culture formed abundant vascular networks, and the results were deemed to the production of pro-angiogenic cytokines from MSCs as well as ECM components like type I collagen that can mediate angiogenesis of OECs. The injectable hydrogel-based co-culture strategy was further proposed to be a promising treatment option for acute vascular injuries. 
To present the signaling molecules more effectively hydrogel networks were often functionalized. An in situ forming starPEGheparin hydrogels were synthesized where heparin has numerous binding sites for growth factors and adhesion ligands [276], and the ECs and different mural cells (MSCs, smooth muscle cells, dermal fibroblasts, and $10 \mathrm{~T} 1 / 2$ ) were co-cultured within the hydrogels. The co-culture of ECs with MSCs produced tubular networks with the highest density and longest branch length as evidenced similarly in other studies. Intriguingly, when the culture period was prolonged for one month, the ECs co-cultured with human dermal fibroblast or $10 \mathrm{~T} 1 / 2$ could produce dense tissue-like structures whereas those with SMCs or MSCs exhibited little change. It was speculated that various growth factors released through different crosstalks and their involvement in intercellular signalings might be responsible for the observed behaviors. Although more studies are warranted to underlie the events, the study highlights the important role of a hydrogel matrix that can sequester the soluble factors secreted from cells and then provide for their signalings for a long-term culture in 3D environments.

As demonstrated, the biomaterials used for the co-culture of cells provide physicochemical properties and 3-dimensionality effective for the cellular crosstalks in angiogenesis during tissue regeneration. Along with this, dynamic cultures were often used to allow cells to communicate under in vivo mimic conditions. Among the parameters in dynamic environments, fluid flow, hypoxia, $\mathrm{pH}$, and mechanical stress were widely studied. For example, under hypoxic conditions, 3D porous PCL scaffolds co-seeded with osteoblasts and HUVECs showed enhanced osteogenesis in a rotary cell culture system [277]. Also, studies have shown significantly increased aggregation of ECs and tubular formation as well as osteogenesis and calcification when cells were co-cultured in a perfusion bioreactor [278]. The cellular crosstalks and shear mechanical stimulation might have synergistic effects on both angiogenesis and osteogenesis.

One thing to note that the biomaterials-enabled 3D in vitro co-cultures do not normally lead to capillary formation as sufficiently as the in vivo conditions [279]. This implies that the 3D cellular responses in biomaterials might be more explicit in native tissue environments and the in vitro cultures might often not be relevant to model and predict the biomaterials role in 3D multi-cellular

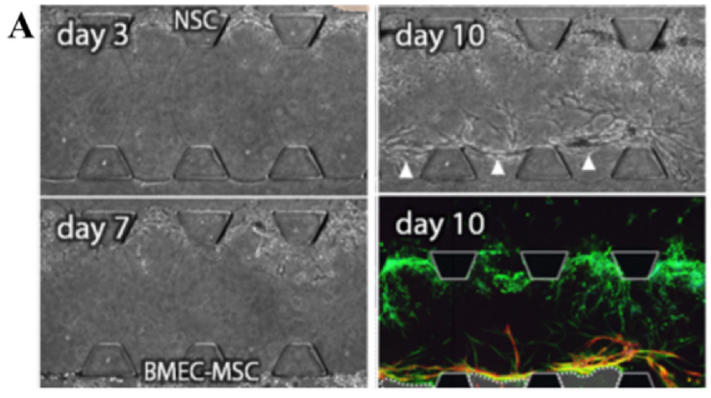

C In microfluidic device

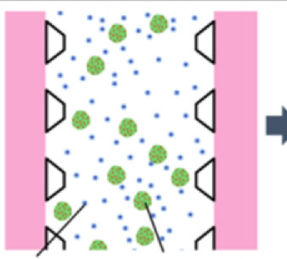

Human iPS-EC MN spheroid

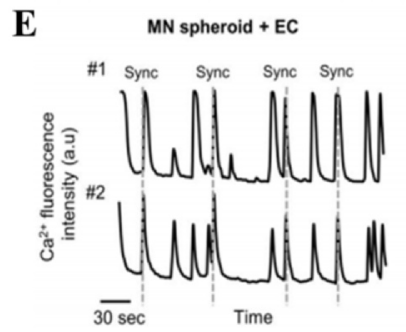

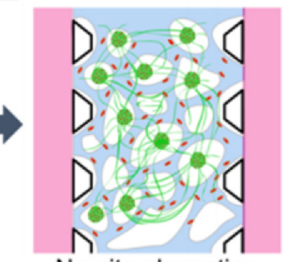

Neurite elongation

Vasculnaenesis

MN spheroid

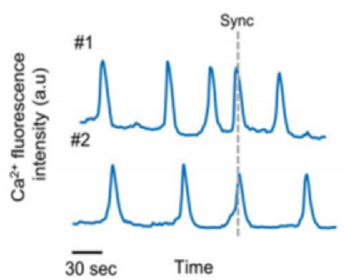

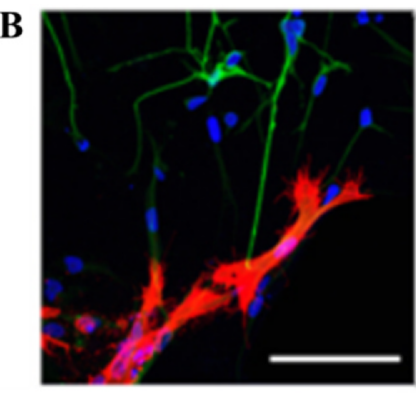

D

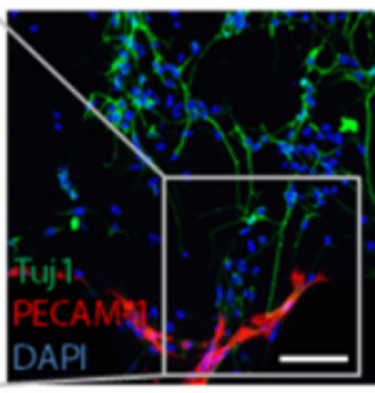

Tubulin/CD31

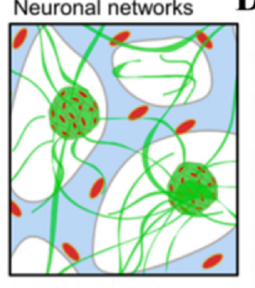

Vascular networks

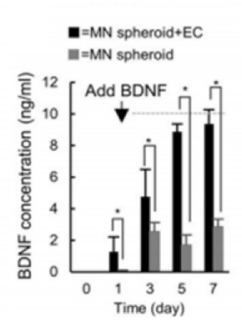

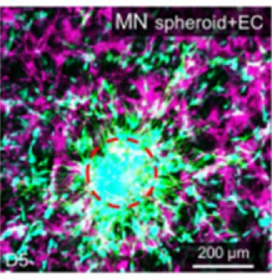
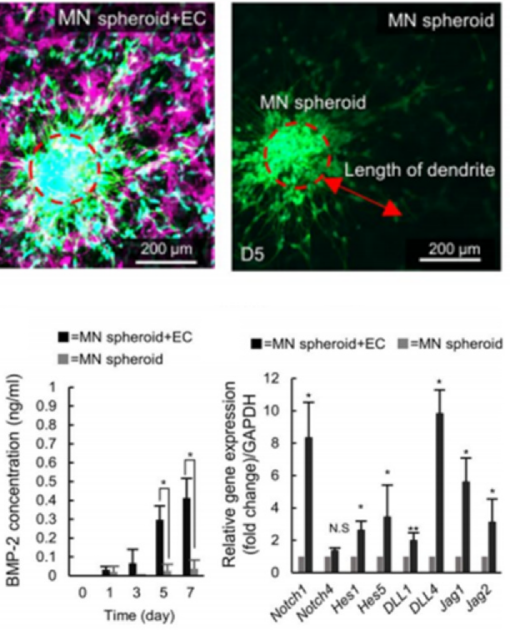

Fig. 30. 3D biomaterial co-cultures for neurovascular regeneration. (A-B) 3D fibrin-Matrigel was optimized to construct neurovascular unit (ECsneural lineage cell). ECs (PECAM-1, red)-MSCs were seeded in fibrin-matrigel at 7 days later after neural stem cells (Tuj1, green) were pre-seeded in an optimized 3D ECM hydrogel condition to make neurovascular tissues on day 10, (B) which preserved the typical neurovascular unit consisting of interaction between capillary-like structures and neuritis. Scale bars, $100 \mu \mathrm{m}$. Adapted from Hiroyuki et al., Sci Rep, 2017 [282]. (C-E) Further, 3D networks were established by co-culturing human embryonic stem (ES)-derived motor neuron spheroids and ECs in microfluidic devices, revealing longer neurite elongation, significantly upregulated neuronal connectivity as measured by $\mathrm{Ca}^{2+}$ oscillation, and growth factors secretion from coculture (motor neuron spheroid + ECs) condition compared to motor neuron spheroid alone. $\mathrm{n}=3$; ${ }^{*} \mathrm{P}<0.01$, two-way ANOVA. All error bars \pm SD. Adapted from Tatsuya et al., Sci Rep, 2018 [283]. (For interpretation of the references to color in this figure legend, the reader is referred to the web version of this article.) 
performance [280].

\subsubsection{D biomaterial co-cultures for nerve and muscle angiogenesis}

The 3D co-culture studies for angiogenesis have also been carried out for nerve and muscle tissues (Fig. 30). Many 3D microfluidic culture models aim to mimic the neurovascular unit in brain, including neurons, glial cells, blood vessels and extracellular matrix. An earlier study has optimized the hydrogel type for preserving EC biological functions in a microfluidic platform. Among the tested groups of type I collagen, fibrin, and their combination at different concentrations, the fibrin gel ( $2.5 \mathrm{mg} / \mathrm{ml}) \mathrm{was}$ shown to generate highly matured microvascular networks [281]. Furthermore, the fibrin-Matrigel was selected for the neurovascular unit, where ECsMSCs were seeded in the gel at 7 days after the induction of neural stem cells to neuronal growth. The hydrogel based 3D co-culture system could preserved the typical neurovascular unit that recapitulates the interaction between capillary-like structures and neurites [282]. Recently, 3D networks were also established by co-culturing human ES-derived motor neuron spheroids and ECs in a microfluidic device [283]. The 3D co-culture system (motor neuron spheroid + ECs) revealed highly elongated neurites, upregulated neuronal connectivity, and growth factors secretion when compared to the culture of motor neuron spheroids only, demonstrating the importance of endothelial-neural crosstalks in the stimulation of functional neuronal activity.

For muscle regeneration, culturing muscle cells with ECs through biomaterials has been shown to generate functional skeletal muscle grafts with enhanced vascularization and innervation, similar to native muscle. In a simple approach, myoblasts cultured in a 3D fibrin gel under dynamic tension became aligned myofibers that could generate forces (Fig. 31) [284]. Furthermore, the myoblasts were seeded with ECs for prevascularization. Interestingly, these 3D constructs differently formed aligned vascular networks depending on the ratio of mixture cells, and an optimal combination (50-70\% are muscle cells and 30-50\% are ECs) in a fibrin matrix was proposed [285]. Another approach to prevascularize engineered muscle graft is seeding ECs and fibroblasts with myoblasts onto a fibrin-PLG 3D scaffold, leading to prominent in vivo muscular functionality compared to cell-free or single cell counterpart [286]. The aligned tissue engineered myofibers made with 3D gel or hard scaffolds also revealed acetylcholine receptor clustering, with enhanced in vivo nerve innervation and angiogenic potential [287]. In a recent approach of biomaterial-enabled 3D muscle regeneration, the 3D printed vascular grafts were fabricated with bioinks (i.e. alginate-collagen) containing ECs, smooth muscle cells, and human amniotic fluid-derived stem cells, which subsequently connected to muscle grafts [288].

A
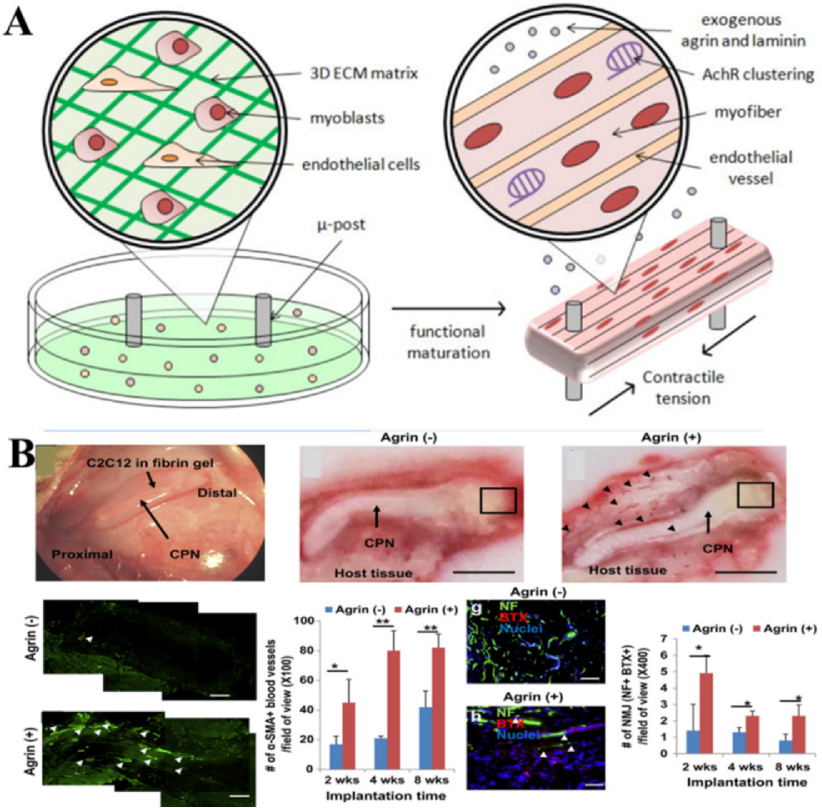
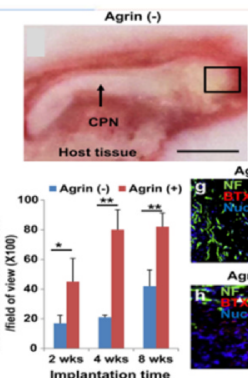
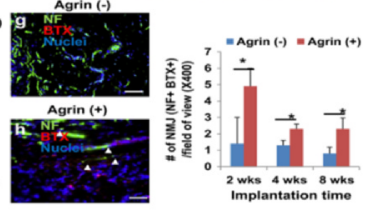

Agrin (+)

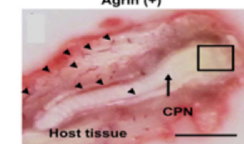

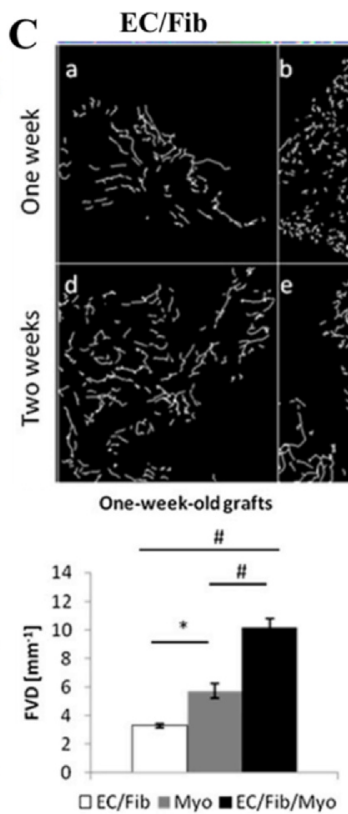

Myo

EC/Fib/Myo

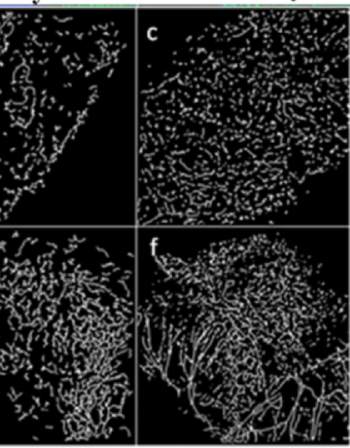

$\square E C / F i b=M y o=E C / F i b / M y o$

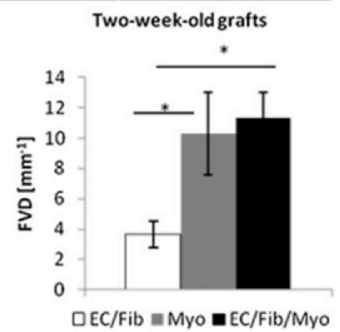

Fig. 31. 3D biomaterial co-cultures for muscle regeneration by angiogenic stimulation. (A-B) Suggested strategy for vascularizing muscle cells combined with biomaterials for regeneration. (A) Engineered skeletal muscle cells and ECs seeded into a fibrin gel were cultured in dynamic tension force to form aligned myofibers, which may also include exogenous agrin and laminin to promote acetylcholine receptor clustering for nerve innervation. (B) Numerous large blood vessels and neuromuscular junction (NMJ) were observed in the grafts treated with agrin, whereas there was no remarkable blood vessels or NMJ in the implant without agrin treatment. Student $t$-test, $* P<0.05, n=3$ from three individual animals, $n=3-5$ animals). Scale bars: $1 \mathrm{~mm}$ (b and c), $500 \mu \mathrm{m}$ (d and e), and $50 \mu \mathrm{m}$ (g and h). Data represent mean \pm SD. Adapted from Brain et al., Curr Opin Biotech, 2017 [289]. (C) Another approach to prevascularize engineered muscle graft is seeding ECs and fibroblasts with myoblasts onto a fibrin-PLG 3D scaffold, giving prominent in vivo muscular functionality compared to cell-free or single cell counterpart. *P $<0.05$; \# $\mathrm{P}<0.01$, $\mathrm{n} \geq 3$, and all values are represented as mean \pm SEM. Adapted from Yulia et al., Proceedings of National Academy of Science, 2014 [286]. 
A SVF isolation from human subcutaneous adipose tissue
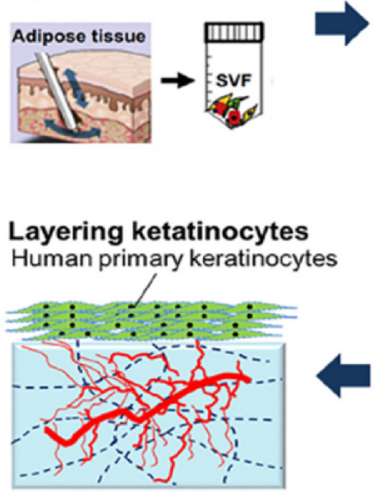

SVF cell encapsulation in fibrin hydrogel

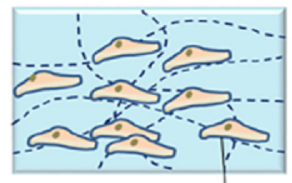

SVF cells

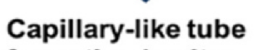

formation in vitro

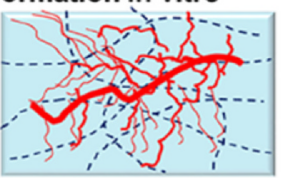

B

(i) Angiogenic ingrowth

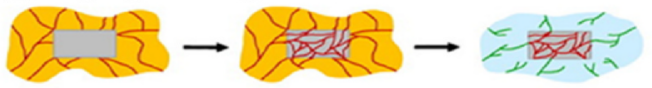

(ii) Flap technique

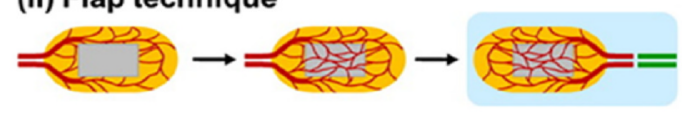

(iii) AV-loop technique

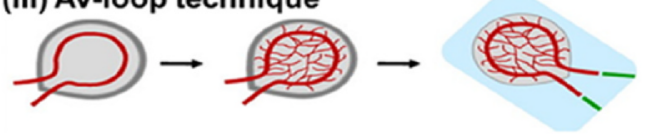

C Fabricate nanograted PDMS by nanoimprinting

\section{Seed hMSC on nanograted PDMS}

Culture hMSC to confluency in low $\mathrm{O}_{2}$ chamber for 2 weeks

\section{Harvest and wrap hMSC sheet around a mandrel circumferentially}
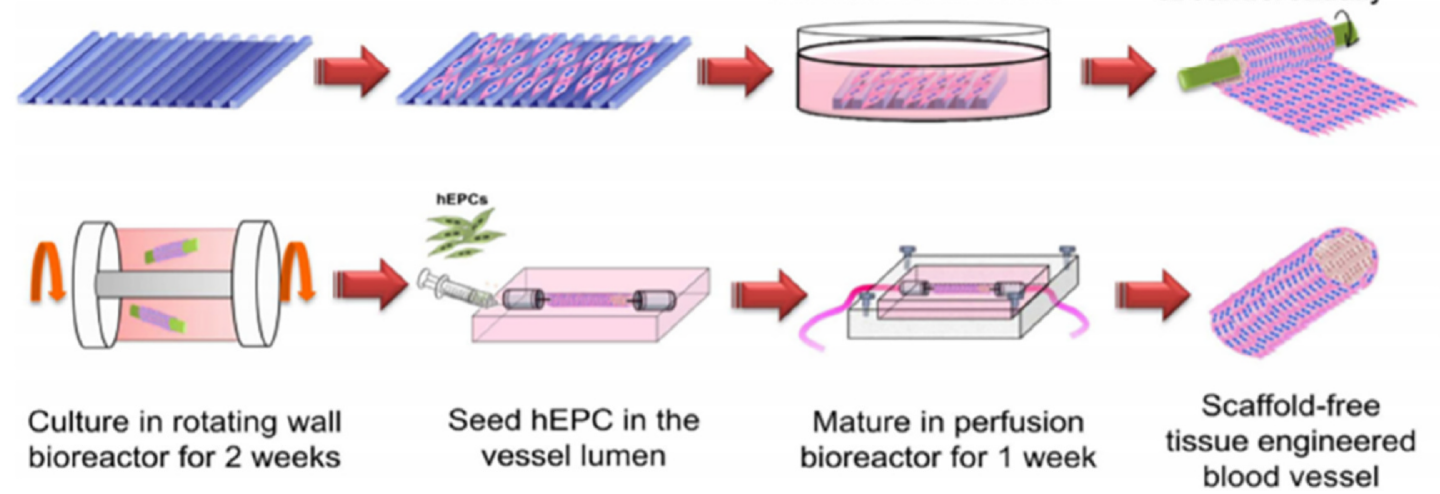

Fig. 32. Key prevascularization technologies based on cell constructs and tissue engineering (A) Delivery of tube forming cells in hydrogels for prevascularization by tube forming components (SVF: stromal vascular fraction). (B) In vivo skin bioreactor: (i) Ingrowth of newly developing microvessels in the scaffold, (ii) Use of scaffold-muscle flap complex including vein and vessels, and (iii) scaffold-spontaneous sprouting of vessels by AV-loop technique. After complete vascularization, the implanted biomaterial complex is transferred to the recipient site (blue), where the preformed microvasculatures inside the implant finally are interconnected to the microvessels of the recipient tissue (green) by inosculation. (C) Formation of scaffold-free tissue engineered blood vessel (TEBV) using hMSC and hEPC in perfusion system. Adapted from Kim et al., Adv Drug Deliv Res., 2018 [34]. (For interpretation of the references to color in this figure legend, the reader is referred to the web version of this article.)

\subsection{Biomaterials technologies for prevascularized cellular constructs and tissues}

Over the past years, technologies utilizing biomaterials have been advanced to generate cellular constructs and tissues that are prevascularized to connect host vasculature and thus can generate tissue equivalents. The need for prevascularized constructs arises from the fact that the scaffolded cells implanted in vivo are denied of nutrients to the innermost core owing to the slow diffusion rate of oxygen and growth rate of angiogenic neocapillaries. In fact, only cells in the vicinity of 100-200 $\mu \mathrm{m}$ from the nearest blood vessel get fed with nutrients and oxygen while the rest starves to death. Hence, a prevascularized construct can quickly deliver nutrients and oxygen to the innermost core by anastomozing with the host blood vessels (Fig. 32) [34,290].

Therefore, several key prevascularization technologies based on biomaterials have been developed. Tissue engineering bioreactor for prevascularization has been utilized; tubular perfusion assembly bioreactor, consisting of peristaltic pump to pump media from media reservoir to growth chamber [291] and rotating wall vessel bioreactor [292]. As another approach, biomaterial scaffolds were used to combine cell sheets to create a prevascularized structure. While the cell sheet technology has been demonstrated to be effective in neo-vasculature formation due mainly to high density cells and intimate cellular communications, it is basically $2 \mathrm{D}$ structure and thus its 3D-dimensionality mostly depends on the scaffold architecture used to support the cell sheets. One exemplar study designed porous cylindrical calcium phosphate ( $\beta$-TCP) scaffold to produce axially vascularized bone that can anastomose with the recipient vessel to initiate rapid blood flow to tissues. The rabbit MSC sheet combined with the scaffold and arteriovenous loop was shown to prefabricate axially vascularized construct which further facilitated new bone formation [293]. The biomaterialcombined cell sheet technology was also employed to improve osseointegration in irradiated bone as the radiotherapy is often known 

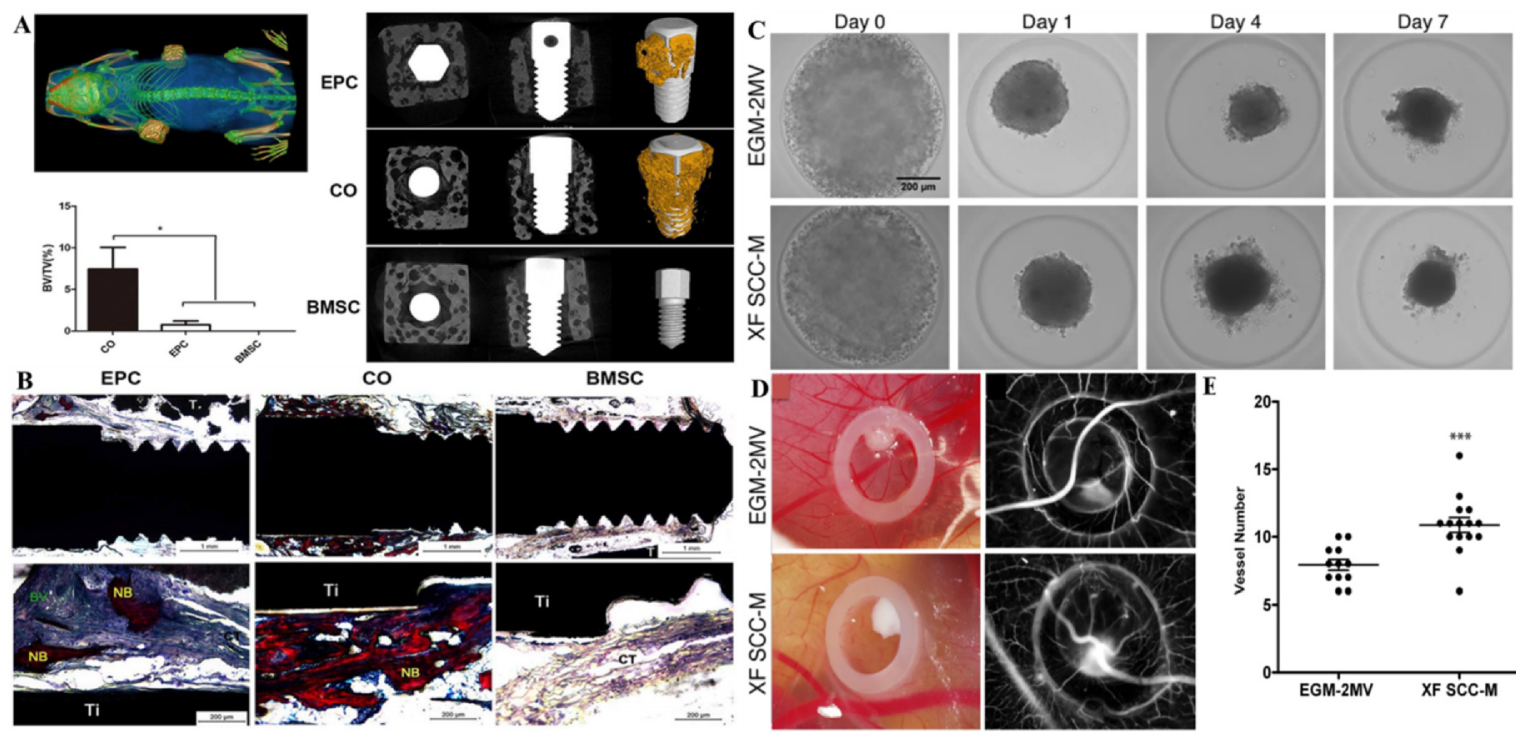

Fig. 33. Technologies for prevascularized cellular constructs. (A-B) EPC/BMSC co-cultured cell sheet wrapped around titanium implant for osteogenesis; (A) cell sheet-implant complex is embedded into the $\beta$-TCP cube for subcutaneous osteogenesis in nude mice. 3D reconstruction of the EPC, co-cultured (CO) and BMSC group, and (B) histologic sections showed increase of bone volume to total volume ration (BV/TV) in co-cultured group. Adapted from Liu et al., Sci Rep, 2017 [294]. (C-E) 3D MSC-outgrowth endothelial cell (OEC) spheroids under xenofree conditions; (C) morphology of MSC-OEC spheroids over 7-day culture. (D) angiogenic potential of spheroids tested using chick embryonic membrane, and (E) increase of vessel number in xenofree condition. Adapted from Bauman et al., Sci Rep, 2018 [295].

to result in significant failure of bone and dental implants (Fig. 33A-B) [294]. The co-cultured EPCs and MSCs sheet around titanium implants could create a cell sheet-implant complex. This cell-sheeted implant showed an excellent osteogenic differentiation around titanium in vitro and an increased neovascularization, demonstrating an advanced technique for osseointegration of the implants in irradiated bone. Although those studies of using biomaterials with cell sheets have shown some effects on prevascularization of the cellular constructs, the role of scaffolds is largely limited, i.e., combining sheeted cells in 3-dimensinality, rather than providing the matrix-stimulated cues to cells during sheet formation, which remains further area of interest to tune the cell sheet performance including prevascularization. Another technology is to prepare 3D MSC-OEC spheroids under xenofree conditions and test their angiogenic potential (Fig. 33C-E) [295]. These prevascularized spheroids maintained their phenotype and function in presence of supplement for cell culture and were able to produce vascular-like structure in xenofree medium under in vitro and in vivo conditions.

The 3D bioprinting technology offers great promise to create on-demand 3D architectures of prevascularized cellular constructs. 3D printing of biomaterials with cells has recently been given utmost attention as it can generate complex tissue architectures by a precise layer-by-layer positioning with a spatial control of biochemicals with living cells [28,296-297]. While many organs and tissues including bone, cartilage, cardiac tissue, skin, vessel, and nerve are currently 3D-mimicked by means of different printing methods [298], several key issues are still challenging; i) how to compose optimal biomaterial solution that is printable and cellcarriable (namely bioink) and ii) how to achieve fully vascularized structure. For instance, alginate, agarose, collagen, hyaluronan, silk fibroin, and decellularized matrix components have been engineered to gain proper bioink properties [299-302]. More information on the printable bioinks can be referenced from recent reviews [303-305], and here we focus on the efforts of 3D printing vasculatures and bone tissues.

Typically, disposable filaments with a vasculature structure were often 3D printed in a hydrogel matrix. For example, soluble sugar filaments were printed into biodegradable fibrin hydrogel and then dissolved off to create channels through which ECs could form networks (Fig. 34) [306]. When introduced into mouse hind limb ischemia the engineered vessels were shown to anastomose and integrate with the host vasculature which ultimately rescued significant perfusion of distal ischemic tissue. Among other bioinks, tissue-derived decellularized matrix has gained a lot attention due to the tissue mimic composition and microstructure. For example, pre-vascularized skin patch was generated using skin-derived extracellular matrix bioink [307]. The EPCs and adipose stem cells were mixed with the ECM bioink and then 3D-printed, and after culture for 3 days ex vivo, the skin tissue was implanted in a mouse dorsal wound healing model. Results showed enhanced neovascularization, robust blood flow and wound closure. Also, a minimal shrinkage was noticed in the engineered skin tissue which contrasted with the collagen skin tissue.

One challenging issue in current bioprinting techniques is the difficulty in achieving biomimetic nano-resolution and the incorporation of bioactive signals in a spatiotemporal way. Recently, biomimetic fluid perfused microstructures integrated with controlled release nanocoating system were achieved through 3D bioprinting for engineered vascularized bone construct [308]. Angiogenesis and osteogenesis were successively induced through a matrix metalloprotease- 2 regulative mechanism by delivering dual growth factors with sequential release in a spatiotemporal coordination. The nanocoating film was designed to load rhBMP-2 in the first 15 layers and then VEGF in the top 5 layers. When MSCs and HUVECs were co-cultured in a dynamic fluid, the HUVECs secreted 
A
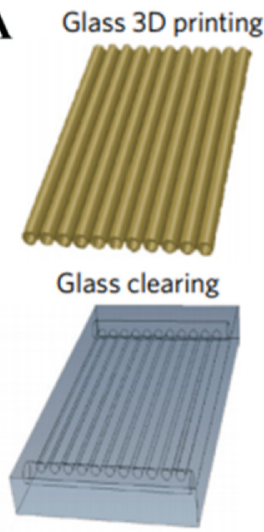

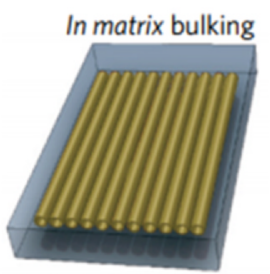

Endothelialization

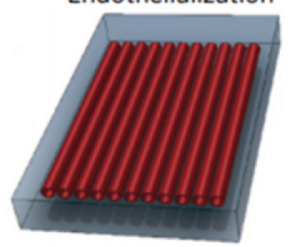

B
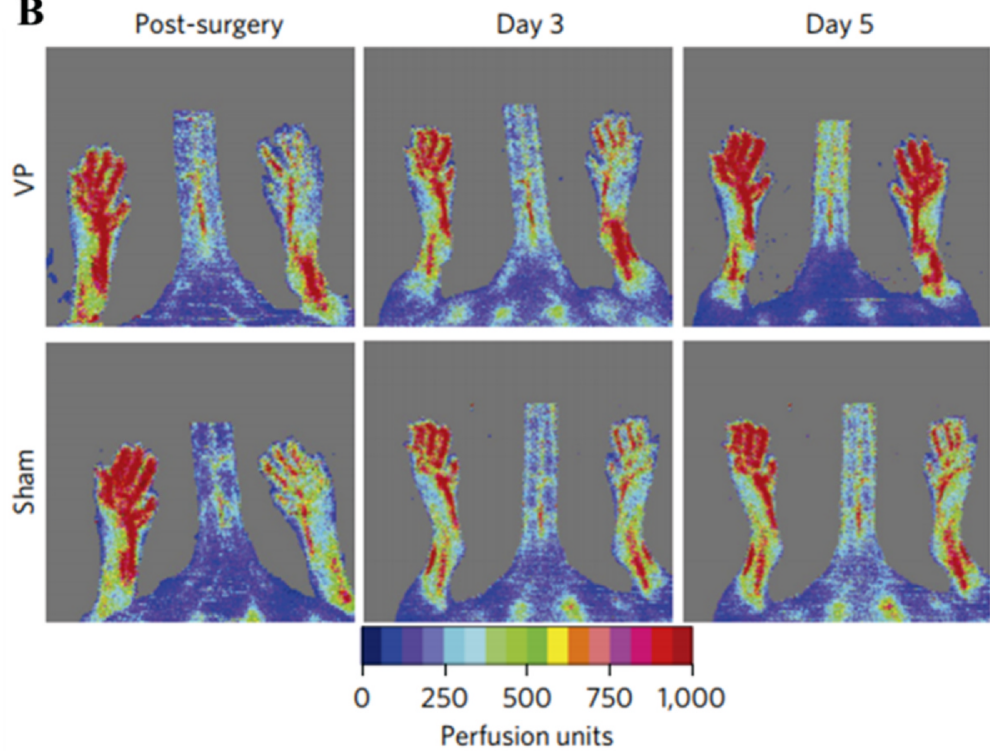

Implant in ischaemic model
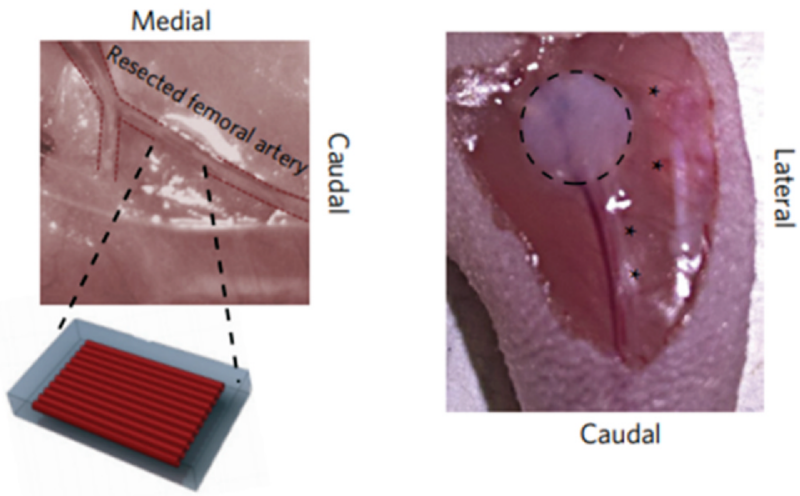

Caudal

Gross explants
Day 5
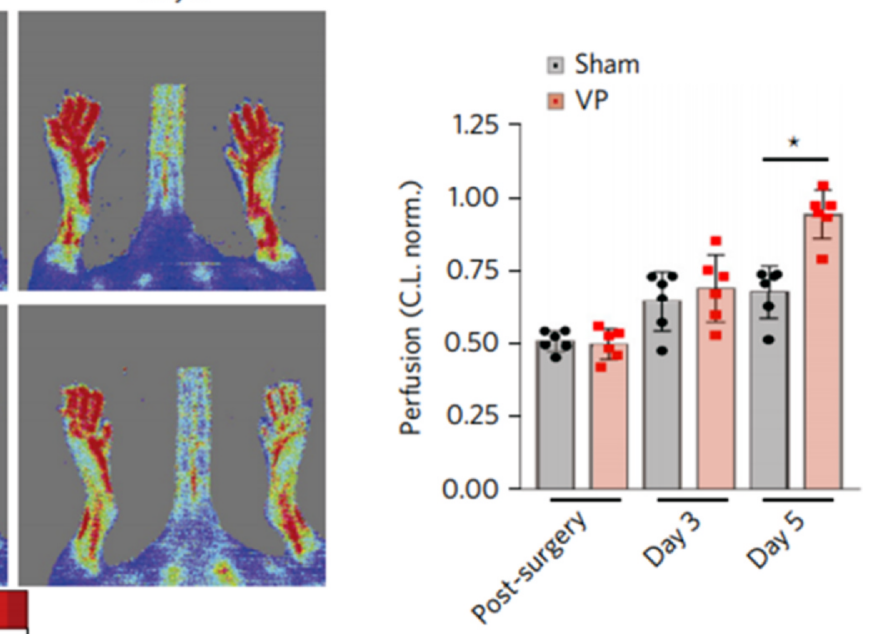

Fig. 34. 3D-printed vascular patch filled with HUVECs for revascularization of ischemic hind limb. Sugar filaments were 3D printed into a network and fibrin gel was encapsulated into the network. After dissolving the sugar to leave behind channels, HUVECs were injected to fill the channels and implanted into ischemic model. (B) In mice implanted at the injury site with above vascular patches (VPs), perfusion progressively increased over time, to reach the value of the contralateral, non-ischemic limb at 5 days, as measured by laser Doppler imaging. In the sham group, perfusion did not improve from the postoperative levels. Data are expressed as mean \pm s.d.; $\mathrm{N}=6$ animals per group; *P $<0.01$. $t$-test. Adapted from Mirabella et al., Nat Biomed Eng, 2017 [306].

MMP2 and then triggered the growth factor release. After 4 weeks of culture, a fully vascularized bone structure was formed in vitro. In another work, composite hydrogels with two-channels were prepared by 3D-bioprinting to enable precise cell loading and high viability [309]. The PEG-Clay prehydrogel solution was first introduced into one channel which allows the supply of oxygen and nutrients for cell growth, and after this primary rat osteoblast encapsulated in $20 \%$ hyaluronic acid (HA) solution was introduced to the other channel that can position cells accurately in 3D printed architecture. From in vivo studies, the cell-laden scaffolds exhibited improved osteogenesis owing to the conducive environment provided by the PEG-Clay scaffolds.

Patient-specific treatment of large bone defects with sufficient vascularization has been a significant challenge in clinics and the 3D bioprinting technology is considered to tackle this issue. However, bone is more rigid and robust compared with other soft tissues, so the use of strong supportive scaffolds along with cell-containing hydrogel-based bioink is often preferred. Some recent exemplar studies have utilized this approach of using supportive scaffold together with cell-hydrogel to vascularize bone constructs. In fact, recapitulation of the endochondral ossification process where mesenchymal cells condense to become hypertrophic chondrocytes and produce mineralized matrix that attract blood vessels has been a promising approach [310-311]. However, a problem is that the vascularization proceeds from the peripheral regions leaving the central region avascularized. To mitigate this, hypertrophic cartilage templates made of UV-curable gelatin methacrylate (GelMA) were 3D-printed to guide vascularization during endochondral bone repair (Fig. 35) [312]. Moreover, a sacrificial Pluronic ink (F127) was used to create microchannels in the hydrogels. The microchanneled hydrogels could induce bone vascularization at 4 weeks of implantation. To improve the bioink properties through 

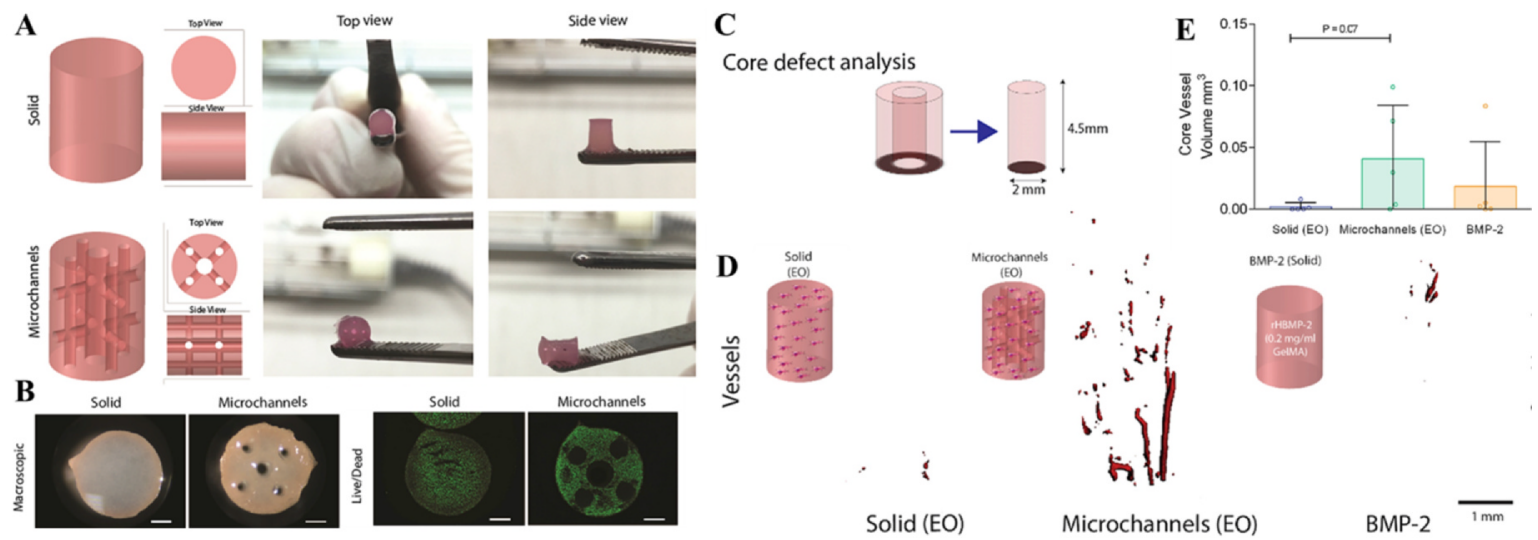

Fig. 35. Microchanneled 3D printed MSC-hydrogel for vascular ingrowth and anastomosis. (A) A sacrificial pluronic ink was used to 3D print interconnected microchannel networks in a MSC laden gelatin-methacryloyl (GelMA) hydrogel and then sacrificed by cooling the constructs in $4{ }^{\circ} \mathrm{C}$ media for $5 \mathrm{~min}$. Outline of construct design (solid and microchanneled) with top and side views. (B) Macroscopic images of constructs and live/ dead staining of MSC after $24 \mathrm{~h}$ fabrication, scale-bar $500 \mu \mathrm{m}$. (C) Schematic images of constructs for core defect analysis. (D) Representative 3D reconstructions of vessels within the defect core $(2 \mathrm{~mm} \emptyset$ ) from three different groups (E) Quantification of total vessel volume within the core of the defect. All graphs $\mathrm{n}=5$ (mean \pm SD), significance defined as $\mathrm{p}<0.05$ (ANOVA). Adapted from Daly et al., Biomaterials, 2018 [312].

crosslink methods, the GelMA combined with alginate and PEG tetra acrylate was used where the ionic- and photo-crosslinking process was selectively applied to generate GelMA-cells/alginate core/shell coaxial 3D printing [313]. The same group further extended the work to bioprinted patterns for osteogenesis and vascular morphogenesis by ultimately engineering a vascularized 3D printed bone tissue [314]. For this, GelMA was first 3D printed by a direct writing technique to create perfusable vascular lumen-like constructs, and then cell-laden cylindrical elements with GelMA/silicate nanoplatelets were introduced to initiate osteogenesis. The combined system led to a robust 3D bone tissue construct with functional vasculature, ready to replace large tissue bone defects.

As witnessed, the 3D bioprinting technique opens a promising avenue to generate fully vascularized large scale tissues with complex architectures. However, still hurdles remain as to the direct 3D bioprinting with cells mainly due to the lack of proper bioinks that are strong enough to mimic bone ECM while friendly to cells and sufficiently 3D-printable. Alternatively, and suboptimal though, strong and rigid 3D printable scaffolds are currently used as a support for the weak cellular constructs. Therefore, more attention is needed towards the supportive scaffold properties, such as printed 3D architecture, degradability and surface structure which can eventually guide and stimulate cells encapsulated in hydrogels. We have already discussed in the earlier section about how these properties determine the fate of cells in angiogenesis. Further, '4D bioprinting' has recently emerged by combining 3D bioprinting with an added fourth dimension of time. Here, 'time' refers to the duration of a 3D biocompatible material to continuously evolve over time [315]. Therefore, future studies may need to integrate the concepts established in biomaterials and scaffolds with the emerging 3D bioprinting technologies to create patient-specific prevascularized large tissue constructs.

\section{Concluding remarks}

Angiogenesis is a key event in the repair of tissues, supporting the delivery of nutrients and signaling factors and the removal of wastes, thus promoting neo-tissue formation. Biomaterials used to repair damaged tissue thus need to be designed to benefit from their critical actions in angiogenesis. Our thorough literature survey categorizes the biomaterials-based strategies to promoting angiogenesis mainly into three: i) utilization of their innate properties, i.e., tailoring pore structure and physico-chemical properties that are favorable for the angiogenic responses; ii) delivery through biomaterials of the exogenous factors effective in angiogenic events, particularly growth factors and metallic ions; iii) use of biomaterial scaffolds to cultivate cells (single or multiple cells) and to facilitate their organization into tissue-equivalent constructs.

A variety of biomaterials types and physico-chemical parameters have significant influences on the angiogenic events. However, the control of individual parameters in 3D biomaterials is still technically challenging; i.e., surface topographical control while preserving 3D dimensionality, and decoupling of stiffness and porosity in 3D scaffolds, needing further advances in biomaterials nano/micro-technologies. One thing to note is that fine-control of pore structure in 3D scaffolds by decoupling the effects of pore size, porosity and interconnectivity is currently possible in part with the help of 3D printing technology. Among other forms of biomaterials such as preformed 3D scaffolds, the hydrogels, due to their biomechanical properties that mimic native tissue matrix, can provide excellent 3D models allowing better interpretation of the in vivo angiogenesis mechanisms. In this case, dynamic properties of hydrogels such as stress relaxation and degradation should be considered as an important regulator of cell fate over time in angiogenesis and tissue repair.

Delivery of angiogenic molecules through biomaterials has been a potential strategy to promote angiogenesis. A set of growth factors that are known to be critically involved in angiogenesis has been widely used in conjunction with biomaterials. Biomaterials can preserve the activity of those proteins and release them controllably. Among other ways to link proteins to biomaterials, 
molecular affinity is considered promising in terms of preserving the activity while enabling presentation of functional domains, which needs a designer matrix that allows affinity binding with specific protein sequence. In fact, when growth factors are bound to biomaterials their receptor presentation and down-stream signaling in cells become much stronger. In this sense, biomaterials are often designed to conjugate growth factors and integrins to co-signal and synergize the angiogenic process. On the other stream, metallic ions have shown profound effects on angiogenesis and the related tissue repair process. Merited is the ease of incorporation of various ions into the composition of biomaterials. Moreover, ions have often been influential on other biological events such as inflammation and infection, thus been used to combat against inflammatory or infectious cells, which is a prerequisite in repair process of inflamed and infected tissues, implying possible drug-free therapeutics with the help of ions. In this sense, combined or time-sequential delivery of growth factors with ions can assist in augmenting angiogenesis and ultimately tissue repair.

In the angiogenesis-stimulated tissue regeneration, the cellular crosstalks are important; thus, many co-culture models to promote angiogenesis have been investigated. Biomaterials provide unique substrates to the co-cultured cells whereby the various sets of cellmatrix interactions influence the cellular crosstalks; i.e., surface properties and 3-dimensionality affect the inter-cellular signaling. For instance, the rapid cell aggregation in 3D scaffolding matrix boosts the crosstalks between ECs and MSCs required for vascularized tissue formation. Dynamic cultures also allow cells to communicate under in vivo mimic conditions, and the combination with 3D biomaterials can be further advanced to create prevascularized cellular constructs that possibly connect host vasculature and generate tissue equivalents suitable for large scale defects. Well-designed bioreactors and cell sheet engineering are some of the representative examples for the prevascularized structures. Among others technologies, the development of 3D bioprinting advances the possibility of prevascularized cellular constructs with complex architectures. Although many hurdles remain as to the success of the 3D bioprinting, such as the choice of bioinks that can properly incorporate cells and signaling molecules with robust physical properties, some of the recent pioneering studies have shown highly developed vasculature architectures by the 3D bioprinting techniques.

As discussed, a lot of efforts have been made to stimulate tissue angiogenesis through biomaterials approach - the control of pore architecture and physicochemical properties, the delivery of angiogenic molecules and ions, and the prevascularization of tissue constructs. In fact, the biomaterial-enabled pro-angiogenic event and vasculature formation have recently been significantly improved by the development of novel biomaterials, the design of controlled delivery systems, and the advent of 3D bioprinting technology. However, challenging issues still remain to be solved. For example, the matrix roles in different cellular interactions during angiogenesis are not fully undermined. Also, the spatiotemporal control of delivery molecules is needed to better recapitulate the synergized effects of multiple angiogenic factors for tissue regeneration. Furthermore, future technological advances in creating fully vascularized constructs of large scale tissues may hopefully satisfy the clinically unmet need.

\section{Declaration of Competing Interest}

The authors declare that they have no known competing financial interests or personal relationships that could have appeared to influence the work reported in this paper.

\section{Acknowledgements}

This study was supported by grants from the National Research Foundation (NRF), Republic of Korea (Global Research Lab Program 20150093829; NRF-2018R1A2B3003446; NRF-2018K1A4A3A01064257; 2019R1A6A1A11034536; NRF2019R1C1C1002490).

\section{References}

[1] Grimes DR, Kelly C, Bloch K, Partridge M. A method for estimating the oxygen consumption rate in multicellular tumour spheroids. J R Soc Interface 2014;11(92):20131124.

[2] Thomlinson RH, Gray LH. The histological structure of some human lung cancers and the possible implications for radiotherapy. Br J Cancer 1955;9(4):539-49.

[3] Lee S-I, Lee E-S, El-Fiqi A, Lee S-Y, Kim E-C, Kim H-W. Stimulation of odontogenesis and angiogenesis via bioactive nanocomposite calcium phosphate cements through integrin and VEGF signaling pathways. J Biomed Nanotechnol 2016;12(5):1048-62.

[4] Nannan D, Xue G, Lin Y, Aiying Z, Zengguo F, Lianrui G, et al. A vascular tissue engineering scaffold with core-shell structured nano-fibers formed by coaxial electrospinning and its biocompatibility evaluation. Biomed Mater 2016;11(3):035007.

[5] Stegen S, van Gastel N, Carmeliet G. Bringing new life to damaged bone: the importance of angiogenesis in bone repair and regeneration. Bone 2015;70:19-27.

[6] Mukouyama Y-S, Shin D, Britsch S, Taniguchi M, Anderson DJ. Sensory nerves determine the pattern of arterial differentiation and blood vessel branching in the skin. Cell 2002;109(6):693-705.

[7] Veith AP, Henderson K, Spencer A, Sligar AD, Baker AB. Therapeutic strategies for enhancing angiogenesis in wound healing. Adv Drug Deliv Rev 2018.

[8] Carmeliet P. Blood vessels and nerves: common signals, pathways and diseases. Nat Rev Genet 2003;4:710.

[9] Song YH, Shon SH, Shan M, Stroock AD, Fischbach C. Adipose-derived stem cells increase angiogenesis through matrix metalloproteinase-dependent collagen remodeling. Integr Biol 2016;8(2):205-15.

[10] Risau W. Angiogenesis is coming of age. Circ Res 1998;82(8):926-8.

[11] Risau W, Flamme I. Vasculogenesis. Annu Rev Cell Dev Biol 1995;11(1):73-91.

[12] Rouwkema J, Khademhosseini A. Vascularization and angiogenesis in tissue engineering: beyond creating static networks. Trends Biotechnol 2016;34(9):733-45.

[13] Bryan BA, D'Amore PA. What tangled webs they weave: Rho-GTPase control of angiogenesis. Cell Mol Life Sci 2007;64:2053-65.

[14] Risau W. Mechanisms of angiogenesis. Nature 1997;386(6626):671-4.

[15] Fuchs S, Ghanaati S, Orth C, Barbeck M, Kolbe M, Hofmann A, et al. Contribution of outgrowth endothelial cells from human peripheral blood on in vivo vascularization of bone tissue engineered constructs based on starch polycaprolactone scaffolds. Biomaterials 2009;30(4):526-34.

[16] Augustin HG, Young Koh G, Thurston G, Alitalo K. Control of vascular morphogenesis and homeostasis through the angiopoietin-Tie system. Nat Rev Mol Cell 
Biol 2009;10(3):165-77.

[17] Shah AV, Birdsey GM, Randi AM. Regulation of endothelial homeostasis, vascular development and angiogenesis by the transcription factor ERG. VascPharmacol 2016;86:3-13.

[18] Morland C, Andersson KA, Haugen ØP, Hadzic A, Kleppa L, Gille A, et al. Exercise induces cerebral VEGF and angiogenesis via the lactate receptor HCAR1. Nat Commun 2017;8:15557.

[19] Stevens MM. Biomaterials for bone tissue engineering. Mater Today 2008;11(5):18-25.

[20] Haycock JW, Unger R, Halstenberg S, Sartoris A, Kirkpatrick CJ. Human endothelial and osteoblast co-cultures on 3D biomaterials, 3D cell culture. Humana Press; 2011. p. 229-41.

[21] Jin Woo L, Yeong-Jin C, Woon-Jae Y, Falguni P, Jin-Hyung S, Kyung Shin K, et al. Development of a 3D cell printed construct considering angiogenesis for liver tissue engineering. Biofabrication 2016;8(1):015007.

[22] Xiao X, Wang W, Liu D, Zhang H, Gao P, Geng L, et al. The promotion of angiogenesis induced by three-dimensional porous beta-tricalcium phosphate scaffold with different interconnection sizes via activation of PI3K/Akt pathways. Sci Rep 2015;5:9409.

[23] Baino F, Novajra G, Vitale-Brovarone C. Bioceramics and scaffolds: a winning combination for tissue engineering. Front Bioeng Biotechnol 2015;3.

[24] Tan AW, Liau LL, Chua KH, Ahmad R, Akbar SA, Pingguan-Murphy B. Enhanced in vitro angiogenic behaviour of human umbilical vein endothelial cells on thermally oxidized TiO(2) nanofibrous surfaces. Sci Rep 2016;6:21828.

[25] Shi B, Andrukhov O, Berner S, Schedle A, Rausch-Fan X. The angiogenic behaviors of human umbilical vein endothelial cells (HUVEC) in co-culture with osteoblast-like cells (MG-63) on different titanium surfaces. Dent Mater 2014;30(8):839-47.

[26] Li J, Zhi W, Xu T, Shi F, Duan K, Wang J, et al. Ectopic osteogenesis and angiogenesis regulated by porous architecture of hydroxyapatite scaffolds with similar interconnecting structure in vivo. Regener Biomater 2016;3(5):285-97.

[27] Wu XJ, Bruschi M, Waag T, Schweeberg S, Tian Y, Meinhardt T, et al. Functionalization of bone implants with nanodiamond particles and angiopoietin-1 to improve vascularization and bone regeneration. J Mater Chem B 2017;5(32):6629-36.

[28] Sun J, Wang Y, Qian Z, Hu C. An approach to architecture 3D scaffold with interconnective microchannel networks inducing angiogenesis for tissue engineering. J Mater Sci - Mater Med 2011;22(11):2565-71.

[29] Mehdizadeh H, Bayrak ES, Lu C, Somo SI, Akar B, Brey EM, et al. Agent-based modeling of porous scaffold degradation and vascularization: optimal scaffold design based on architecture and degradation dynamics. Acta Biomater 2015;27:167-78.

[30] Metters AT, Bowman CN, Anseth KS. A statistical kinetic model for the bulk degradation of PLA-b-PEG-b-PLA hydrogel networks. J Phys Chem B 2000;104(30):7043-9.

[31] Sun X, Kang Y, Bao J, Zhang Y, Yang Y, Zhou X. Modeling vascularized bone regeneration within a porous biodegradable CaP scaffold loaded with growth factors. Biomaterials 2013;34(21):4971-81.

[32] Klenke FM, Liu Y, Yuan H, Hunziker EB, Siebenrock KA, Hofstetter W. Impact of pore size on the vascularization and osseointegration of ceramic bone substitutes in vivo. J Biomed Mater Res Part A 2008;85A(3):777-86.

[33] Kang Y, Mochizuki N, Khademhosseini A, Fukuda J, Yang Y. Engineering a vascularized collagen- $\beta$-tricalcium phosphate graft using an electrochemical approach. Acta Biomater 2015;11:449-58.

[34] Kim HS, Sun X, Lee J-H, Kim H-W, Fu X, Leong KW. Advanced drug delivery systems and artificial skin grafts for skin wound healing. Adv Drug Deliv Rev 2018.

[35] Lee J-H, Kim H-W. Emerging properties of hydrogels in tissue engineering. J Tissue Eng 2018;9. 2041731418768285.

[36] Oliviero O, Ventre M, Netti PA. Functional porous hydrogels to study angiogenesis under the effect of controlled release of vascular endothelial growth factor. Acta Biomater 2012;8(9):3294-301.

[37] Chiu Y-C, Cheng M-H, Engel H, Kao S-W, Larson JC, Gupta S, et al. The role of pore size on vascularization and tissue remodeling in PEG hydrogels. Biomaterials 2011;32(26):6045-51.

[38] Tokatlian T, Cam C, Segura T. Porous hyaluronic acid hydrogels for localized nonviral DNA delivery in a diabetic wound healing model. Adv Healthcare Mater 2015;4(7):1084-91.

[39] Loh QL, Choong C. Three-dimensional scaffolds for tissue engineering applications: role of porosity and pore size. Tissue Eng Part B: Rev 2013;19(6):485-502.

[40] Liao H-T, Chen Y-Y, Lai Y-T, Hsieh M-F, Jiang C-P. The osteogenesis of bone marrow stem cells on mPEG-PCL-mPEG/hydroxyapatite composite scaffold via solid freeform fabrication. Biomed Res Int 2014;2014:13.

[41] Joshi VS, Lei NY, Walthers CM, Wu B, Dunn JCY. Macroporosity enhances vascularization of electrospun scaffolds. J Surg Res 2013;183(1):18-26.

[42] Nam J, Huang Y, Agarwal S, Lannutti J. Improved cellular infiltration in electrospun fiber via engineered porosity. Tissue Eng 2007;13(9):2249-57.

[43] Wang Z, Cui Y, Wang J, Yang X, Wu Y, Wang K, et al. The effect of thick fibers and large pores of electrospun poly(e-caprolactone) vascular grafts on macrophage polarization and arterial regeneration. Biomaterials 2014;35(22):5700-10.

[44] Walthers CM, Nazemi AK, Patel SL, Wu BM, Dunn JCY. The effect of scaffold macroporosity on angiogenesis and cell survival in tissue-engineered smooth muscle. Biomaterials 2014;35(19):5129-37.

[46] Somo SI, Akar B, Bayrak ES, Larson JC, Appel AA, Mehdizadeh H, et al. Pore interconnectivity influences growth factor-mediated vascularization in spheretemplated hydrogels. Tissue Eng Part C: Methods 2015;21(8):785.

[47] Martino MM, Tortelli F, Mochizuki M, Traub S, Ben-David D, Kuhn GA, et al. Engineering the growth factor microenvironment with fibronectin domains to promote wound and bone tissue healing. Sci Transl Med 2011;3(100):100ra89.

[48] Vournakis JN, Eldridge J, Demcheva M, Muise-Helmericks RC. Poly-N-acetyl glucosamine nanofibers regulate endothelial cell movement and angiogenesis: dependency on integrin activation of ets1. J Vasc Res 2008;45(3):222-32.

[49] Castellanos Maria I, Guillem-Marti J, Mas-Moruno C, Díaz-Ricart M, Escolar G, Ginebra Maria P, et al. Cell adhesive peptides functionalized on CoCr alloy stimulate endothelialization and prevent thrombogenesis and restenosis. J Biomed Mater Res Part A 2017;105(4):973-83.

[50] Sakiyama-Elbert SE. Incorporation of heparin into biomaterials. Acta Biomater 2014;10(4):1581-7.

[51] Singh S, Wu BM, Dunn JCY. The enhancement of VEGF-mediated angiogenesis by polycaprolactone scaffolds with surface cross-linked heparin. Biomaterials 2011;32(8):2059-69.

[52] Melchiorri AJ, Hibino N, Yi T, Lee YU, Sugiura T, Tara S, et al. Contrasting biofunctionalization strategies for the enhanced endothelialization of biodegradable vascular grafts. Biomacromolecules 2015;16(2):437-46.

[53] Quade M, Knaack S, Weber D, König U, Paul B, Simon P, et al. Heparin modification of a biomimetic bone matrix modulates osteogenic and angiogenic cell response in vitro. Eur Cell Mater 2017:105-20.

[54] Lee MH, Ducheyne P, Lynch L, Boettiger D, Composto RJ. Effect of biomaterial surface properties on fibronectin- $\alpha 5 \beta 1$ integrin interaction and cellular attachment. Biomaterials 2006;27(9):1907-16.

[55] Slater John H, Frey W. Nanopatterning of fibronectin and the influence of integrin clustering on endothelial cell spreading and proliferation. J Biomed Mater Res Part A 2007;87A(1):176-95.

[56] Moulisová V, Gonzalez-García C, Cantini M, Rodrigo-Navarro A, Weaver J, Costell M, et al. Engineered microenvironments for synergistic VEGF - Integrin signalling during vascularization. Biomaterials 2017;126:61-74.

[57] Lim JY, Dreiss AD, Zhou Z, Hansen JC, Siedlecki CA, Hengstebeck RW, et al. The regulation of integrin-mediated osteoblast focal adhesion and focal adhesion kinase expression by nanoscale topography. Biomaterials 2007;28(10):1787-97.

[58] Dobbenga S, Fratila-Apachitei LE, Zadpoor AA. Nanopattern-induced osteogenic differentiation of stem cells - A systematic review. Acta Biomater 2016;46:3-14.

[59] Bettinger CJ, Zhang Z, Gerecht S, Borenstein JT, Langer R. Enhancement of in vitro capillary tube formation by substrate nanotopography. Adv Mater 2008;20(1):99-103.

[60] Kim TH, Kim SH, Jung Y. The effects of nanotopography and coculture systems to promote angiogenesis for wound repair. Nanomedicine 2016;11(22):2997-3007. 
[61] Olivares-Navarrete R, Raz P, Zhao G, Chen J, Wieland M, Cochran DL, et al. Integrin a2ß1 plays a critical role in osteoblast response to micron-scale surface structure and surface energy of titanium substrates. Proc Natl Acad Sci 2008;105:15767-72.

[62] Olivares-Navarrete R, Rodil SE, Hyzy SL, Dunn GR, Almaguer-Flores A, Schwartz Z, et al. Role of integrin subunits in mesenchymal stem cell differentiation and osteoblast maturation on graphitic carbon-coated microstructured surfaces. Biomaterials 2015;51:69-79.

[63] Olivares-Navarrete R, Hyzy SL, Gittens RA, Schneider JM, Haithcock DA, Ullrich PF, et al. Rough titanium alloys regulate osteoblast production of angiogenic factors. The Spine Journal 2013;13(11):1563-70.

[64] Raines AL, Olivares-Navarrete R, Wieland M, Cochran DL, Schwartz Z, Boyan BD. Regulation of angiogenesis during osseointegration by titanium surface microstructure and energy. Biomaterials 2010;31(18):4909-17.

[65] Chung T-W, Liu D-Z, Wang S-Y, Wang S-S. Enhancement of the growth of human endothelial cells by surface roughness at nanometer scale. Biomaterials 2003;24(25):4655-61.

[66] Biela SA, Su Y, Spatz JP, Kemkemer R. Different sensitivity of human endothelial cells, smooth muscle cells and fibroblasts to topography in the nano-micro range. Acta Biomater 2009;5(7):2460-6.

[67] Uttayarat P, Toworfe GK, Dietrich F, Lelkes PI, Composto RJ. Topographic guidance of endothelial cells on silicone surfaces with micro- to nanogrooves: orientation of actin filaments and focal adhesions. J Biomed Mater Res Part A 2005;75A(3):668-80.

[68] Klumpp D, Rudisile M, Kühnle RI, Hess A, Bitto FF, Arkudas A, et al. Three-dimensional vascularization of electrospun PCL/collagen-blend nanofibrous scaffolds in vivo. J Biomed Mater Res A 2012;100A:2302-11.

[69] Kuo Cheng-Hwa R, Xian J, Brenton James D, Franze K, Sivaniah E. Complex stiffness gradient substrates for studying mechanotactic cell migration. Adv Mater 2012;24(45):6059-64.

[70] Choi B, Park KS, Kim JH, Ko KW, Kim JS, Han Dong K, et al. Stiffness of hydrogels regulates cellular reprogramming efficiency through mesenchymal-toepithelial transition and stemness markers. Macromol Biosci 2015;16(2):199-206.

[71] Mao AS, Shin J-W, Mooney DJ. Effects of substrate stiffness and cell-cell contact on mesenchymal stem cell differentiation. Biomaterials 2016;98:184-91.

[72] Discher DE, Janmey P, Wang Y-L. Tissue cells feel and respond to the stiffness of their substrate. Science 2005;310(5751):1139.

[73] Leipzig ND, Shoichet MS. The effect of substrate stiffness on adult neural stem cell behavior. Biomaterials 2009;30(36):6867-78.

[74] Evans ND, Minelli C, Gentleman E, LaPointe V, Patankar SN, Kallivretaki M, et al. Substrate stiffness affects early differentiation events in embryonic stem cells. Eur Cell Mater 2009;18:1-14.

[75] Schrader J, Gordon-Walker TT, Aucott RL, van Deemter M, Quaas A, Walsh S, et al. Matrix stiffness modulates proliferation, chemotherapeutic response, and dormancy in hepatocellular carcinoma cells. Hepatology 2011;53(4):1192-205.

[76] Byfield FJ, Reen RK, Shentu T-P, Levitan I, Gooch KJ. Endothelial actin and cell stiffness is modulated by substrate stiffness in 2D and 3D. J Biomech 2009;42(8):1114-9.

[77] Stevenson MD, Piristine H, Hogrebe NJ, Nocera TM, Boehm MW, Reen RK, et al. A self-assembling peptide matrix used to control stiffness and binding site density supports the formation of microvascular networks in three dimensions. Acta Biomater 2013;9(8):7651-61.

[78] Santos L, Fuhrmann G, Juenet M, Amdursky N, Horejs CM, Campagnolo P, et al. Extracellular stiffness modulates the expression of functional proteins and growth factors in endothelial cells. Adv Healthcare Mater 2015;4(14):2056-63.

[79] Sung HJ, Meredith C, Johnson C, Galis ZS. The effect of scaffold degradation rate on three-dimensional cell growth and angiogenesis. Biomaterials 2004;25(26):5735-42.

[80] Castaño O, Sachot N, Xuriguera E, Engel E, Planell JA, Park J-H, et al. Angiogenesis in bone regeneration: tailored calcium release in hybrid fibrous scaffolds. ACS Appl Mater Interfaces 2014;6(10):7512-22.

[81] Chen YW, Shi GQ, Ding YL, Yu XX, Zhang XH, Zhao CS, et al. In vitro study on the influence of strontium-doped calcium polyphosphate on the angiogenesisrelated behaviors of HUVECs. J Mater Sci - Mater Med 2008;19(7):2655-62.

[82] Shahram MG, Benjamin WT, Ronald EU, Carina O, Thomas K, Mike B, et al. Collagen-embedded hydroxylapatite-beta-tricalcium phosphate-silicon dioxide bone substitute granules assist rapid vascularization and promote cell growth. Biomed Mater 2010;5(2):025004.

[83] Velasco MA, Narv, xe, ez-Tovar CA, Garz, xf, n-Alvarado DA. Design, materials, and mechanobiology of biodegradable scaffolds for bone tissue engineering. BioMed Res Int 2015;2015:21.

[84] Bauer AL, Jackson TL, Jiang Y. Topography of extracellular matrix mediates vascular morphogenesis and migration speeds in angiogenesis. PLoS Comput Biol 2009;5(7):e1000445.

[85] Ehrbar M, Djonov VG, Schnell C, Tschanz SA, Martiny-Baron G, Schenk U, et al. Cell-demanded liberation of VEGF-121 from fibrin implants induces local and controlled blood vessel growth. Circ Res 2004;94(8):1124.

[86] Blache U, Metzger S, Vallmajo-Martin Q, Martin I, Djonov V, Ehrbar M. Dual role of mesenchymal stem cells allows for microvascularized bone tissue-like environments in PEG hydrogels. Adv Healthcare Mater 2015;5(4):489-98.

[87] Chaudhuri O, Gu L, Klumpers D, Darnell M, Bencherif SA, Weaver JC, et al. Hydrogels with tunable stress relaxation regulate stem cell fate and activity. Nat Mater 2016;15(3):326.

[88] Chaudhuri O, Gu L, Darnell M, Klumpers D, Bencherif SA, Weaver JC, et al. Substrate stress relaxation regulates cell spreading. Nat Commun 2015;6.

[89] Pérez RA, Won J-E, Knowles JC, Kim H-W. Naturally and synthetic smart composite biomaterials for tissue regeneration. Adv Drug Deliv Rev 2013;65(4):471-96.

[90] Hoppe A, Güldal NS, Boccaccini AR. A review of the biological response to ionic dissolution products from bioactive glasses and glass-ceramics. Biomaterials 2011;32(11):2757-74.

[91] Sen CK, Khanna S, Venojarvi M, Trikha P, Ellison EC, Hunt TK, et al. Copper-induced vascular endothelial growth factor expression and wound healing. Am J Physiol Heart Circul Physiol 2002;282(5):H1821-7.

[92] Dingsheng L, Zengbing L, Dong H. Favorable effects of progesterone on skin random flap survival in rats. Iran J Basic Med Sci 2016;19(11):1166-70.

[93] Hu GF. Copper stimulates proliferation of human endothelial cells under culture. J Cell Biochem 1998;69(3):326-35.

[94] Finney L, Vogt S, Fukai T, Glesne D. Copper and angiogenesis: unravelling a relationship key to cancer progression. Clin Exp Pharmacol Physiol 2009;36(1):88-94.

[95] Gremmels H, Teraa M, Quax PHA, den Ouden K, Fledderus JO, Verhaar MC. Neovascularization capacity of mesenchymal stromal cells from critical limb ischemia patients is equivalent to healthy controls. Mol Ther 2014;22(11):1960-70.

[96] Michiels C, Arnould T, Remacle J. Endothelial cell responses to hypoxia: initiation of a cascade of cellular interactions. Biochim Biophys Acta (BBA) - Mol Cell Res 2000;1497(1):1-10.

[97] Okuyama H, Krishnamachary B, Zhou YF, Nagasawa H, Bosch-Marce M, Semenza GL. Expression of vascular endothelial growth factor receptor 1 in bone marrow-derived mesenchymal cells is dependent on hypoxia-inducible factor 1. J Biol Chem 2006;281(22):15554-63.

[98] El Sayed Mohammed Youssef H, Eldeen Abo-Azma NE, Eldeen Megahed EM. Correlation of hypoxia-inducible factor-1 alpha (HIF-1 $\alpha$ ) and vascular endothelial growth factor (VEGF) expressions with clinico-pathological features of oral squamous cell carcinoma (OSCC). Tanta Dental J 2015;12(Supplement 1):S1-14.

[99] Hung S-C, Pochampally RR, Hsu S-C, Sanchez C, Chen S-C, Spees J, et al. Short-term exposure of multipotent stromal cells to low oxygen increases their expression of CX3CR1 and CXCR4 and their engraftment in vivo. PLoS ONE 2007;2(5):e416.

[100] Zhang Y, Huang J, Wang C, Zhang Y, Hu C, Li G, et al. Application of HIF-1 $\alpha$ by gene therapy enhances angiogenesis and osteogenesis in alveolar bone defect regeneration. J Gene Med 2016;18(4-6):57-64.

[101] Zou D, Han W, You S, Ye D, Wang L, Wang S, et al. In vitro study of enhanced osteogenesis induced by HIF-1 $\alpha$-transduced bone marrow stem cells. Cell Prolif 2011;44(3):234-43.

[102] Liu H, Yang X, Zhang Y, Dighe A, Li X, Cui Q. Fullerol antagonizes dexamethasone-induced oxidative stress and adipogenesis while enhancing osteogenesis in a cloned bone marrow mesenchymal stem cell. J Orthop Res 2012;30(7):1051-7.

[103] Ding H, Gao Y-S, Hu C, Wang Y, Wang C-G, Yin J-M, et al. HIF-1 $\alpha$ transgenic bone marrow cells can promote tissue repair in cases of corticosteroid-induced 
osteonecrosis of the femoral head in rabbits. PLoS ONE 2013;8(5):e63628.

[104] Wu C, Zhou Y, Xu M, Han P, Chen L, Chang J, et al. Copper-containing mesoporous bioactive glass scaffolds with multifunctional properties of angiogenesis capacity, osteostimulation and antibacterial activity. Biomaterials 2013;34(2):422-33.

[105] Bari A, Bloise N, Fiorilli S, Novajra G, Vallet-Regí M, Bruni G, et al. Copper-containing mesoporous bioactive glass nanoparticles as multifunctional agent for bone regeneration. Acta Biomater 2017;55:493-504.

[106] Barralet J, Gbureck U, Habibovic P, Vorndran E, Gerard C, Doillon CJ. Angiogenesis in calcium phosphate scaffolds by inorganic copper ion release. Tissue Eng Part A 2009;15(7):1601-9.

[107] Fan W, Crawford R, Xiao Y. Enhancing in vivo vascularized bone formation by cobalt chloride-treated bone marrow stromal cells in a tissue engineered periosteum model. Biomaterials 2010;31(13):3580-9.

[108] Wu C, Zhou Y, Fan W, Han P, Chang J, Yuen J, et al. Hypoxia-mimicking mesoporous bioactive glass scaffolds with controllable cobalt ion release for bone tissue engineering. Biomaterials 2012;33(7):2076-85.

[109] Hoppe A, Jokic B, Janackovic D, Fey T, Greil P, Romeis S, et al. Cobalt-releasing 1393 bioactive glass-derived scaffolds for bone tissue engineering applications. ACS Appl Mater Interfaces 2014;6(4):2865-77.

[110] Quinlan E, Partap S, Azevedo MM, Jell G, Stevens MM, O'Brien FJ. Hypoxia-mimicking bioactive glass/collagen glycosaminoglycan composite scaffolds to enhance angiogenesis and bone repair. Biomaterials 2015;52:358-66.

[111] Zhang M, Wu C, Li H, Yuen J, Chang J, Xiao Y. Preparation, characterization and in vitro angiogenic capacity of cobalt substituted $\beta$-tricalcium phosphate ceramics. J Mater Chem 2012;22(40):21686-94.

[112] Boldbaatar K, Dashnyam K, Knowles JC, Lee H-H, Lee J-H, Kim H-W. Dual-ion delivery for synergistic angiogenesis and bactericidal capacity with silica-based microsphere. Acta Biomater 2019;83:322-33.

[113] Carlisle E. In vivo requirement for silicon in articular cartilage and connective tissue formation in the chick. J Nutr 1976;106:478-84.

[114] Li C, Jiang C, Deng Y, Li T, Li N, Peng M, et al. RhBMP-2 loaded 3D-printed mesoporous silica/calcium phosphate cement porous scaffolds with enhanced vascularization and osteogenesis properties. Sci Rep 2017;7:41331.

[115] Zhai W, Lu H, Chen L, Lin X, Huang Y, Dai K, et al. Silicate bioceramics induce angiogenesis during bone regeneration. Acta Biomater 2012;8(1):341-9.

[116] Dashnyam K, El-Fiqi A, Buitrago JO, Perez RA, Knowles JC, Kim H-W. A mini review focused on the proangiogenic role of silicate ions released from siliconcontaining biomaterials. J Tissue Eng 2017;8:1-13.

[117] Lin C, Mao C, Zhang J, Li Y, Chen X. Healing effect of bioactive glass ointment on full-thickness skin wounds. Biomed Mater (Bristol, England) 2012;7(4):045017.

[118] Yu H, Peng J, Xu Y, Chang J, Li H. Bioglass activated skin tissue engineering constructs for wound healing. ACS Appl Mater Interfaces 2016;8(1):703-15.

[119] Xavier JR, Thakur T, Desai P, Jaiswal MK, Sears N, Cosgriff-Hernandez E, et al. Bioactive nanoengineered hydrogels for bone tissue engineering: a growth factor-free approach. ACS Nano 2015;9(3):3109-18.

[120] Xia L, Yin Z, Mao L, Wang X, Liu J, Jiang X, et al. Akermanite bioceramics promote osteogenesis, angiogenesis and suppress osteoclastogenesis for osteoporotic bone regeneration. Sci Rep 2016;6:22005.

[121] Dashnyam K, Jin G-Z, Kim J-H, Perez R, Jang J-H, Kim H-W. Promoting angiogenesis with mesoporous microcarriers through a synergistic action of delivered silicon ion and VEGF. Biomaterials 2017;116:145-57.

[122] Ammann P. Strontium ranelate: a physiological approach for an improved bone quality. Bone 2006;38(2):15-8.

[123] Yang F, Yang DZ, Tu J, Zheng QX, Cai LT, Wang LP. Strontium enhances osteogenic differentiation of mesenchymal stem cells and in vivo bone formation by activating Wnt/catenin signaling. Stem Cells 2011;29(6):981-91.

[124] Lin K, Xia L, Li H, Jiang X, Pan H, Xu Y, et al. Enhanced osteoporotic bone regeneration by strontium-substituted calcium silicate bioactive ceramics. Biomaterials 2013;34(38):10028-42.

[125] Zhao F, Lei B, Li X, Mo Y, Wang R, Chen D, et al. Promoting in vivo early angiogenesis with sub-micrometer strontium-contained bioactive microspheres through modulating macrophage phenotypes. Biomaterials 2018;178:36-47.

[126] Walker J, Shadanbaz S, Woodfield TBF, Staiger MP, Dias GJ. Magnesium biomaterials for orthopedic application: a review from a biological perspective. J. Biomed. Mater. Res. Part B 2014;102(6):1316-31.

[127] Maier JAM, Bernardini D, Rayssiguier Y, Mazur A. High concentrations of magnesium modulate vascular endothelial cell behaviour in vitro. Biochim Biophys Acta-Mol Basis Dis 2004;1689(1):6-12.

[128] Maier JAM. Endothelial cells and magnesium: implications in atherosclerosis. Clin Sci 2012;122(9-10):397-407.

[129] Wang M, Yu Y, Dai K, Ma Z, Liu Y, Wang J, et al. Improved osteogenesis and angiogenesis of magnesium-doped calcium phosphate cement via macrophage immunomodulation. Biomater Sci 2016;4(11):1574-83.

[130] Dierichs L, Kloubert V, Rink L. Cellular zinc homeostasis modulates polarization of THP-1-derived macrophages. D - 100888704 2018;57(6):2161-69.

[131] Augustine R, Dominic EA, Reju I, Kaimal B, Kalarikkal N, Thomas S. Investigation of angiogenesis and its mechanism using zinc oxide nanoparticle-loaded electrospun tissue engineering scaffolds. RSC Adv 2014;4(93):51528-36.

[132] Barui AK, Nethi SK, Patra CR. Investigation of the role of nitric oxide driven angiogenesis by zinc oxide nanoflowers. J Mater Chem B 2017;5(18):3391-403.

[133] Seo HJ, Cho YE, Kim T, Shin HI, Kwun IS. Zinc may increase bone formation through stimulating cell proliferation, alkaline phosphatase activity and collagen synthesis in osteoblastic MC3T3-E1 cells. Nutr Res Pract 2010;4(5):356-61.

[134] Okazaki Y, Gotoh E, Manabe T, Kobayashi K. Comparison of metal concentrations in rat tibia tissues with various metallic implants. Biomaterials 2004;25(28):5913-20

[135] Cadosch D, Chan E, Gautschi OP, Meagher J, Zellweger R, Filgueira L. Titanium IV ions induced human osteoclast differentiation and enhanced bone resorption in vitro. J Biomed Mater Res Part A 2009;91A(1):29-36.

[136] Navarro M, Ginebra M-P, Clément J, Salvador M, Gloria A, Planell JA. Physicochemical degradation of titania-stabilized soluble phosphate glasses for medical applications. J Am Ceram Soc 2004;86(8):1345-52.

[137] Aguirre A, Gonzalez A, Navarro M, Castano O, Planell J, Engel E. Control of microenvironmental cues with a smart biomaterial composite promotes, D 100973416 2012;24:90-106.

[138] Chen S, Hou Y, Cheng G, Zhang C, Wang S, Zhang J. Cerium oxide nanoparticles protect endothelial cells from apoptosis induced by oxidative stress. Biol. Trace Elem. Res. 2013;154(1):156-66.

[139] Das S, Singh S, Dowding JM, Oommen S, Kumar A, Sayle TXT, et al. The induction of angiogenesis by cerium oxide nanoparticles through the modulation of oxygen in intracellular environments. Biomaterials 2012;33(31):7746-55.

[140] Xiang J, Li J, He J, Tang X, Dou C, Cao Z, et al. Cerium oxide nanoparticle modified scaffold interface enhances vascularization of bone grafts by activating calcium channel of mesenchymal stem cells. ACS Appl. Mater. Interfaces 2016:8(7):4489-99.

[141] Mahapatra C, Singh RK, Lee J-H, Jung J, Hyun JK, Kim H-W. Nano-shape varied cerium oxide nanomaterials rescue human dental stem cells from oxidative insult through intracellular or extracellular actions. Acta Biomater. 2017;50:142-53.

[142] Oike Y, Ito Y, Hamada K, Zhang X-Q, Miyata K, Arai F, et al. Regulation of vasculogenesis and angiogenesis by EphB/ephrin-B2 signaling between endothelial cells and surrounding mesenchymal cells. Blood 2002;100(4):1326.

[143] Jabbarzadeh E, Starnes T, Khan YM, Jiang T, Wirtel AJ, Deng M, et al. Induction of angiogenesis in tissue-engineered scaffolds designed for bone repair: a combined gene therapy-cell transplantation approach. Proc Natl Acad Sci 2008;105(32):11099-104.

[144] Hui Kian W, Chee Ren Ivan L, Feng W, Seow Khoon Mark C, Nguan Soon T, Chan J, Mintu P, Lay Poh T. Novel method to improve vascularization of tissue engineered constructs with biodegradable fibers. Biofabrication 2016;8(1):015004.

[145] Choi S-W, Zhang Y, MacEwan MR, Xia Y. Neovascularization in biodegradable inverse opal scaffolds with uniform and precisely controlled pore sizes. Adv Healthcare Mater 2013;2(1):145-54.

[146] Van Vlierberghe S, Dubruel P, Schacht E. Biopolymer-based hydrogels as scaffolds for tissue engineering applications: a review. Biomacromolecules 
2011;12(5):1387-408

[147] Chen Z, Klein T, Murray RZ, Crawford R, Chang J, Wu C, et al. Osteoimmunomodulation for the development of advanced bone biomaterials. Mater Today 2016;19(6):304-21.

[148] Ferrara N, Davis-Smyth T. The biology of vascular endothelial growth factor. Endocr Rev 1997;18(1):4-25.

[149] Roy H, Bhardwaj S, Ylä-Herttuala S. Biology of vascular endothelial growth factors. FEBS Lett 2006;580(12):2879-87.

[150] Luttun A, Tjwa M, Carmeliet P. Placental growth factor (PlGF) and its receptor Flt-1 (VEGFR-1). Ann N Y Acad Sci 2002;979(1):80-93.

[151] Tjwa M, Luttun A, Autiero M, Carmeliet P. VEGF and PlGF: two pleiotropic growth factors with distinct roles in development and homeostasis. Cell Tissue Res 2003;314(1):5-14.

[152] Simons M, Gordon E, Claesson-Welsh L. Mechanisms and regulation of endothelial VEGF receptor signalling. Nat Rev Mol Cell Biol Adv Online Publ 2016.

[153] Katagiri T, Takahashi N. Regulatory mechanisms of osteoblast and osteoclast differentiation. Oral Dis 2002;8(3):147-59.

[154] Pugh CW, Ratcliffe PJ. The von Hippel-Lindau tumor suppressor, hypoxia-inducible factor-1 (HIF-1) degradation, and cancer pathogenesis. Semin Cancer Biol 2003;13(1):83-9.

[155] Roy BSH, Babu M, Jauhiainen S, Herzig KH, Bellu AR, Haisma HJ, Carmeliet P, Alitalo K, Ylä-Herttuala S. Adenovirus-mediated gene transfer of placental growth factor to perivascular tissue induces angiogenesis via upregulation of the expression of endogenous vascular endothelial growth factor-A. Hum Gene Ther 2005;16(12):1422-8.

[156] Mould AW, Tonks ID, Cahill MM, Pettit AR, Thomas R, Hayward NK, et al. Vegfb gene knockout mice display reduced pathology and synovial angiogenesis in both antigen-induced and collagen-induced models of arthritis. Arthritis Rheum 2003;48(9):2660-9.

[157] Enholm B, Karpanen T, Jeltsch M, Kubo H, Stenback F, Prevo R, et al. Adenoviral expression of vascular endothelial growth factor-C induces lymphangiogenesis in the skin. Circ Res 2001;88(6):623-9.

[158] Jeltsch M, Kaipainen A, Joukov V, Meng X, Lakso M, Rauvala H, et al. Hyperplasia of lymphatic vessels in VEGF-C transgenic mice. Science 1997;276(5317):1423-5.

[159] Cao R, Ji H, Feng N, Zhang Y, Yang X, Andersson P, et al. Collaborative interplay between FGF-2 and VEGF-C promotes lymphangiogenesis and metastasis. Proc Natl Acad Sci 2012;109(39):15894-9.

[160] Orlandini M, Spreafico A, Bardelli M, Rocchigiani M, Salameh A, Nucciotti S, et al. Vascular endothelial growth factor-D activates VEGFR-3 expressed in osteoblasts inducing their differentiation. J Biol Chem 2006:281(26):17961-7.

[161] Kaigler D, Wang Z, Horger K, Mooney DJ, Krebsbach PH. VEGF scaffolds enhance angiogenesis and bone regeneration in irradiated osseous defects. J Bone Miner Res 2006;21(5):735-44.

[162] Kent Leach J, Kaigler D, Wang Z, Krebsbach PH, Mooney DJ. Coating of VEGF-releasing scaffolds with bioactive glass for angiogenesis and bone regeneration. Biomaterials 2006;27(17):3249-55.

[163] Ito Y. Covalently immobilized biosignal molecule materials for tissue engineering. Soft Matter 2008;4(1):46-56.

[164] Chen TT, Luque A, Lee S, Anderson SM, Segura T, Iruela-Arispe ML. Anchorage of VEGF to the extracellular matrix conveys differential signaling responses to endothelial cells. J Cell Biol 2010;188(4):595.

[165] Chiu LLY, Radisic M. Scaffolds with covalently immobilized VEGF and Angiopoietin-1 for vascularization of engineered tissues. Biomaterials 2010;31(2):226-41.

[166] D'Andrea LD, Iaccarino G, Fattorusso R, Sorriento D, Carannante C, Capasso D, et al. Targeting angiogenesis: Structural characterization and biological properties of a de novo engineered VEGF mimicking peptide. PNAS 2005;102(40):14215-20.

[167] Leslie-Barbick JE, Saik JE, Gould DJ, Dickinson ME, West JL. The promotion of microvasculature formation in poly(ethylene glycol) diacrylate hydrogels by an immobilized VEGF-mimetic peptide. Biomaterials 2011;32(25):5782-9.

[168] Chan TR, Stahl PJ, Yu SM. Matrix-bound VEGF mimetic peptides: design and endothelial-cell activation in collagen scaffolds. Adv Funct Mater $2011 ; 21: 4252-62$.

[169] Sato N, Beitz JG, Kato J, Yamamoto M, Clark JW, Calabresi P, et al. Platelet-derived growth factor indirectly stimulates angiogenesis in vitro. Am J Pathol 1993;142(4):1119-30.

[170] Kubota K, Sakikawa C, Katsumata M, Nakamura T, Wakabayashi K. Platelet-derived growth factor BB secreted from osteoclasts acts as an osteoblastogenesis inhibitory factor. J Bone Miner Res 2002;17(2):257-65.

[171] Li X, Tjwa M, Moons L, Fons P, Noel A, Ny A, et al. Revascularization of ischemic tissues by PDGF-CC via effects on endothelial cells and their progenitors. J Clin Investig 2005;115(1):118-27.

[172] Xie H, Cui Z, Wang L, Xia Z, Hu Y, Xian L, et al. PDGF-BB secreted by preosteoclasts induces angiogenesis during coupling with osteogenesis. Nat Med 2014;20(11):1270-8.

[173] Zhang Y, Ma Y, Wu C, Miron RJ, Cheng X. Platelet-derived growth factor BB gene-released scaffolds: biosynthesis and characterization. J Tissue Eng Regener Med 2016;10(10):E372-81.

[174] Ma C, Wei Q, Cao B, Cheng X, Tian J, Pu H, et al. A multifunctional bioactive material that stimulates osteogenesis and promotes the vascularization bone marrow stem cells and their resistance to bacterial infection. PLoS ONE 2017;12(3):e0172499.

[175] Saik JE, Gould DJ, Watkins EM, Dickinson ME, West JL. Covalently immobilized platelet-derived growth factor-BB promotes angiogenesis in biomimetic poly (ethylene glycol) hydrogels. Acta Biomater 2011;7(1):133-43.

[176] Ornitz DM, Itoh N. Fibroblast growth factors. Genome Biol 2001;2(3). reviews3005.1.

[177] Yun Y-R, Won JE, Jeon E, Lee S, Kang W, Jo H, et al. Fibroblast growth factors: biology, function, and application for tissue regeneration. J Tissue Eng 2010;1(1):218142

[178] Montesano R, Vassalli JD, Baird A, Guillemin R, Orci L. Basic fibroblast growth factor induces angiogenesis in vitro. Proc Natl Acad Sci 1986;83(19):7297-301.

[179] Seghezzi G, Patel S, Ren CJ, Gualandris A, Pintucci G, Robbins ES, et al. Fibroblast Growth Factor-2 (FGF-2) induces vascular endothelial growth factor (vegf) expression in the endothelial cells of forming capillaries: an autocrine mechanism contributing to angiogenesis. J Cell Biol 1998;141(7):1659-73.

[180] Fei Y, Gronowicz G, Hurley MM. Fibroblast growth factor-2, bone homeostasis and fracture repair. Curr Pharm Des 2013;19(19):3354-63.

[181] Schmid GJ, Kobayashi C, Sandell LJ, Ornitz DM. Fibroblast growth factor expression during skeletal fracture healing in mice. Dev Dyn 2009;238(3):776-1774.

[182] Du X, Xie Y, Xian CJ, Chen L. Role of FGFs/FGFRs in skeletal development and bone regeneration. J Cell Physiol 2012;227(12):3731-43.

[183] Kigami R, Sato S, Tsuchiya N, Sato N, Suzuki D, Arai Y, et al. Effect of basic fibroblast growth factor on angiogenesis and bone regeneration in non-critical-size bone defects in rat calvaria. J Oral Sci 2014;56(1):17-22.

[184] Yamaguchi K, Kaji Y, Nakamura O, Tobiume S, Yamamoto T. Prefabrication of vascularized allogenic bone graft in a rat by implanting a flow-through vascular pedicle and basic fibroblast growth factor containing hydroxyapatite/collagen composite. J Reconstr Microsurg 2017;33(05):367-76.

[185] Zhang H, Kot A, Lay Y-AE, Fierro FA, Chen H, Lane NE, et al. Acceleration of fracture healing by overexpression of basic fibroblast growth factor in the mesenchymal stromal cells. Stem Cells Transl Med 2017;6(10):1880-93.

[186] Chu H, Gao J, Chen C-W, Huard J, Wang Y. Injectable fibroblast growth factor-2 coacervate for persistant angiogenesis. Proc Natl Acad Sci USA 2011;108:13444-9.

[187] Moncion A, Lin M, O’Neill EG, Franceschi RT, Kripfgans OD, Putnam AJ, Fabiilli ML. Controlled release of basic fibroblast growth factor for angiogenesis using acoustically-responsive scaffolds. Biomaterials 2017;140(Supplement C):26-36.

[188] Attisano L, Wrana JL. Signal transduction by the TGF- $\beta$ superfamily. Science 2002;296(5573):1646.

[189] Shi Y, Massagué J. Mechanisms of TGF- $\beta$ signaling from cell membrane to the nucleus. Cell 2003;113(6):685-700.

[190] Madry H, Rey-Rico A, Venkatesan JK, Johnstone B, Cucchiarini M. Transforming growth factor beta-releasing scaffolds for cartilage tissue engineering. Tissue Eng Part B: Rev 2013;20(2):106-25.

[191] Linkhart TA, Mohan S, Baylink DJ. Growth factors for bone growth and repair: IGF, TGFß and BMP. Bone 1996;19(Supplement 1):S1-12.

[192] Zhao L, Jiang S, Hantash BM. Transforming growth factor B1 induces osteogenic differentiation of murine bone marrow stromal cells. Tissue Eng Part A 
2009;16(2):725-33.

[193] Guerrero PA, McCarty JH. TGF- $\beta$ activation and signaling in angiogenesis. IntechOpen; 2017.

[194] Yamamoto M, Tabata Y, Hong L, Miyamoto S, Hashimoto N, Ikada Y. Bone regeneration by transforming growth factor $\beta 1$ released from a biodegradable hydrogel. J Control Release 2000;64(1):133-42.

[195] Shakir S, MacIsaac ZM, Naran S, Smith DM, Bykowski MR, Cray JJ, et al. Transforming growth factor beta 1 augments calvarial defect healing and promotes suture regeneration. Tissue Eng Part A 2014;21(5-6):939-47.

[196] Möller-Siegert J, Parmentier J, Laquerrière P, Ouadi A, Raisslé O, Jallot E, et al. Physicochemical regulation of TGF and VEGF delivery from mesoporous calcium phosphate bone substitutes. Nanomedicine 2017;12(15):1835-50.

[197] Watarai A, Schirmer L, Thönes S, Freudenberg U, Werner C, Simon JC, et al. TGFß functionalized starPEG-heparin hydrogels modulate human dermal fibroblast growth and differentiation. Acta Biomater 2015;25:65-75.

[198] Zhang J, Dalbay MT, Luo X, Vrij E, Barbieri D, Moroni L, et al. Topography of calcium phosphate ceramics regulates primary cilia length and TGF receptor recruitment associated with osteogenesis. Acta Biomater 2017;57:487-97.

[199] Bai Y, Bai L, Zhou J, Chen H, Zhang L. Sequential delivery of VEGF, FGF-2 and PDGF from the polymeric system enhance HUVECs angiogenesis in vitro and CAM angiogenesis. Cell Immunol 2017.

[200] Bayer EA, Jordan J, Roy A, Gottardi R, Fedorchak MV, Kumta PN, et al. Programmed platelet-derived growth factor-BB and bone morphogenetic protein-2 delivery from a hybrid calcium phosphate/alginate scaffold. Tissue Eng Part A 2017.

[201] Kang MS, Kim J-H, Singh RK, Jang J-H, Kim H-W. Therapeutic-designed electrospun bone scaffolds: Mesoporous bioactive nanocarriers in hollow fiber composites to sequentially deliver dual growth factors. Acta Biomater 2015;16:103-16.

[202] Subbiah R, Hwang MP, Van SY, Do SH, Park H, Lee K, et al. Osteogenic/angiogenic dual growth factor delivery microcapsules for regeneration of vascularized bone tissue. Adv Healthcare Mater 2015;4(13):1982-92.

[203] Patel ZS, Young S, Tabata Y, Jansen JA, Wong MEK, Mikos AG. Dual delivery of an angiogenic and an osteogenic growth factor for bone regeneration in a critical size defect model. Bone 2008;43(5):931-40.

[204] Bao X, Zhu L, Huang X, Tang D, He D, Shi J, et al. 3D biomimetic artificial bone scaffolds with dual-cytokines spatiotemporal delivery for large weight-bearing bone defect repair. Sci Rep 2017;7(1):7814.

[205] Barati D, Shariati SRP, Moeinzadeh S, Melero-Martin JM, Khademhosseini A, Jabbari E. Spatiotemporal release of BMP-2 and VEGF enhances osteogenic and vasculogenic differentiation of human mesenchymal stem cells and endothelial colony-forming cells co-encapsulated in a patterned hydrogel. J Controlled Release 2016;223(Supplement C):126-36.

[206] Perez RA, Kim JH, Buitrago JO, Wall IB, Kim HW. Novel therapeutic core-shell hydrogel scaffolds with sequential delivery of cobalt and bone morphogenetic protein-2 for synergistic bone regeneration. Acta Biomater 2015;23:295-308.

[207] Curtin CM, Tierney EG, McSorley K, Cryan S-A, Duffy GP, O'Brien FJ. Combinatorial gene therapy accelerates bone regeneration: non-viral dual delivery of VEGF and BMP2 in a collagen-nanohydroxyapatite scaffold. Adv Healthcare Mater 2015;4:223-7.

[208] Raftery RM, Mencía Castaño I, Chen G, Cavanagh B, Quinn B, Curtin CM, et al. Translating the role of osteogenic-angiogenic coupling in bone formation: highly efficient chitosan-pDNA activated scaffolds can accelerate bone regeneration in critical-sized bone defects. Biomaterials 2017;149:116-27.

[209] Briquez PS, Clegg LE, Martino MM, Gabhann FM, Hubbell JA. Design principles for therapeutic angiogenic materials. Nat Rev Mater 2016;1:15006.

[210] Martino MM, Briquez PS, Güç E, Tortelli F, Kilarski WW, Metzger S, et al. Growth factors engineered for super-affinity to the extracellular matrix enhance tissue healing. Science 2014;343(6173):885.

[211] Nih LR, Gojgini S, Carmichael ST, Segura T. Dual-function injectable angiogenic biomaterial for the repair of brain tissue following stroke. Nat Mater 2018;17(7):642-51.

[212] Fairbrother WJ, Champe MA, Christinger HW, Keyt BA, Starovasnik MA. Solution structure of the heparin-binding domain of vascular endothelial growth factor. Structure 1998;6(5):637-48.

[213] Ishihara J, Ishihara A, Fukunaga K, Sasaki K, White MJV, Briquez PS, et al. Laminin heparin-binding peptides bind to several growth factors and enhance diabetic wound healing. Nat Commun 2018;9(1):2163.

[214] Schwartz MA. Integrins and Extracellular Matrix in Mechanotransduction. Cold Spring Harbor Perspect Biol 2010;2(12).

[215] Strömblad S, Cheresh DA. Integrins, angiogenesis and vascular cell survival. Chem Biol 1996;3(11):881-5.

[216] Abraham S, Kogata N, Fässler R, Adams RH. Integrin $\beta 1$ subunit controls mural cell adhesion, spreading, and blood vessel wall stability. Circ Res 2008.

[217] Shen Y, Gao M, Ma Y, Yu H, Cui F-Z, Gregersen H, et al. Effect of surface chemistry on the integrin induced pathway in regulating vascular endothelial cells migration. Colloids Surf, B 2015;126:188-97.

[218] Lei Y, Zouani OF, Remy M, Ayela C, Durrieu M-C. Geometrical microfeature cues for directing tubulogenesis of endothelial cells. PLoS ONE 2012;7(7):e41163.

[219] Li S, Nih LR, Bachman H, Fei P, Li Y, Nam E, et al. Hydrogels with precisely controlled integrin activation dictate vascular patterning and permeability. Nat Mater 2017;16(9):953-61.

[220] Mahabeleshwar GH, Feng W, Reddy K, Plow EF, Byzova TV. Mechanisms of integrin-vascular endothelial growth factor receptor cross-activation in angiogenesis. Circ Res 2007;101(6):570.

[221] García JR, Clark AY, García AJ. Integrin-specific hydrogels functionalized with VEGF for vascularization and bone regeneration of critical-size bone defects. J Biomed Mater Res Part A 2016;104(4):889-900.

[222] Leiss M, Beckmann K, Girós A, Costell M, Fässler R. The role of integrin binding sites in fibronectin matrix assembly in vivo. Curr Opin Cell Biol 2008;20(5):502-7.

[223] Karp JM, Leng Teo GS. Mesenchymal stem cell homing: the devil is in the details. Cell Stem Cell 2009;4(3):206-16.

[224] W Z, C Z, Y W, D Y, S W, D Z, et al. VEGF and BMP-2 promote bone regeneration by facilitating bone marrow stem cell homing and differentiation. Eur Cell Mater 2014;27:1-12.

[225] Street J, Bao M, deGuzman L, Bunting S, Peale FV, Ferrara N, et al. Vascular endothelial growth factor stimulates bone repair by promoting angiogenesis and bone turnover. Proc Natl Acad Sci 2002;99(15):9656.

[226] Bragdon B, Moseychuk O, Saldanha S, King D, Julian J, Nohe A. Bone morphogenetic proteins: a critical review. Cell Signal 2011;23(4):609-20.

[227] García de Vinuesa A, Abdelilah-Seyfried S, Knaus P, Zwijsen A, Bailly S. BMP signaling in vascular biology and dysfunction. Cytokine Growth Factor Rev 2016;27:65-79.

[228] Benn A, Hiepen C, Osterland M, Schütte C, Zwijsen A, Knaus P. Role of bone morphogenetic proteins in sprouting angiogenesis: differential BMP receptordependent signaling pathways balance stalk vs. tip cell competence. FASEB J 2017;31(11):4720-33.

[229] Kirkpatrick CJ, Fuchs S, Unger RE. Co-culture systems for vascularization-learning from nature. Adv Drug Deliv Rev 2011;63(4-5):291-9.

[230] Fuchs S, Hofmann A, Kirkpatrick C. Microvessel-like structures from outgrowth endothelial cells from human peripheral blood in 2-dimensional and 3dimensional co-cultures with osteoblastic lineage cells. Tissue Eng 2007;13(10):2577-88.

[231] Gershovich JG, Dahlin RL, Kasper FK, Mikos AG. Enhanced osteogenesis in cocultures with human mesenchymal stem cells and endothelial cells on polymeric microfiber scaffolds. Tissue Eng Part A 2013;19(23-24):2565-76.

[232] Steiner D, Lampert F, Stark GB, Finkenzeller G. Effects of endothelial cells on proliferation and survival of human mesenchymal stem cells and primary osteoblasts. J Orthop Res 2012;30(10):1682-9.

[233] Tansriratanawong K, Tamaki Y, Ishikawa H, Sato S. Co-culture with periodontal ligament stem cells enhances osteogenic gene expression in de-differentiated fat cells. Hum Cell 2014;27(4):151-61.

[234] DR K, TK H, H S, BJ H, Z W, MJ B. Oxygen tension regulates the expression of angiogenesis factor by macrophages. Science 1983;221(4617):1283-85.

[235] Bosenberg MW, Massagué J. Juxtacrine cell signaling molecules. Curr Opin Cell Biol 1993;5(5):832-8.

[236] Villars F, Bordenave L, Bareille R, Amédée J. Effect of human endothelial cells on human bone marrow stromal cell phenotype: role of VEGF? J Cell Biochem 2000;79(4):672-85. 
[237] Herzog DPE, Dohle E, Bischoff I, Kirkpatrick CJ. Cell communication in a coculture system consisting of outgrowth endothelial cells and primary osteoblasts. Biomed Res Int 2014;2014(320123):15.

[238] Bendinelli P, Maroni P, Matteucci E, Desiderio M. Cell and signal components of the microenvironment of bone metastasis are affected by hypoxia. Int J Mol Sci 2016;17(5):706.

[239] Dunlop LL, Hall BK. Relationships between cellular condensation, preosteoblast formation and epithelial-mesenchymal interactions in initiation of osteogenesis. Int J Dev Biol 1995;39:357-71.

[240] Dohle E, Bischoff I, Böse T, Marsano A, Banfi A, Unger RE, et al. Macrophage-mediated angiogenic activation of outgrowth endothelial cells in co-culture with primary osteoblasts. Eur Cell Mater 2014:149-64.

[241] Herath Thanuja DK, Larbi A, Teoh Swee H, Kirkpatrick CJ, Goh Bee T. Neutrophil-mediated enhancement of angiogenesis and osteogenesis in a novel triple cell co-culture model with endothelial cells and osteoblasts. J Tissue Eng Regener Med 2018;12(2):e1221-36.

[242] Tazzyman S, Lewis Claire E, Murdoch C. Neutrophils: key mediators of tumour angiogenesis. Int J Exp Pathol 2009;90(3):222-31.

[243] Gong Y, Koh D-R. Neutrophils promote inflammatory angiogenesis via release of preformed VEGF in an in vivo corneal model. Cell Tissue Res 2010;339(2):437-48.

[244] Olfert IM, Baum O, Hellsten Y, Egginton S. Advances and challenges in skeletal muscle angiogenesis. Am J Physiol-Heart Circul Physiol 2015;310(3):H326-36.

[245] Badylak SF, Dziki JL, Sicari BM, Ambrosio F, Boninger ML. Mechanisms by which acellular biologic scaffolds promote functional skeletal muscle restoration. Biomaterials 2016;103:128-36.

[246] Birbrair A, Zhang T, Wang Z-M, Messi ML, Mintz A, Delbono O. Pericytes: multitasking cells in the regeneration of injured, diseased, and aged skeletal muscle. Front Aging Neurosci 2014;6(245).

[247] Juhas M, Abutaleb N, Wang JT, Ye J, Shaikh Z, Sriworarat C, et al. Incorporation of macrophages into engineered skeletal muscle enables enhanced muscle regeneration. Nat Biomed Eng 2018;2(12):942-54.

[248] Latroche C, Weiss-Gayet M, Muller L, Gitiaux C, Leblanc P, Liot S, et al. Coupling between myogenesis and angiogenesis during skeletal muscle regeneration is stimulated by restorative macrophages. Stem Cell Rep 2017;9(6):2018-33.

[249] Germani A, Di Carlo A, Mangoni A, Straino S, Giacinti C, Turrini P, et al. Vascular endothelial growth factor modulates skeletal myoblast function. Am J Pathol 2003;163(4):1417-28.

[250] Wang HD, Guo Q, Quan A, Lopez J, Alonso-Escalante JC, Lough DM, et al. Vascular endothelial growth factor induction of muscle-derived stem cells enhances vascular phenotype while preserving myogenic potential. Ann Plast Surg 2017;79(4):404-9.

[251] Deasy BM, Feduska JM, Payne TR, Li Y, Ambrosio F, Huard J. Effect of VEGF on the regenerative capacity of muscle stem cells in dystrophic skeletal muscle. Mol Therapy: J Am Soc Gene Therapy 2009;17(10):1788-98.

[252] Koffler J, Kaufman-Francis K, Shandalov Y, Egozi D, Pavlov DA, Landesberg A, et al. Improved vascular organization enhances functional integration of engineered skeletal muscle grafts. Proc Natl Acad Sci 2011;108(36):14789-94.

[253] Tidball JG, Dorshkind K, Wehling-Henricks M. Shared signaling systems in myeloid cell-mediated muscle regeneration. Development 2014;141(6):1184-96.

[254] Ohab JJ, Fleming S, Blesch A, Carmichael ST. A neurovascular niche for neurogenesis after stroke. J Neurosci 2006;26(50):13007-16.

[255] Kundi S, Bicknell R, Ahmed Z. The role of angiogenic and wound-healing factors after spinal cord injury in mammals. Neurosci Res 2013;76(1-2):1-9.

[256] Kim J-W, Mahapatra C, Hong J-Y, Kim MS, Leong KW, Kim H-W, et al. Functional recovery of contused spinal cord in rat with the injection of optimal-dosed cerium oxide nanoparticles. Adv Sci 2017;4(10):1700034.

[257] De Laporte L, des Rieux A, Tuinstra HM, Zelivyanskaya ML, De Clerck NM, Postnov AA, et al. Vascular endothelial growth factor and fibroblast growth factor 2 delivery from spinal cord bridges to enhance angiogenesis following injury. J Biomed Mater Res A 2011;98(3):372-82.

[258] Rocha LA, Sousa RA, Learmonth DA, Salgado AJ. The role of biomaterials as angiogenic modulators of spinal cord injury: mimetics of the spinal cord, cell and angiogenic factor delivery agents. Front Pharmacol 2018;9:164.

[259] Mitrousis N, Fokina A, Shoichet MS. Biomaterials for cell transplantation. Nat Rev Mater 2018;3(11):441-56.

[260] Gurtner GC, Werner S, Barrandon Y, Longaker MT. Wound repair and regeneration. Nature 2008;453:314.

[261] Kang Y, Kim S, Fahrenholtz M, Khademhosseini A, Yang Y. Osteogenic and angiogenic potentials of monocultured and co-cultured human-bone-marrowderived mesenchymal stem cells and human-umbilical-vein endothelial cells on three-dimensional porous beta-tricalcium phosphate scaffold. Acta Biomater 2013;9(1):4906-15.

[262] Grellier M, Bordenave LJ. Amédée Cell-to-cell communication between osteogenic and endothelial lineages: implications for tissue engineering. Trends Biotechnol 2009;27(10):562-71.

[263] Kaigler D, Krebsbach PH, West ER, Horger K, Huang Y-C, Mooney DJ. Endothelial cell modulation of bone marrow stromal cell osteogenic potential. FASEB J 2005.

[264] Santos MI, Tuzlakoglu K, Fuchs S, Gomes ME, Peters K, Unger RE, et al. Endothelial cell colonization and angiogenic potential of combined nano- and microfibrous scaffolds for bone tissue engineering. Biomaterials 2008;29(32):4306-13.

[265] Tibbitt Mark W, Anseth Kristi S. Hydrogels as extracellular matrix mimics for 3D cell culture. Biotechnol Bioeng 2009;103(4):655-63.

[266] Wenger A, Stahl A, Weber H, Finkenzeller G, Augustin HG, Stark GB, et al. Modulation of in vitro angiogenesis in a three-dimensional spheroidal coculture model for bone tissue engineering. Tissue Eng 2004;10(9-10):1536-47.

[267] Stahl A, Wu X, Wenger A, Klagsbrun M, Kurschat P. Endothelial progenitor cell sprouting in spheroid cultures is resistant to inhibition by osteoblasts: a model for bone replacement grafts. FEBS Lett 2005;579(24):5338-42.

[268] Xing Q, Qian Z, Kannan B, Tahtinen M, Zhao F. Osteogenic differentiation evaluation of an engineered extracellular matrix based tissue sheet for potential periosteum replacement. ACS Appl Mater Interfaces 2015;7(41):23239-47.

[269] Hu K, Olsen BR. Osteoblast-derived VEGF regulates osteoblast differentiation and bone formation during bone repair. J Clin Investig 2016;126(2):509-26.

[270] Clarkin CE, Emery RJ, Pitsillides AA, Wheeler-Jones CPD. Evaluation of VEGF-mediated signaling in primary human cells reveals a paracrine action for VEGF in osteoblast-mediated crosstalk to endothelial cells. J Cell Physiol 2008;214(2):537-44.

[271] Aguirre A, Planell JA, Engel E. Dynamics of bone marrow-derived endothelial progenitor cell/mesenchymal stem cell interaction in co-culture and its implications in angiogenesis. Biochem Biophys Res Commun 2010;400(2):284-91.

[272] Chen L, Tredget EE, Wu PYG, Wu Y. Paracrine factors of mesenchymal stem cells recruit macrophages and endothelial lineage cells and enhance wound healing. PLoS ONE 2008;3(4):e1886.

[273] Grellier M, Granja PL, Fricain J-C, Bidarra SJ, Renard M, Bareille R, et al. The effect of the co-immobilization of human osteoprogenitors and endothelial cells within alginate microspheres on mineralization in a bone defect. Biomaterials 2009;30(19):3271-8.

[274] Hsu S-H, Ho T-T, Huang N-C, Yao C-L, Peng L-H, Dai N-T. Substrate-dependent modulation of 3D spheroid morphology self-assembled in mesenchymal stem cell-endothelial progenitor cell coculture. Biomaterials 2014;35(26):7295-307.

[275] Wei S, Antonella M, Yang S, Andreas S, Harald S, Stanislav NG, et al. Co-culture of outgrowth endothelial cells with human mesenchymal stem cells in silk fibroin hydrogels promotes angiogenesis. Biomed Mater 2016;11(3):035009.

[276] Chwalek K, Tsurkan MV, Freudenberg U, Werner C. Glycosaminoglycan-based hydrogels to modulate heterocellular communication in in vitro angiogenesis models. Sci Rep 2014;4:4414.

[277] Kyriakidou K, Lucarini G, Zizzi A, Salvolini E, Mattioli Belmonte M, Mollica F, et al. Dynamic co-seeding of osteoblast and endothelial cells on 3D polycaprolactone scaffolds for enhanced bone tissue engineering. J Bioactive Compatible Polym 2008;23(3):227-43.

[278] Barron MJ, Goldman J, Tsai C-J, Donahue SW. Perfusion flow enhances osteogenic gene expression and the infiltration of osteoblasts and endothelial cells into three-dimensional calcium phosphate scaffolds. Int J Biomater 2012;2012(915620):10.

[279] Scherberich A, Galli R, Jaquiery C, Farhadi J, Martin I. Three-dimensional perfusion culture of human adipose tissue-derived endothelial and osteoblastic progenitors generates osteogenic constructs with intrinsic vascularization capacity. Stem Cells 2007;25(7):1823-9.

[280] Saleh FA, Whyte M, Genever PG. Effects of endothelial cells on human mesenchymal stem cell activity in a three-dimensional in vitro model. Eur Cell Mater 
2011;22:242-57. discussion 257.

[281] Park YK, Tu T-Y, Lim SH, Clement IJM, Yang SY, Kamm RD. In vitro microvessel growth and remodeling within a three-dimensional microfluidic environment. Cell Mol Bioeng 2014;7(1):15-25.

[282] Uwamori H, Higuchi T, Arai K, Sudo R. Integration of neurogenesis and angiogenesis models for constructing a neurovascular tissue. Sci Rep 2017;7(1):17349.

[283] Osaki T, Sivathanu V, Kamm RD. Engineered 3D vascular and neuronal networks in a microfluidic platform. Sci Rep 2018;8(1):5168.

[284] Vandenburgh H, Shansky J, Benesch-Lee F, Barbata V, Reid J, Thorrez L, et al. Drug-screening platform based on the contractility of tissue-engineered muscle. Muscle Nerve 2008;37(4):438-47.

[285] Dacha G, Lieselot D, Vicky VM, Linda D, Melanie G, Gilles C, et al. Endothelial network formation within human tissue-engineered skeletal muscle. Tissue Eng Part A 2015;21(19-20):2548-58.

[286] Shandalov Y, Egozi D, Koffler J, Dado-Rosenfeld D, Ben-Shimol D, Freiman A, et al. An engineered muscle flap for reconstruction of large soft tissue defects. Proc Natl Acad Sci 2014;111(16):6010-5.

[287] Ko IK, Lee B-K, Lee SJ, Andersson K-E, Atala A, Yoo JJ. The effect of in vitro formation of acetylcholine receptor (AChR) clusters in engineered muscle fibers on subsequent innervation of constructs in vivo. Biomaterials 2013;34(13):3246-55.

[288] Xu T, Zhao W, Zhu J-M, Albanna MZ, Yoo JJ, Atala A. Complex heterogeneous tissue constructs containing multiple cell types prepared by inkjet printing technology. Biomaterials 2013;34(1):130-9.

[289] Kwee BJ, Mooney DJ. Biomaterials for skeletal muscle tissue engineering. Curr Opin Biotechnol 2017;47:16-22.

[290] Lovett M, Lee K, Edwards A, Kaplan DL. Vascularization strategies for tissue engineering. Tissue Eng Part B: Rev 2009;15(3):353-70.

[291] Nguyen BN, Ko H, Fisher JP. Tunable osteogenic differentiation of hMPCs in tubular perfusion system bioreactor. Biotechnol Bioeng 2016;113(8):1805-13.

[292] Nishi M, Matsumoto R, Dong J, Uemura T. Engineered bone tissue associated with vascularization utilizing a rotating wall vessel bioreactor. J Biomed Mater Res Part A 2013;101A(2):421-7.

[293] Ma D, Ren L, Cao Z, Li J, Cao J, Tian W, et al. Prefabrication of axially vascularized bone by combining $\beta$-tricalciumphosphate, arteriovenous loop, and cell sheet technique. Tissue Eng Regen Med 2016;13(5):579-84.

[294] Liu H, Zhou W, Ren N, Feng Z, Dong Y, Bai S, et al. Cell sheets of co-cultured endothelial progenitor cells and mesenchymal stromal cells promote osseointegration in irradiated rat bone. Sci Rep 2017;7(1):3038.

[295] Bauman E, Feijão T, Carvalho DTO, Granja PL, Barrias CC. Xeno-free pre-vascularized spheroids for therapeutic applications. Sci Rep 2018;8(1):230.

[296] Bose S, Vahabzadeh S, Bandyopadhyay A. Bone tissue engineering using 3D printing. Mater Today 2013;16(12):496-504.

[297] Haberstroh K, Ritter K, Kuschnierz J, Bormann K-H, Kaps C, Carvalho C, et al. Bone repair by cell-seeded 3D-bioplotted composite scaffolds made of collagen treated tricalciumphosphate or tricalciumphosphate-chitosan-collagen hydrogel or PLGA in ovine critical-sized calvarial defects. J Biomed Mater Res B 2010;93B(2):530.

[298] Atala A, Yoo JJ. Essentials of 3D biofabrication and translation. Boston: Academic Press; 2015.

[299] Rhee S, Puetzer JL, Mason BN, Reinhart-King CA, Bonassar LJ. 3D bioprinting of spatially heterogeneous collagen constructs for cartilage tissue engineering. ACS Biomater Sci Eng 2016;2(10):1800-5.

[300] Ouyang L, Highley CB, Rodell CB, Sun W, Burdick JA. 3D printing of shear-thinning hyaluronic acid hydrogels with secondary cross-linking. ACS Biomater Sci Eng 2016;2(10):1743-51.

[301] Rodriguez MJ, Brown J, Giordano J, Lin SJ, Omenetto FG, Kaplan DL. Silk based bioinks for soft tissue reconstruction using 3-dimensional (3D) printing with in vitro and in vivo assessments. Biomaterials 2017;117:105-15.

[302] Pati F, Cho D-W. Bioprinting of 3D tissue models using decellularized extracellular matrix bioink. In: Koledova Z, editor. 3D cell culture: methods and protocols. New York, New York, NY: Springer; 2017. p. 381-90.

[303] Chimene D, Lennox KK, Kaunas RR, Gaharwar AK. Advanced bioinks for 3D printing: a materials science perspective. Ann. Biomed. Eng. 2016;44(6):2090-102.

[304] Faramarzi N, Yazdi IK, Nabavinia M, Gemma A, Fanelli A, Caizzone A, et al. Patient-specific bioinks for 3D bioprinting of tissue engineering scaffolds. Adv Healthcare Mater 2018;7(11):9.

[305] Gungor-Ozkerim PS, Inci I, Zhang YS, Khademhosseini A, Dokmeci MR. Bioinks for 3D bioprinting: an overview. Biomater Sci 2018;6(5):915-46.

[306] Mirabella T, MacArthur JW, Cheng D, Ozaki CK, Woo YJ, Yang MT, et al. 3D-printed vascular networks direct therapeutic angiogenesis in ischaemia. Nat Biomed Eng 2017;1:0083.

[307] Kim BS, Kwon YW, Kong J-S, Park GT, Gao G, Han W, et al. 3D cell printing of in vitro stabilized skin model and in vivo pre-vascularized skin patch using tissuespecific extracellular matrix bioink: a step towards advanced skin tissue engineering. Biomaterials 2018;168:38-53.

[308] Cui H, Zhu W, Holmes B, Zhang LG. Biologically inspired smart release system based on 3D bioprinted perfused scaffold for vascularized tissue regeneration. Adv Sci 2016;3(8):1600058.

[309] Zhai X, Ruan C, Ma Y, Cheng D, Wu M, Liu W, et al. 3D-bioprinted osteoblast-laden nanocomposite hydrogel constructs with induced microenvironments promote cell viability, differentiation, and osteogenesis both in vitro and in vivo. Adv Sci 2017;5(3):1700550.

[310] Kronenberg HM. Developmental regulation of the growth plate. Nature 2003;423:332.

[311] Scotti C, Piccinini E, Takizawa H, Todorov A, Bourgine P, Papadimitropoulos A, et al. Engineering of a functional bone organ through endochondral ossification. Proc Natl Acad Sci 2013;110(10):3997.

[312] Daly AC, Pitacco P, Nulty J, Cunniffe GM, Kelly DJ. 3D printed microchannel networks to direct vascularisation during endochondral bone repair. Biomaterials 2018;162:34-46.

[313] Jia W, Gungor-Ozkerim PS, Zhang YS, Yue K, Zhu K, Liu W, et al. Direct 3D bioprinting of perfusable vascular constructs using a blend bioink. Biomaterials 2016;106:58-68.

[314] Byambaa B, Annabi N, Yue K, Trujillo-de Santiago G, Alvarez MM, Jia W, et al. Bioprinted osteogenic and vasculogenic patterns for engineering 3D bone tissue. Adv Healthcare Mater 2017;6(16):1700015.

[315] Gao B, Yang Q, Zhao X, Jin G, Ma Y, Xu F. 4D bioprinting for biomedical applications. Trends Biotechnol 2016;34(9):746-56. 